\title{
Fundamental Study of Nano-Scale Adhesion and Friction Properties of Graphene in Ambient Air and Liquid Environments
}

Sai Suvineeth Ramayanam

Follow this and additional works at: https://researchrepository.wvu.edu/etd

\section{Recommended Citation}

Ramayanam, Sai Suvineeth, "Fundamental Study of Nano-Scale Adhesion and Friction Properties of Graphene in Ambient Air and Liquid Environments" (2015). Graduate Theses, Dissertations, and Problem Reports. 6470.

https://researchrepository.wvu.edu/etd/6470

This Thesis is protected by copyright and/or related rights. It has been brought to you by the The Research Repository @ WVU with permission from the rights-holder(s). You are free to use this Thesis in any way that is permitted by the copyright and related rights legislation that applies to your use. For other uses you must obtain permission from the rights-holder(s) directly, unless additional rights are indicated by a Creative Commons license in the record and/ or on the work itself. This Thesis has been accepted for inclusion in WVU Graduate Theses, Dissertations, and Problem Reports collection by an authorized administrator of The Research Repository @ WVU. For more information, please contact researchrepository@mail.wvu.edu. 


\title{
Fundamental Study of Nano-Scale Adhesion and Friction Properties of Graphene in Ambient Air and Liquid Environments
}

\section{Sai Suvineeth Ramayanam}

\author{
Thesis submitted to the \\ Benjamin M. Statler College of Engineering and Mineral Resources \\ At West Virginia University \\ In partial fulfillment of the requirements for the degree of

\section{Master of Science in Mechanical Engineering} \\ Approved Thesis Committee \\ Dr. Konstantinos Sierros, Committee Chair, Mechanical and Aerospace Engineering \\ Dr. Charter Stinespring, Chemical Engineering \\ Dr. Stephen Kukureka, Mechanical and Aerospace Engineering
}

Department of Mechanical and Aerospace Engineering

Morgantown, West Virginia, 2015

Keywords: Graphene - Adhesion - Friction - Atomic force Microscopy

Copyright 2015 Sai Suvineeth Ramayanam 


\section{ABSTRACT \\ Fundamental Study of Nano-Scale Adhesion and Friction Properties of Graphene in Ambient Air and Liquid Environments \\ Sai Suvineeth Ramayanam}

The aim of this study is to understand the fundamental tribological interactions of model contacts developed between a 'single' asperity silicon tip and a few layer graphene surface in ambient air, ionic liquid, and lubricating oil environments. The motivation to investigate such fundamental interactions stems from the need to gain an understanding of the tribological properties, morphology and defects of few layer graphene with respect to different synthesis methods including both bottom-up and top-down approaches. In particular, the surface properties of atomically thin sheets of graphene synthesized by three methods; (i) liquid phase exfoliation of graphene, (ii) chemical reduction of exfoliated graphene oxide, on a silicon oxide substrate, and (iii) graphene synthesis by halogen based plasma etching on a silicon carbide substrate are studied using atomic force microscopy, lateral force microscopy and x-ray photoelectron spectroscopy. Friction of Si 'single’ asperities sliding against a few layer graphene surface in ambient air, ionic liquid, and lubricating oil environments is reported. It is found that oxygen based defects play a major role in controlling the friction and adhesion properties of few layer graphene surfaces. The role of substrate and its bonding with the few layer graphene is also an important parameter. In liquids, we report a newly observed Stribeck like behavior in the nanoscale. This work can lead to important device applications with reduced friction such as contact-based microelectromechanical systems. It also sheds light on liquid-graphene interfacial characteristics which can be proved vital in applications spanning from electrochemical energy devices to nanolubricants. 


\section{ACKNOWLEDGEMENTS}

I wish to express my sincere gratitude towards Dr. Kostas Sierros for agreeing to be my research advisor and for his relentless support in guiding me through the trials and tribulations of graduate research. His extensive knowledge in the fields of material science and tribology was of utmost help throughout my research. His knowledge, enthusiasm and pursuit for excellence have inspired me beyond measure. I am extremely grateful to him for providing me with the opportunity to work with him.

My sincere appreciation and respect goes to Dr. Charter Stinespring, who provided me with the inputs on the topic of research and invaluable guidance and supervision throughout my research work. I thank him for letting me use his laboratory facilities and for other technical aspects related to my work.

I thank Dr. Stephen Kukureka for being part of the committee and providing inputs with his expertise on the subject.

I thank Nick Morris, Saurabh Chaudhari, Dr. Aaron Kessman and Sean Cronin for their invaluable contributions to this thesis study. I acknowledge the support of my labmates Derrick Banerjee, Maria Torres, Deji Abidakun, Mark Shoukry, Jacob Cordonier and all the undergraduates of the FEST group for their contributions and camaraderie.

Finally and most importantly, I express my sincere gratitude to my parents Narayana Murthy Ramayanam, Padmaja Ramayanam and my sister Samanvitha Ramayanam for their unconditional love, support, motivation and belief in me without which this thesis would not have been possible. 


\section{TABLE OF CONTENTS}

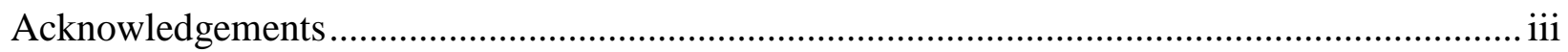

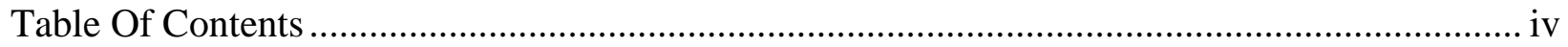

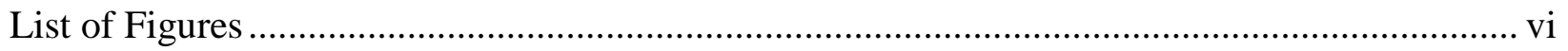

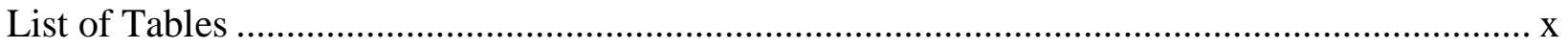

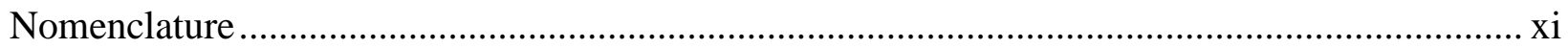

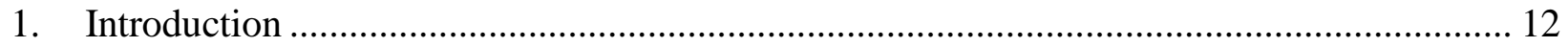

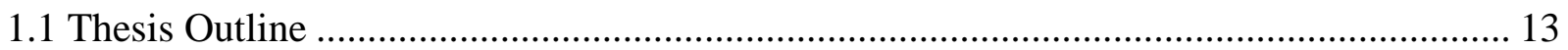

2. Graphene Synthesis and Tribological Characterization ........................................................ 15

2.1 Structure, Properties and Applications................................................................................ 15

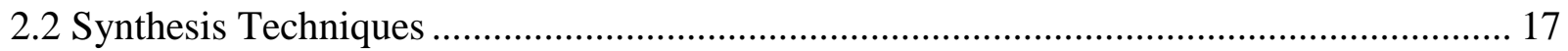

2.3 Tribological Characterization:................................................................................. 19

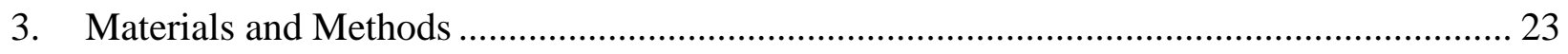

3.1 Substrate Preparation.................................................................................................... 23

3.2 Graphene Synthesis ........................................................................................................ 25

3.3 Material Characterization.............................................................................................. 29

3.3.1 Optical Microscopy ……………………………...................................................... 29

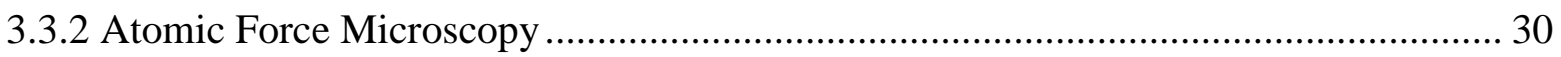

3.3.3 X-Ray Photoelectron Spectroscopy …………….......................................................... 37 


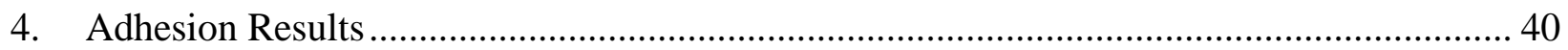

4.1 Edge-Centre Distance Effect on Adhesion.............................................................. 51

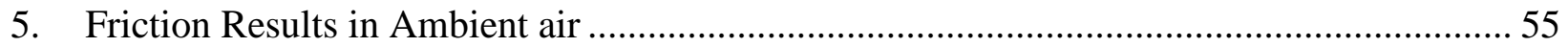

5.1 Study of friction in different domains of an exfoliated graphene flake .......................... 61

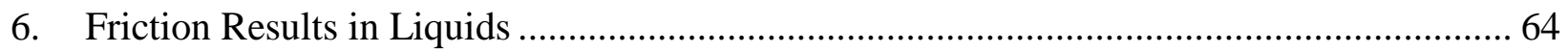

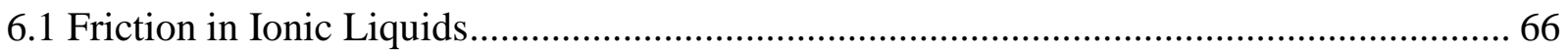

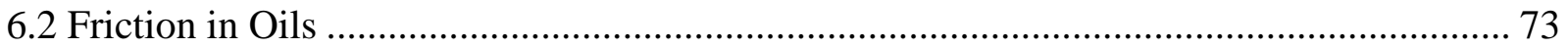

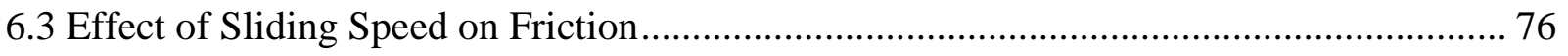

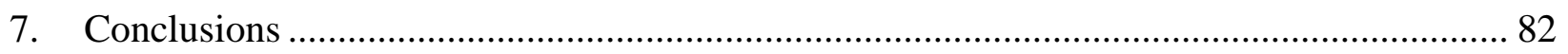

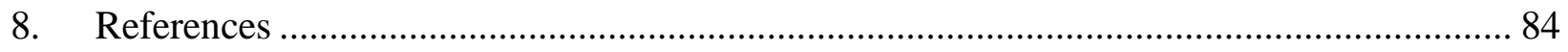




\section{LIST OF FIGURES}

Figure 1 2D graphene, 0D buckyballs, 1D nanotubes and 3D graphite [2]............................. 15

Figure 2 Relative Friction of flg (solid lines) and silicon dioxide (dashed lines) in air (red), water

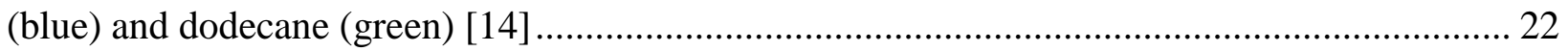

Figure 3 Optical image of prepared $\mathrm{SiO}_{2}$ substrate with gold numerical markers ..................... 24

Figure 4 Schematic depicting the photolithography process ............................................. 25

Figure 5 Schematic showing the preparation of flg flakes using liquid phase exfoliation technique

Figure 6 Schematic showing the preparation of reduced graphene oxide flakes....................... 28

Figure 7 Schematic showing synthesis of graphene using halogen based etching on SiC [48] ... 29

Figure 8 Optical image of a graphene flake $100 \mathrm{X}$ objective .............................................. 30

Figure 9 Schematic of an AFM operation (not to scale) ......................................................... 31

Figure 10 AFM topographical mapping of a T1 flake. Height profile confirms flg (Z- Scale: 0-32.5

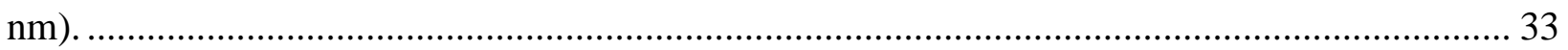

Figure 11 Schematic of an AFM Force -Distance Curve ..................................................... 35

Figure 12 Friction Force Determination [55] ................................................................ 37

Figure 13 Schematic depicting the principle of XPS ........................................................ 38

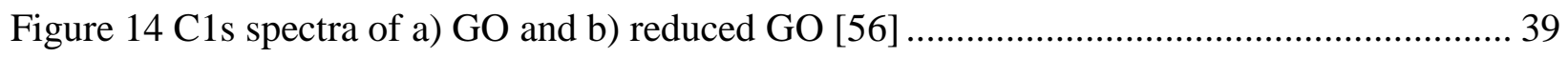

Figure 15 Force vs. distance curve obtained between the AFM tip and T1 surface .................... 40

Figure 16 Adhesion measurements for different locations on a T1 graphene flake .................. 41

Figure 17 Adhesion measurements for different locations on a T2 graphene flake ................... 42

Figure 18 Adhesion measurements for different locations on a T3 graphene surface................. 42 
Figure 19 Comparison of adhesion forces on different graphene surfaces and their underlying substrates 43

Figure 20 Comparison of the RMS roughness of the different graphene surfaces and their underlying substrates 44

Figure $21 \mathrm{C1s}$ Spectra of T1 graphene surface............................................................. 46

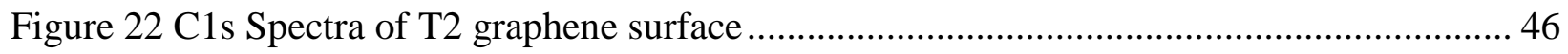

Figure 23 C1s Spectra of T3 graphene surface........................................................... 47

Figure 24 AFM plot showing pull - off points (Z-Scale: 0- 24.3nm) .................................. 49

Figure 25 Adhesion measurements for different locations on an oxidized T1 graphene flake .... 49

Figure 26 Comparison of adhesion forces between T1 and oxidized T1 graphene surfaces....... 50

Figure 27 C1s Spectra of the oxidized T1 graphene surface .............................................. 50

Figure 28 AFM topography of a T1 graphene flake divided into 8 domains (Z-Scale: 0- $193.5 \mathrm{~nm}$ ) 52

Figure 29 Adhesion measurements along a line profile from the central part of the flake to the edge in domain 5 52

Figure 30 AFM topography of a T1 graphene flake divided into 8 domains (Z Scale: 0- 49.6 nm)

Figure 31 Adhesion forces along the central part of the flake towards the edge in different domains

Figure 32 AFM topographical mapping of a T1 graphene sample (Z-Scale: 0-26.6 nm) .......... 55

Figure 33 AFM friction map of a T1 graphene sample (Z-Scale: 0-1.2V) ............................. 56

Figure 34 Friction Force vs Normal Load plot for a T1 graphene sample .............................. 56

Figure 35 Friction Force vs Normal Load plot for a T2 graphene sample .............................. 57 
Figure 36 Friction Force vs Normal Load plot for a T3 graphene sample 58

Figure 37 Comparison of coefficient of friction on different graphene surfaces and their underlying

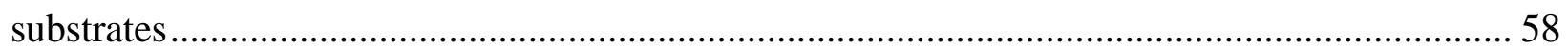

Figure 38 Friction Force vs Normal Load plot of oxidized T1 graphene sample....................... 60

Figure 39 Comparison of coefficient of friction between $\mathrm{T} 1$ and oxidized $\mathrm{T} 1$ graphene surfaces 61

Figure 40 AFM topography of a T1 graphene flake divided into 2 domains (Z-Scale: 0-53.5nm)

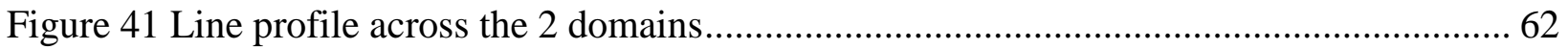

Figure 42 Friction Force vs Normal Load plot of a T1 graphene surface across 2 domains....... 63

Figure 43 Friction Force vs Normal Load plot of T3 graphene in deionized water .................... 65

Figure 44 Comparison of coefficients of friction in ambient air and deionized water ................ 65

Figure 45 Friction Force vs Normal Load plot of T3 graphene in iodine lubricated system ....... 67

Figure 46 Friction Force vs Normal Load plot of T3 graphene in BMIM PF6 lubricated system 68

Figure 47 Comparison of coefficient of friction for different ionic liquid lubrication systems ... 68

Figure 48 C1s Spectra of Iodine solution coated T3 graphene surface ................................... 69

Figure 49 Friction Force vs Normal Load plot of T3 graphene in 0.5\% (wt. /vol) NaCl system. 70

Figure 50 Friction Force vs Normal Load plot of T3 graphene in 5\% (wt. /vol) NaCl system.... 71

Figure 51 Friction Force vs Normal Load plot of T3 graphene in 7.5 \% (wt. /vol) NaCl system 71

Figure 52 Friction Force vs Normal Load plot of T3 graphene in 10 \% (wt. /vol) NaCl system. 72

Figure 53 Comparison of coefficient of friction for $\mathrm{NaCl}$ solutions of different concentrations . 72

Figure 54 AFM topography of T3 graphene coated with 5\% (wt./vol) and 10\% (wt./vol) $\mathrm{NaCl}$

solutions (Z- Scale: 0- $8.3 \mathrm{~nm}(5 \%$ (wt./vol)) and 0- $13.5 \mathrm{~nm}(10 \%$ (wt./vol))) ....................... 73 
Figure 55 Friction Force vs Normal Load plot of T3 graphene in silicone oil $(4.7 \mathrm{cP})$ lubricated system 74

Figure 56 Friction Force vs Normal Load plot of T3 graphene in silicone oil (9.2 cP) lubricated system 74

Figure 57 Comparison of coefficient of friction in water and silicone oil lubricated systems..... 75

Figure 58 C1s Spectra of Silicone oil coated T3 graphene surface ........................................ 76

Figure 59 Effect of scanning rate on friction in DI water system ...................................... 77

Figure 60 Effect of scanning rate on friction in $0.5 \%$ (wt. /vol) $\mathrm{NaCl}$ system ........................ 77

Figure 61 Effect of scanning rate on friction in $5 \%$ (wt. /vol) $\mathrm{NaCl}$ system ........................... 77

Figure 62 Effect of scanning rate on friction in $7.5 \%$ (wt. /vol) $\mathrm{NaCl}$ system ......................... 78

Figure 63 Effect of scanning rate on friction in $10 \%$ (wt. /vol) $\mathrm{NaCl}$ system ......................... 78

Figure 64 Effect of scanning rate on friction in Iodine lubricated system.............................. 78

Figure 65 Effect of scanning rate on friction in BMIM PF 6 lubricated system ......................... 79

Figure 66 Stribeck-like plot for T3 graphene in silicone oil (4.7 cP) lubricated system ............. 79

Figure 67 Stribeck-like plot for T3 graphene in silicone oil $(9.2 \mathrm{cP})$ lubricated system ............. 80

Figure 68 Schematic showing boundary, mixed, and hydrodynamic lubrication regimes [68] ... 80 


\section{LIST OF TABLES}

Table 1 Surface group distributions obtained from deconvolution of C1s XPS regions of the T1,

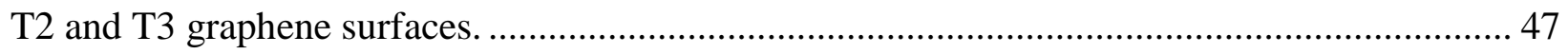

Table 2 Surface group distributions obtained from deconvolution of C1s XPS regions of the

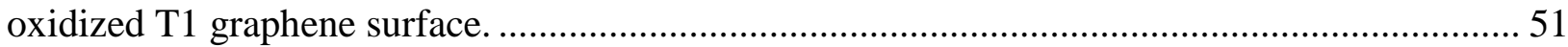

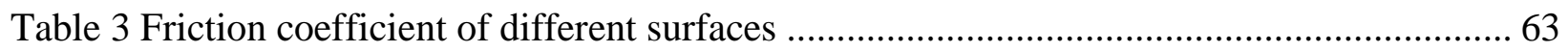




\section{NOMENCLATURE}

AFM: Atomic Force Microscopy

BMIM PF6: 1-Butyl-3-methylimidazolium hexafluorophosphate

DCE: 1, 2-dichloroethane

DI: De-ionized

DMF: Dimethylformamide

FLG: Few layer graphene

ICP-RIE: Inductively Coupled Plasma- Reactive Ion Etching

LFM: Lateral Force Microscopy

$\mathrm{NaCl}$ : Sodium chloride solution

NMP: N-Methyl-2-Pyrrolidone

RGO: Reduced graphene oxide

SiC: Silicon carbide

$\mathrm{SiO}_{2}$ : Silicon oxide

T1: Few layer graphene on $\mathrm{SiO}_{2}$ substrate (flake)

T2: Few layer reduced graphene oxide on $\mathrm{SiO}_{2}$ substrate (flake)

T3: 3 layer graphene on SiC substrate (uniform surface) 


\section{INTRODUCTION}

Graphene is a two-dimensional (2D) atomic crystal which consists of carbon atoms arranged in a hexagonal lattice [1]. It is considered to be the basic structural element of other lowdimensional carbon allotropes such as fullerenes (0D) and carbon nanotubes (1D) [2]. Its high chemical inertness, extreme strength, and easy shear capability on its densely packed and atomically smooth surface are the major favorable attributes for its impressive tribological behavior [3].

Friction, the principal cause of energy dissipation in a given mechanical system can be effectively reduced by lubrication. It is the most common means of reducing friction and lowering excessive heat generation in a mechanical system. The two-dimensional nature along with the unique friction and wear properties of graphene allow it to be used both as a solid lubricant and as an important ingredient in conformal coatings through the dispersion of graphene flakes in a solution.

The unique properties of graphene render its application in a wide range of devices and materials such as solar cells [4] [5], field effect devices, circuit boards [6], batteries [7], corrosion prevention coatings [8], ultracapacitors [9], nano-composites [10] and anti-wear materials [11] [12]. Addition of graphene platelets in oil, improved their lubrication performance [13] suggesting further applications in oil based lubricants. The integration of graphene into such devices operating under different environmental conditions makes it essential to understand the changes resulting in its tribological properties [14]. Also, the advancement of miniaturization of machines and devices 
leads to an increase in surface-to-volume ratios, due to which interfacial forces such as friction and adhesion might dominate the overall mechanical properties of the system [15].

For an insight into graphene's friction and adhesion characteristics, in the working environments of contact based microelectromechanical systems and liquid-graphene interfacial systems, we study the fundamental tribological interactions of model contacts developed between a 'single' asperity Si tip and a graphene surface in ambient air, lubricating ionic liquids and lubricating oil environments, respectively.

We report the characterization of few layer graphene (FLG) using Atomic Force Microscopy (AFM), an excellent tool for the investigation of micro/nano- tribological phenomena due to its extremely high lateral and vertical force resolutions. At its finest resolution it provides a single atom contact which can be used to determine the adhesion properties of the graphene surface. It enables to study the surface morphology and the thickness of the graphene flakes. With the help of Lateral Force Microscopy (LFM), the local frictional properties can be studied to understand the mechanical behavior under sliding contact situations both in ambient air and liquid media.

\subsection{Thesis Outline}

Following this introduction, Chapter 2 discusses the structure, properties and applications associated with graphene. Different synthesis techniques that are commonly being used to produce graphene for various applications will also be listed. A discussion about the various research activities and progress in graphene tribology since the isolation of graphene in 2004 is also included. 
Chapter 3 presents the methodology used in the research including graphene synthesis and characterization techniques.

Chapter 4 presents and discusses the adhesion results obtained between the different graphene surfaces and the 'single' asperity silicon tip in ambient air.

Chapter 5 presents and discusses the friction results obtained between the different graphene surfaces and a sliding ‘single’ asperity silicon tip in ambient air.

Chapter 6 presents and discusses the friction results obtained between the different graphene surfaces and a sliding ‘single’ asperity silicon tip in lubricating ionic liquids and silicone oil environments.

Chapter 7 concludes the thesis and discusses the future scope of work that can be carried out in this direction. 


\section{GRAPHENE SYNTHESIS AND TRIBOLOGICAL \\ CHARACTERIZATION}

\subsection{Structure, Properties and Applications}

Graphene is essentially a single atom thick, crystalline allotrope of carbon with sp2 hybridization. This can be visualized as a one atom thick chicken wire with carbon-carbon bond lengths at $0.14 \mathrm{~nm}$, interlayer bond energy of $2.5 \mathrm{eV} / \mathrm{nm}^{2}$, and interplanar spacing of $0.335 \mathrm{~nm}$ [16].

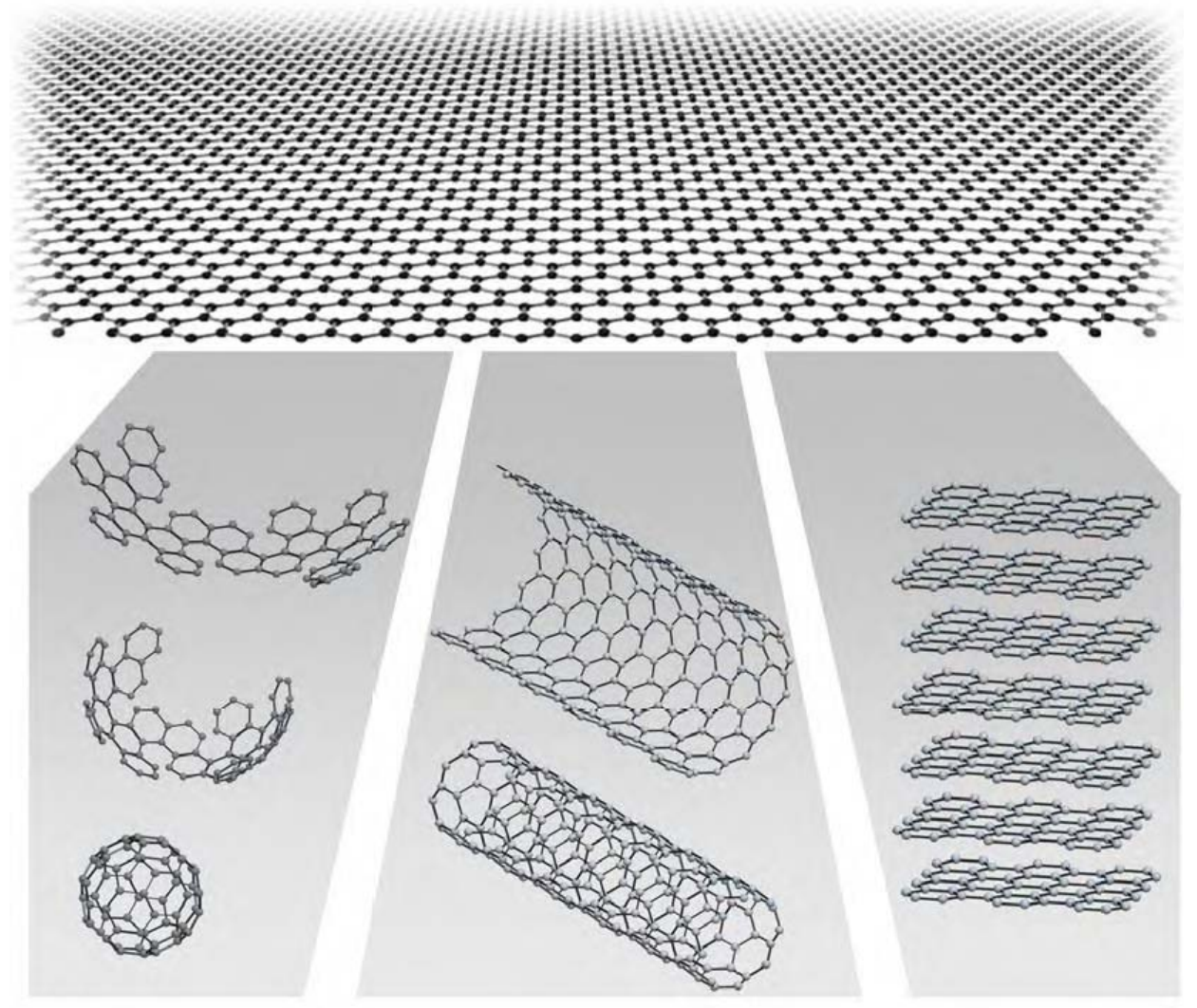

Figure 12D graphene, 0D buckyballs, 1D nanotubes and 3D graphite [2] 
Graphene’s primitive unit cell is composed of two non-equivalent atoms which span two sub lattices that are translated from each other by a lattice constant of $0.14 \mathrm{~nm}$ [17]. This is due to graphene's carbon-carbon bonds which are hybridized orbitals generated by the superposition of its 2s with 2px and 2py orbitals. These planar orbitals form the thermodynamically stable and covalent localized $\sigma$-bonds with its three nearest-neighbor atoms in the hexagonal lattice, and are responsible for most of the binding energy and for the mechanical properties of the graphene layer. The orbital left over, $2 \mathrm{pz}$, makes up the $\pi$-bond. The $\pi$-bonds hybridize together to form the $\pi$-band and $\pi$-bands. These bands are responsible for most of graphene's notable electronic properties, via the half-filled band that permits free-moving electrons [18].

The earliest references to graphene or a material like graphene were made in the 1940s, when a German scientist, Schafhaeutl, reported the intercalation (that is, insertion of a smallmolecule species, such as an acid or alkali metal, in between the carbon lamellae) and exfoliation of graphite with sulfuric and nitric acids [19].

In 1986, Boehm et al. recommended standardizing the term: "the ending - ene is used for fused polycyclic aromatic hydrocarbons, even when the root of the name is of trivial origin, for example, napthalene, anthracene, tetracene, coronene, ovalene. A single carbon layer of the graphitic structure would be the final member of infinite size of this series. The term graphene layer should be used for such a single carbon layer,” [20] [21] [22]. In 1997, IUPAC formalized these recommendations by incorporating them into their Compendium of Chemical Technology, which states: "previously, descriptions such as graphite layers, carbon layers or carbon sheets have been used for the term graphene. Because graphite designates that modification of the chemical element carbon, in which planar sheets of carbon atoms, each atom bound to three neighbors in a honeycomb like structure, are stacked in a three-dimensional regular order, it is not correct to use 
for a single layer a term which includes the term graphite, which would imply a three dimensional structure. The term graphene should be used only when the reactions, structural relations or other properties of individual layers are discussed” [19].

Graphene’s breakthrough came with the production of free-standing graphene flakes through the exfoliation of graphite crystals by Novoselov et al. [1]. Until then, the existence of a strictly 2D crystal in the free-state was thought to be not possible. K.S. Novoselov and A.K. Geim isolated free standing flakes of graphene by repeatedly peeling off highly oriented pyrolytic graphite using a scotch tape for which they were awarded a Nobel Prize in 2010 [23]. This led to a wide interest in the properties of the material. Remarkable electronic and mechanical properties, such as high charge carrier mobility $\left(>2 \times 10^{5} \mathrm{~cm}^{2} \mathrm{~V}^{-1} \mathrm{~s}^{-1}\right)$ at an electron density of $2 \times 10^{11} \mathrm{~cm}^{-2}$ [24] [25], very high thermal conductivity (over $3000 \mathrm{~W} \mathrm{~m} \mathrm{~K}^{-1}$ ) [26], and exceptional Young modulus values (> 0.5- $1 \mathrm{TPa}$ ) [27] [28] have been discovered leading to wide possibilities for potential applications.

\subsection{Synthesis Techniques}

A technique for producing high-quality graphene in large quantities is essential for further investigation of its properties and applications. Two distinct strategies have been undertaken for graphene production, i.e. the bottom-up and the top-down. The former relies on the generation of graphene from suitably designed molecular building blocks undergoing chemical reaction to form covalently linked 2D networks. The latter occurs via exfoliation of graphite into graphene [29]. The choice of graphene synthesis method is often dictated by the needs of the specific experiment or application. 
The simplest and perhaps the most popular method of graphene synthesis is the mechanical exfoliation technique. It is the method through which graphite is directly exfoliated into individual layers of carbon sheets giving graphene in both air and liquid media. It is through this micromechanical exfoliation or scotch tape method that graphene was first discovered. However, this method is limited by two factors: low output and smaller flake areas.

Graphite can also be exfoliated by utilizing ultrasound sonication in liquid environments to extract individual layers. Graphene flakes can also be produced by surfactant free exfoliation of graphite via chemical wet dispersion followed by ultrasonication in organic solvents [29].

While the above mentioned methods fall under the top-down category, there are other synthesis methods belonging to the bottom-up category which have reported large scale production of mono/few layer graphene. Among them chemical vapor deposition (CVD) on metal surfaces and epitaxial growth on $\mathrm{SiC}$ have been the most prominent.

In the CVD process, carbonaceous gaseous species react at high temperatures $\left(900 \sim 1100^{\circ} \mathrm{C}\right.$ ) in the presence of metal thin films/foils, which leads to decomposition of the carbon species and nucleation of the graphene lattice. The mechanism of graphene growth on a metal catalyst is influenced by various factors, including carbon solubility limit of the metal, crystal structure, lattice parameters, and thermodynamic parameters such as the temperature and pressure of the system [30]. Advantages of the CVD method include high quality large area graphene sheets output. Plasma-enhanced chemical vapor deposition (PECVD) has the same advantages as CVD but allows for a decrease in the deposition temperature down to $\sim 650^{\circ} \mathrm{C}$. The growth mechanism involves a balance between graphite deposition via surface diffusion and etching caused by atomic 
hydrogen. It is worth noting that the use of plasma can increase the number of defects in the graphene, which would negatively affect the tribological properties of coatings [31].

The production of graphite through ultrahigh vacuum (UHV) annealing of a SiC surface is an attractive approach, particularly for the semiconductor industry, because the products are obtained directly on $\mathrm{SiC}$ substrates and thus do not require a transfer process [32] [33]. When a $\mathrm{SiC}$ substrate is heated under UHV, silicon atoms sublimate from the substrate. The removal of $\mathrm{Si}$ leaves the surface carbon atoms to rearrange into graphene layers. The thickness of the graphene layers depends on the annealing time and temperature. The formation of flg typically requires a few minutes of annealing of the SiC surface at temperatures near $1200^{\circ} \mathrm{C}$ [34].

\subsection{Tribological Characterization:}

Liu et al. [35] investigated the nanoscale adhesive properties of graphene using both AFM and simulation tools. Silicon AFM probes were used for the adhesion force measurements on graphene synthesized by mechanical exfoliation and deposited on a $\mathrm{SiO}_{2}$ substrate in nitrogen purged environments. They reported pull-off values of $18.7 \pm 0.4 \mathrm{nN}$ for a single layer graphene surface and observed that the measurements were independent of the number of graphene layers.

Shin et al. [36] studied the frictional characteristics of exfoliated and epitaxial graphene in the micro-scale. Mechanically cleaved graphene on $\mathrm{SiO}_{2}$ substrate and epitaxial graphene on $\mathrm{SiC}$ substrate were tested using a triboindenter with a diamond probe of $1 \mu \mathrm{m}$ radius of curvature. Low friction coefficients of 0.03 for single, bilayer and tri layer graphene in ambient air were reported. The friction coefficient of pristine graphene was compared to be lower than graphene treated with 
oxygen plasma and was attributed to the surface dislocation, vacancies, or corrugation created by oxygen plasma treatment on the graphene surface.

Comparison of frictional forces between graphene and graphite by Lee et al. [37] showed that the frictional forces were independent of the normal forces and that the friction on graphene was lower than that of silicon oxide and larger than that of graphite. A negative friction coefficient, which means that friction decreases with an increase in normal load, was also observed on oxidized graphene surfaces using AFM [38].

Lee et al. [28] in the study of frictional characteristics of atomically thin sheets on silicon oxide studied mechanically exfoliated graphene deposited on $\mathrm{SiO}_{2}$ and witnessed a trend of increasing friction with decreasing thickness. However, this increasing trend of friction with thickness was absent for graphene deposited on mica, where the graphene is strongly bonded to the substrate. They explained that this effect was dependent on the adhesion force between the graphene and the substrate [39]. Loosely bound or suspended graphene sheets can pucker in the out-of-plane direction due to tip-graphene adhesion. Also, doe to the tip movement the material build up on in front of the tip which increases the contact area and also allows for further deformation of the graphene during sliding, leading to higher friction. Because thinner samples have a lower bending stiffness, the puckering effect and frictional resistance are greater for thin layers of graphene. However, if the graphene is strongly bounded to the substrate, the puckering effect will be suppressed and no thickness dependence should be observed. These results show that flg is highly suitable for the role of a lubricant with its high structural strength and low frictional characteristics. 
Ko et al. investigated the nanotribological properties of fluorinated, hydrogenated and oxidized graphenes [40]. They observed a 7-fold increase in the nanoscale friction of oxidized graphene compared to pristine mechanically exfoliated graphene deposited on a $\mathrm{SiO}_{2}$ substrate. This is attributed to the out-of plane bending deformation of graphene due to the sliding tip. They observe a change in the out of plane elastic characteristics of graphene after chemical modification and attribute it to the increase in friction for all the cases using DFT calculations.

Studies on the tribological characteristics of graphene using tight binding simulation method was reported by Bonelli et al. in 2009 [41]. According to the authors, a graphene flake with a large area experienced less friction because of the reactive atoms located at the boundary of the flake were dominant in increasing the friction. Our study attempts to experimentally observe the effects of edge-to-center distance for such a graphene flake.

The tribology of graphene has extensively been studied in vacuum and dry air environments, but little experimental work has been performed on graphene in liquids. In the micro scale, graphene sheets as lubricant additives have been used to improve the tribological properties of base oils [42] [43]. The results showed that lower concentration graphene effectively improved the tribological properties of the lubricant oils. But the interaction of graphene under liquid environments have not been studied in detail.

B. J. Robinson et al. [14] studied the nano-tribological properties of graphene in polar and non-polar liquid environments using Ultrasonic Force Microscopy and observed an increase in dodecane friction for air, water and dodecane environments respectively as shown in Figure 2. They attributed the ordering of the dodecane layer within the immediate AFM tip area that increased shear forces and friction. 


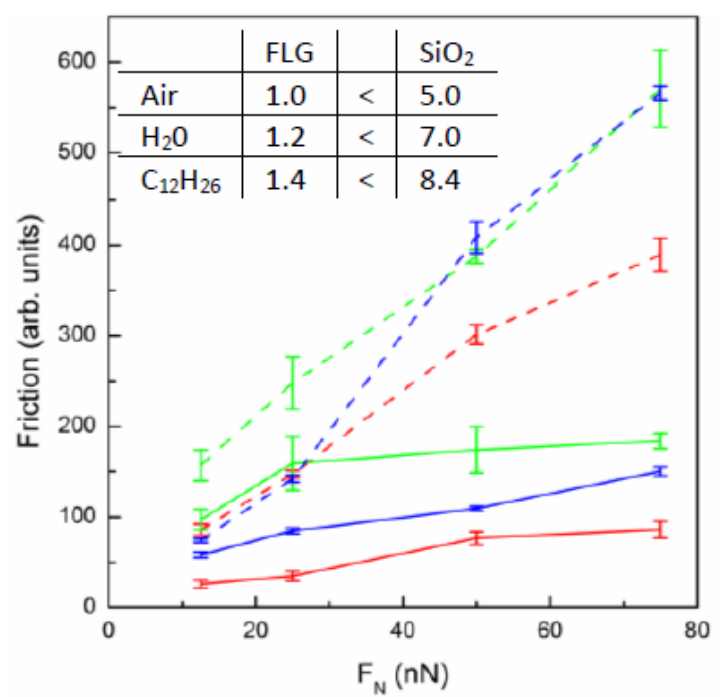

Figure 2 Relative Friction of flg (solid lines) and silicon dioxide (dashed lines) in air (red), water (blue) and dodecane (green) [14] 


\section{MATERIALS AND METHODS}

\subsection{Substrate Preparation}

To distinguish between layers of graphene, the thickness of the $\mathrm{SiO}_{2}$ substrate is important. Earlier reports suggest that at the thickness of $90 \mathrm{~nm}$ or near to $300 \mathrm{~nm}$, graphene contrast on $\mathrm{SiO}_{2}$ is maximized by about $12 \%$ at $550 \mathrm{~nm}$, where the sensitivity of human eye is optimal, resulting in a significant contrast improvement to identify graphene under optical microscopy [44] [45].

A thermal oxide layer of thickness 300nm was grown on a commercially obtained n-type Si (100) wafer. The silicon wafer was thoroughly cleaned by rinsing the wafer under deionized water. It was then immersed in ethanol and dried with nitrogen gas. The oxide layer was grown in an Oxford Plasmalab 80+ PECVD. A marked increase in contrast was observed under the optical microscope between graphene on $\mathrm{SiO}_{2}$ and graphene on $\mathrm{Si}$.

\subsubsection{Patterning of Numbers on Substrate Using Photolithography}

In order to identify the location of particular thin graphene flakes on the $\mathrm{SiO}_{2}$ substrate, a numerical grid was patterned onto the substrate. It enabled to locate the flakes during different characterization techniques. For this purpose, the process of photolithography (or "optical lithography") was used. During photolithography, parts of a thin film or the bulk of a substrate can be selectively removed and a metal contact may be deposited for example. It is a common process used in microfabrication which uses light to transfer a geometric pattern from a photomask to a light-sensitive chemical photoresist, on to a substrate. A series of chemical treatments then either engraves the exposure pattern into, or enables deposition of a new material in the desired pattern 
upon, the material underneath the photo resist. It is used because it can create extremely small patterns (down to a few tens of nanometers in size), and allows exact control over the size and shape of the fabricated objects.

A numerical grid with text $100 \mu \mathrm{m}$ in height and spaced $750 \mu \mathrm{m}$ apart was designed in-house using AutoCAD. It was then printed on a high precision transparent film photo mask at CAD/Art Services Inc., Oregon.

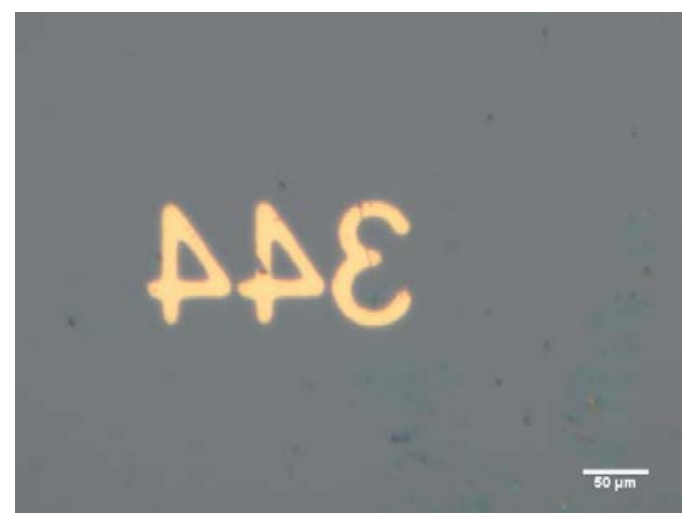

Figure 3 Optical image of prepared $\mathrm{SiO}_{2}$ substrate with gold numerical markers Scale: 50 microns $10 x$ objective

For the lift-off photolithography process, positive resist AZ 5214 was spin-coated onto the sample, illuminated and developed to expose areas for deposition. The AZ 5214 photoresist was spin coated on the substrate at $4000 \mathrm{rpm}$ for 2 minutes. The substrate was then baked on a hotplate at $115{ }^{\circ} \mathrm{C}$ for 90 seconds and let to cool down for 10 minutes. The sufficiently cooled substrate was then aligned correctly against the photomask and exposed to the UV lamp for 65 seconds under flood exposure. To develop the photoresist film, the sample was then soaked in the developer solution using AZ300 developer. Developing time was approximately 25 seconds after which it was immersed in deionized water for 10 seconds and blown dry using a nitrogen gun. 


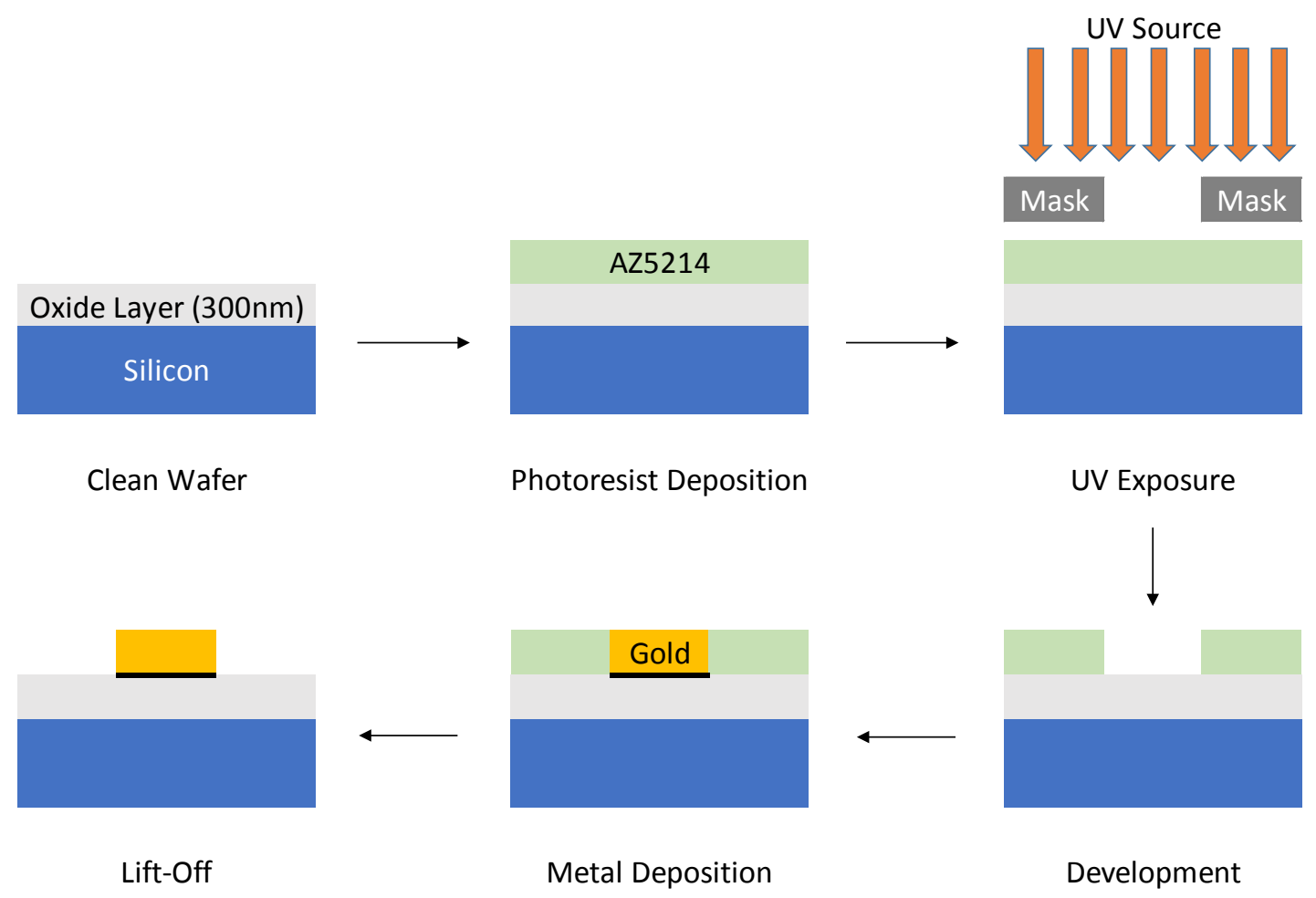

Figure 4 Schematic depicting the photolithography process

For the metal deposition, the sample was placed in an e-beam evaporator. At low pressures of approximately $1 \times 10^{-6}$ Torr, a titanium layer of thickness $10 \mathrm{~nm}$ and a gold layer of thickness $100 \mathrm{~nm}$ were sequentially deposited onto the patterns. The sample is then soaked in acetone for 1hour and ultrasonicated for a few seconds to wash away the photoresist completely and obtain a gold patterned numerical grid on the substrate as shown in Figure 3.

\subsection{Graphene Synthesis}

In our study, flg flakes to be dispersed on a silicon dioxide substrate have been produced using two techniques of the top-down approach:

(i) Liquid phase exfoliation of graphene, and 
(ii) Chemical reduction of exfoliated graphene oxide.

Another technique of the bottom-up approach was used to obtain a graphene layer of constant thickness on a silicon carbide substrate:

(iii) Using halogen based plasma etching

\section{i) Liquid Phase Exfoliation of Graphene}

Liquid-phase exfoliation has been considered as one of the most feasible approaches for industrial production of graphene due to its scalability and low cost. A large number of organic liquids including organic solvents, ionic liquids and water/surfactant solutions have been employed as exfoliation media and display a range of exfoliation efficiencies from $0.002 \mathrm{mg} / \mathrm{ml}$ to $50 \mathrm{mg} / \mathrm{ml}$ [46]. This approach typically involves sonication of graphite or graphite oxide powders in solvents.

In our study, flg flakes were produced by dispersion and exfoliation of graphite in organic solvents such as dimethyl flouridine (DMF) and 1-2- dichloro ethane (DCE). The theory behind such an exfoliation technique is that the energy which is required to break the Van der Waals forces, holding the layers of graphene together in graphite, is balanced by the solvent-graphene interaction for solvents whose surface energies match that of graphene [47].

A small amount of chemically expanded graphite flakes purchased from Asbury Carbons were heated in a conventional microwave for 20 seconds. The thermally expanded graphite in the form of ribbons was then dispersed into a solution of either 1-2-dichloro ethane (DCE) or dimethylformamide (DMF), which we call the stock solution. A glass vile containing equal parts of the stock solution and the dispersant was sonicated for 24 hours in a Branson 2510 sonicator. 
The sonication step is included to exfoliate the expanded graphite flakes into isolated, flg flakes in the liquid medium. This results in a homogeneous suspension consisting of a mixture of flg and heavier graphite particles.

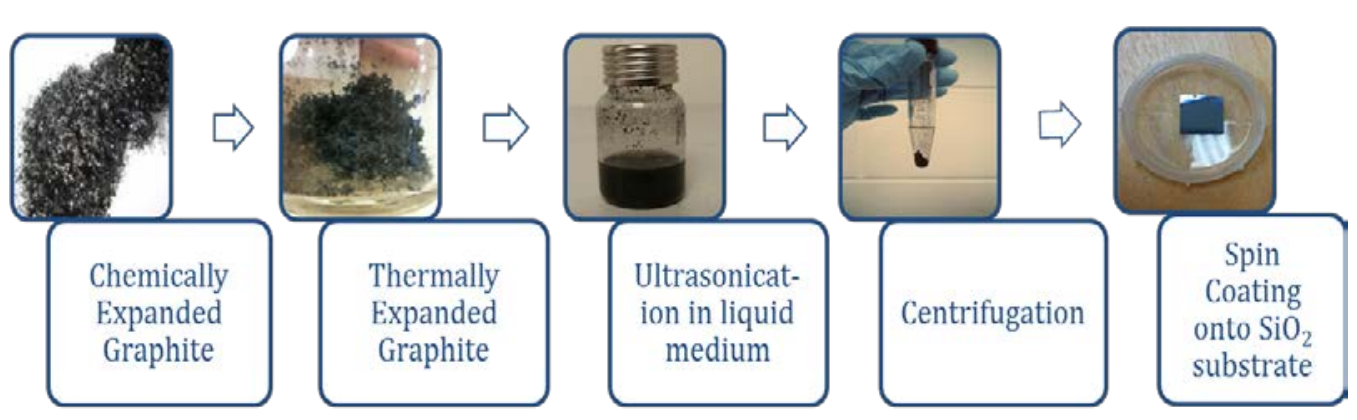

\section{Figure 5 Schematic showing the preparation of flg flakes using liquid phase exfoliation technique}

To sort the mixture, the obtained solution was centrifuged at $3500 \mathrm{rpm}$ for 30 minutes using a Fisher Scientific Centrific Model 228 centrifuge. This centrifugation step causes the heavier graphite to sediment at the bottom of the centrifuge tube and the lighter graphene flakes of few order thicknesses to rise to the top of the solution. The clear solution in the top was then pipetted into a glass vile.

The final step in the preparation of the samples is the dispersion of the graphene flakes onto the $\mathrm{SiO}_{2}$ substrate. The pipetted solution containing flg flakes was then spin coated onto the silicon dioxide wafers at 1000 rpm for 60 seconds using a Specialty Coating Systems Spin Coater Model P6700. From this point in the study, the samples produced through this technique will be denoted as "T1". 


\section{ii) Chemical Reduction of Exfoliated Graphene Oxide}

Chemically rGO in the form of an odorless black powder was purchased from Graphenea, Spain. DCE was used as a solvent but did not yield thin flakes when the previous synthesis conditions were applied. So, the rGO was dispersed at low concentrations in N-Methyl-2pyrrolidone (NMP). A glass vile containing the dispersed rGO was ultrasonicated for 30 minutes in 2 minute pulses in a Branson 2510 ultrasonicator. The homogeneous liquid solution obtained was centrifuged at $3500 \mathrm{rpm}$ for 30 minutes using a Fisher Scientific Centrific Model 228 centrifuge. The lighter rGO flakes of few order thicknesses rise to the top of the solution in the centrifuge tube. This clear solution was then pipetted into a glass vile. The solution containing reduced graphene oxide flakes was later spin coated onto the silicon dioxide wafer at $2000 \mathrm{rpm}$ for 60 seconds using a Specialty Coating Systems Spin Coater Model P6700. From this point in the study, the samples produced through this technique will be denoted as "T2".

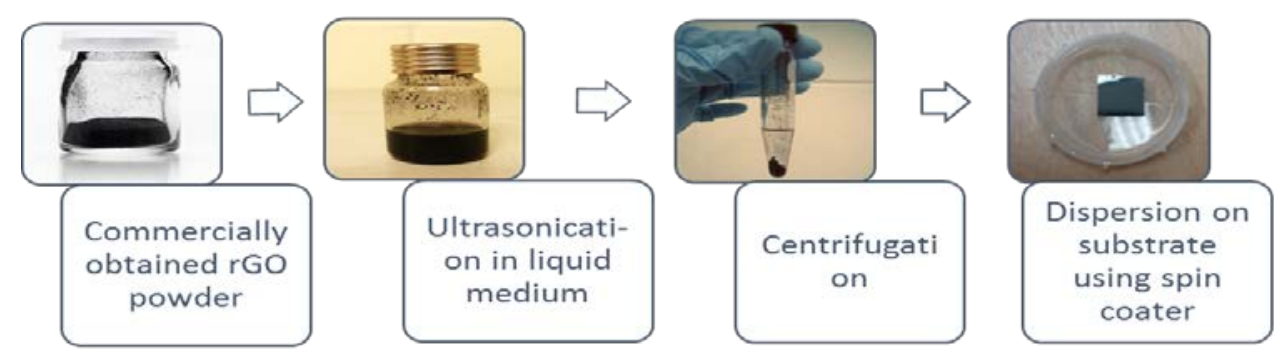

Figure 6 Schematic showing the preparation of reduced graphene oxide flakes

\section{iii) Synthesis of Graphene Using Halogen Based Plasma Etching on SiC}

For our study, 3 layer graphene on a silicon carbide substrate was provided by Dr. Charter Stinespring's lab group at Surface and Materials Studies Lab, West Virginia University. This synthesis process uses inductively coupled plasma reactive ion etching to selectively etch Si from 
the $6 \mathrm{H}-\mathrm{SiC}$ (0001) surface to produce a halogenated C-rich surface layer. Subsequently ultrahigh annealing at $970{ }^{\circ} \mathrm{C}$ was then used to nucleate and grow the graphene film.

The general synthesis process for obtaining graphene on SiC using halogen based plasma etching is as follows. 6H SiC (0001) is cleaned and plasma etched in an Inductively Coupled Plasma- Reactive Ion Etching (ICP-RIE) unit using CF4 recipes. These samples are further annealed under ultrahigh vacuum conditions at $970^{\circ} \mathrm{C}$ for 2 minutes under an $\mathrm{Ar}$ atmosphere. Following the annealing process, the samples are analyzed using XPS and AFM [48]. From this point in the study, the samples produced through this technique will be denoted as "T3".

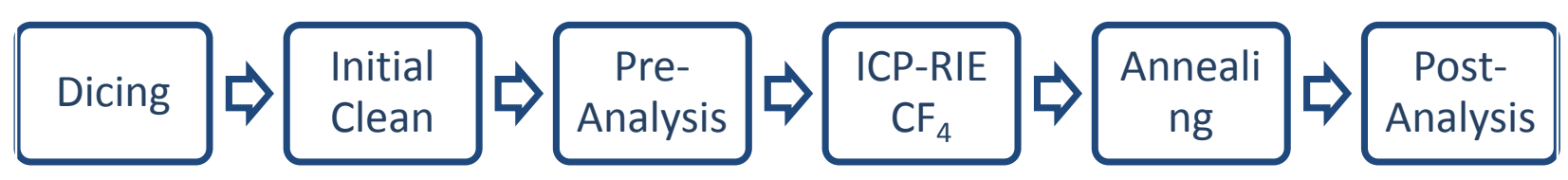

Figure 7 Schematic showing synthesis of graphene using halogen based etching on SiC [48]

\subsection{Material Characterization}

\subsubsection{Optical Microscopy}

In order to locate the graphene flakes of few nm thicknesses, optical microscopy was conducted utilizing a Laborlux 12 ME optical microscope with a 100X objective. Under the microscope, the $\mathrm{SiO}_{2}$ substrate is grey in color. The gold text is used to identify the location of the flake during different characterization techniques. 


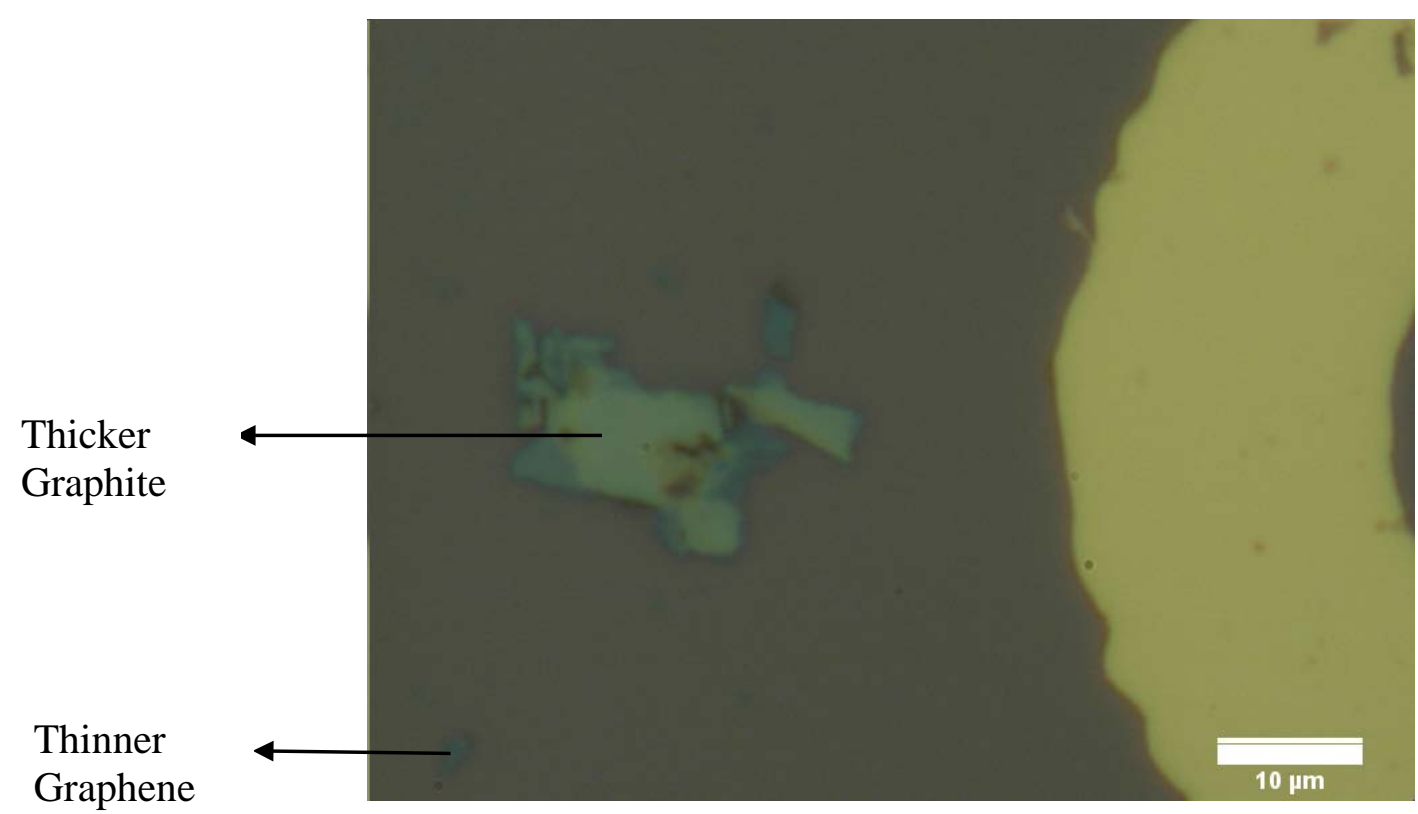

Figure 8 Optical image of a graphene flake 100X objective

The liquid phase exfoliation technique of graphene produces flakes with both thin and thick regions. Thick flakes of brighter green color have visible contrast from the wafer. Flg flakes have a darker green shade merging into the substrate and are difficult to identify. It has been reported that the contrast changes with the number of layers [45] as can be seen in Figure 8, where, thinner flg flakes are dark in comparison to the thicker bright colored flakes.

\subsubsection{Atomic Force Microscopy}

The atomic force microscope (AFM) was invented in 1986 by Binnig et al. [49]. It is a characterization tool which is essentially a combination of the principles of both the Scanning Tunneling Microscopy (STM) and the stylus profilometer that uses a sharp tip to scan the surface of the sample. The AFM gathers the surface data based on the interactions between a sharp nanometer sized probe that is exerting a force as it moves over a surface and bends in response to the force exerted between the tip and the sample's surface. 
The schematic of the AFM operation is shown in Fig.6. The AFM consists of a piezoelectric scanner that controls sub-nanometer movements in the $\mathrm{x}, \mathrm{y}$, and $\mathrm{z}$ dimensions. The sample moves under the AFM probe containing a small cantilever with a very sharp tip. This microprobe senses attractive or repulsive forces of different origin (atomic, electrostatic, and magnetic) in $\mathrm{nN}$ range causing it to deflect. A laser is focused onto the back of the cantilever coated with a reflective material. This laser beam bounces off the deflected cantilever and is directed into a split photodetector. The difference in intensity between the upper and lower windows of the photodetector is converted into a signal and sent to the computer control feedback loop.

The feedback loop attempts to maintain the probe-sample distance at a set value. Because the cantilever acts as a spring, the fixed cantilever deflection translates to a fixed probe-sample force being maintained. The amount by which the scanner has to move in the $\mathrm{z}$ axis to maintain cantilever deflection is taken to be equivalent to the sample topography. Images are then compiled line-by-line as the sample is raster scanned.

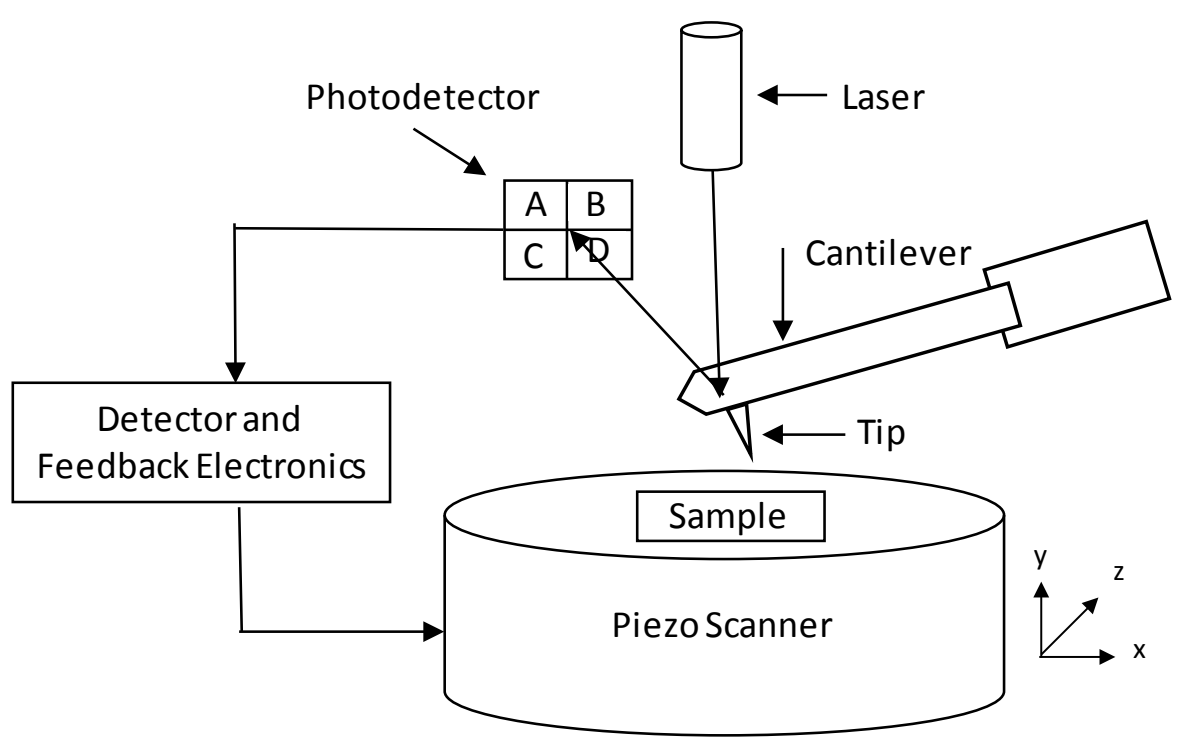

Figure 9 Schematic of an AFM operation (not to scale) 
The two most common operating modes of AFM are contact and tapping mode. In contact mode (C-M), the cantilever is moved along the surface while maintaining a constant force and the electrostatic and/or surface tension forces from the adsorbed gas layer on the surface pull the scanning tip toward the surface. Thus, contact mode imaging is heavily influenced by frictional and adhesive forces compared to non-contact or tapping mode. The tip with applied normal loads exerts high local pressures and stresses, which can damage samples or distort image data, and is therefore, used for hard samples.

In the tapping mode, the cantilever oscillates and moves over a sample and hence intermittently contacts the sample, which reduces the frictional force and contact time allowing minimal deformation of the sample, and therefore, is better suited for soft samples such as polymers and biological materials.

The AFM is not only used for surface imaging, but is also used to study different kinds of surface forces. Force spectroscopy and Lateral Force Spectroscopy are the two modes that we employ to study the adhesion and friction properties between a 'single' asperity Si tip and the graphene surface.

In this work, AFM was conducted using a Molecular Imaging PicoScan 3000 system. CONTPt-20 POINTPROBE -Silicon SPM-Sensor by Nanoworld with nominal values of $0.2 \mathrm{~N} / \mathrm{m}$ and $13 \mathrm{KHz}$ for the force constant and resonant frequency respectively were used for scanning in contact mode. The nominal tip radius was less than $25 \mathrm{~nm}$. The cantilever is coated with platinum iridium5 (PtIr5) coating on both sides. All measurements were conducted at $21 \pm 2^{\circ} \mathrm{C}$ and $45 \pm 5 \%$ relative humidity $(\mathrm{RH})$. 
C-M AFM was used to determine the thickness of the graphene flakes produced. An area of $2 \mu \mathrm{m} \times 2 \mu \mathrm{m}$ was scanned at a speed of 2.5 lines/s or $5 \mu \mathrm{m} / \mathrm{s}$. The scan angle was set to 0 and a force setpoint of $5 \mathrm{nN}$ was applied on the sample. The AFM images were then post processed using Gwyddion Version 2.40 to obtain data and present images. Figure 10 shows a typical example of a topographical image of a $\mathrm{T} 1$ graphene flake on a $\mathrm{SiO}_{2}$ substrate.
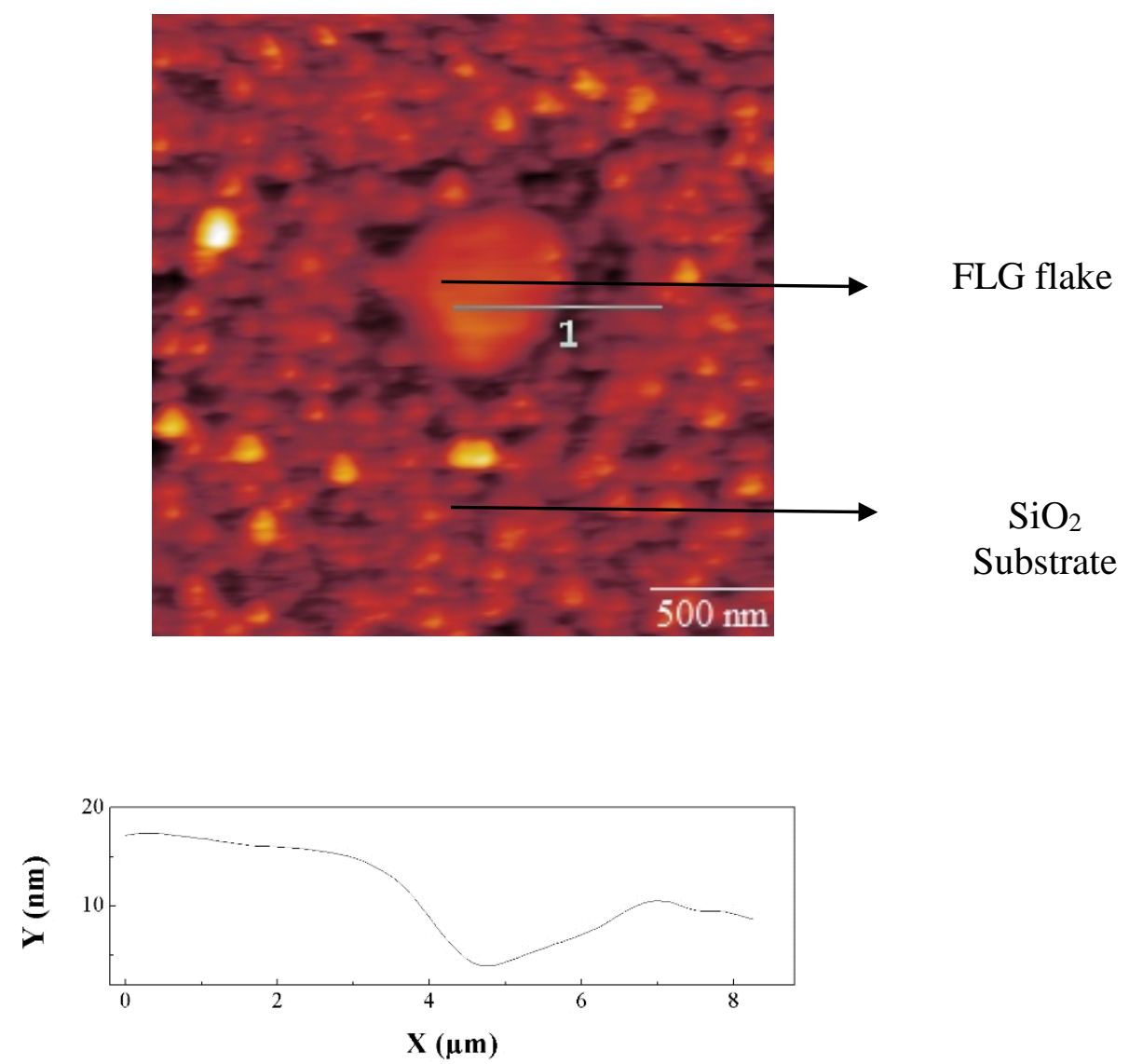

Figure 10 AFM topographical mapping of a T1 flake. Height profile confirms flg (Z- Scale: 0-32.5 nm).

The thickness of $6.7 \mathrm{~nm}$ is indicative of flg whose theoretical single layer of thickness is $0.345 \mathrm{~nm}$. According to literature, the thickness of the graphene flake measured by AFM can be exaggerated by the difference of the attractive forces between the graphene and the substrate [37]. Indeed, various groups reported different thickness values for graphene layers, with thicknesses 
ranging from 0.35 to $1 \mathrm{~nm}$, relative to the $\mathrm{SiO}_{2}$ substrate. Novoselov et al. measured platelet thicknesses of 1-1.6 nm. Gupta et al. have measured an instrumental offset induced by the AFM, of $0.33 \mathrm{~nm}$, i.e., $0.7 \mathrm{~nm}$ height for a single layer. Other authors have also reported varying step heights for flg supported on silicon oxide [50]. This variation is attributed to the change in the tip sample interaction as the tip moves over the surfaces.

\subsubsection{Methodology for Evaluating Adhesion Force using AFM}

Force spectroscopy is used to determine the adhesion force acting between the tip and sample. In this AFM mode, adhesion forces can be measured by detecting the force interaction during approach and retraction of the tip from the sample surface. During this process the height information of the z-piezo element and the vertical deflection of the cantilever detected by the four-quadrant photodiode are recorded. The result is a force-distance curve whose general features are listed as follows [51].

Initially, the probe tip is far away from the sample surface in equilibrium position. No force is detected and the cantilever remains in its undisturbed position. The forces experienced by the cantilever as it approaches from several microns above the sample surface can give information about the long range interactive forces such as electrostatic effects [A]. As the cantilever tip approaches the surface (nanometers) shorter range forces such as Van Der Waals forces and capillary forces can be measured. When these forces overcome the cantilever spring constant, the tip jumps into contact with the surface [B]. Once the tip touches the surface, the fixed end of the cantilever still moves closer to the surface producing an increase in the cantilever deflection (repulsive) from which the viscoelastic properties can be investigated [C]. 
The slope of this region gives the information regarding the elastic modulus of the cantilever. As the cantilever is withdrawn, adhesion or bonds formed during contact with the surface may cause the cantilever to adhere to the sample [D]. These adhesive forces can be measured from the force- distance curves [E]. Further retraction causes the tip to overcome the adhesive contact with the surface [F].
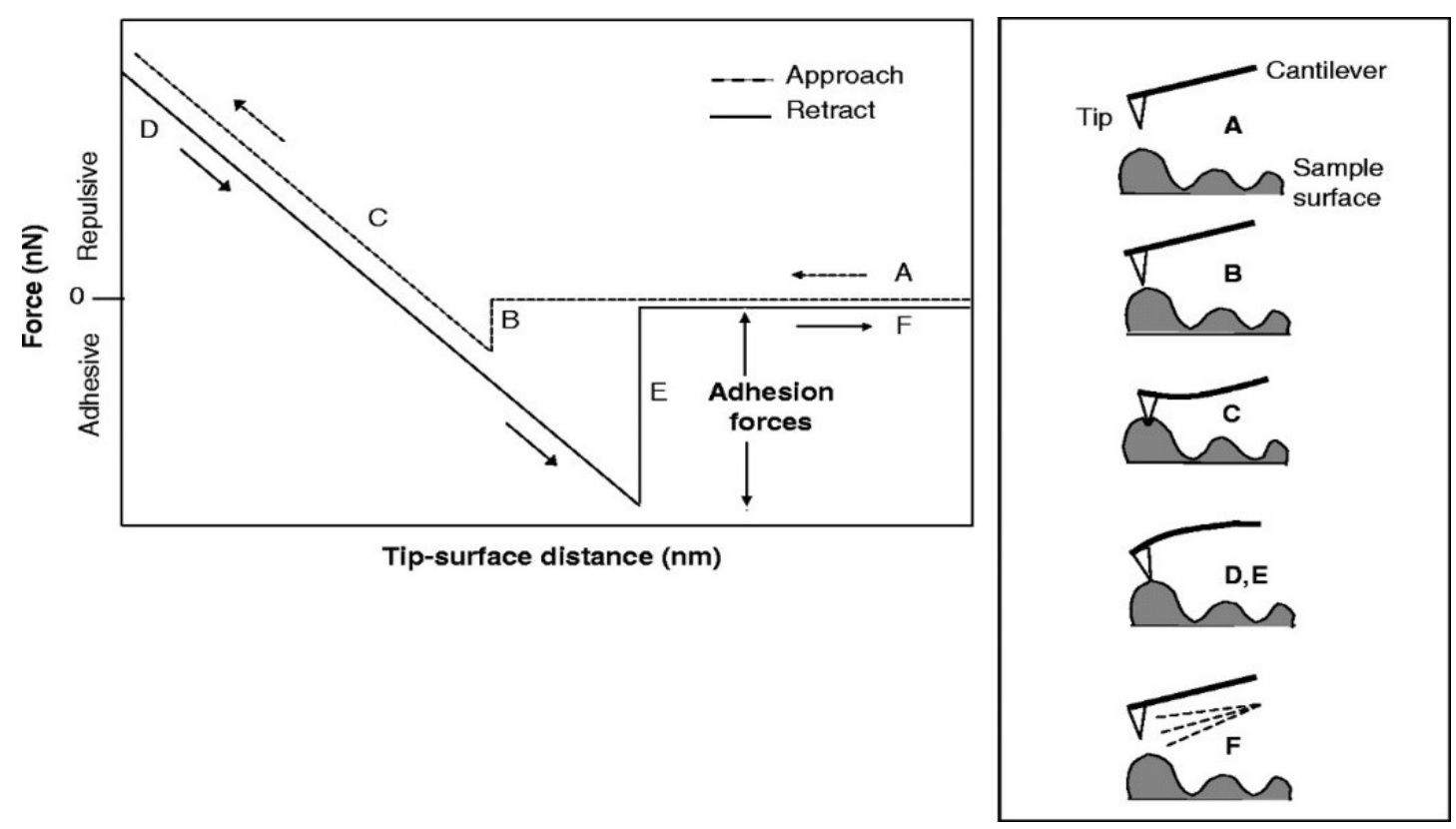

Figure 11 Schematic of an AFM Force -Distance Curve

To obtain quantitative results of adhesion forces from the measurement of the AFM cantilever deflection, the photodetector signals in volts (V) have to be converted to units of force $(\mathrm{nN})$. The force $\mathrm{F}$ between the tip and the sample is related to the cantilever's deflection through Hooke's Law

$$
F=-k x
$$

where $\mathrm{k}$ is the cantilever's spring constant and $\mathrm{x}$ is the cantilever's deflection and $\mathrm{F}$ is in the direction of the restoring force that is opposite to that of the displacement. 
The nominal values of spring constants differ from the actual values due to manufacturing variability. In order to accurately quantify the interaction between the tip and the sample, it is important to determine the spring constant of each tip used. The spring constants of individual tips were calibrated as described below.

To calibrate the spring constant $(\mathrm{k})$, force-distance curves were obtained between the tip and a clean glass sample. Three curves were averaged with sweep times set to $1 \mathrm{~s}$ each. The slope of the contact portion of the force-distance curve gives the deflection sensitivity of the cantilever. A power spectral density plot has then been obtained using Thermal $\mathrm{K}$ and the spring constants have been calculated [52].

\subsubsection{Methodology for Evaluating Friction Force using AFM}

LFM is used to determine the surface frictional characteristics of a sample by measuring the lateral bending of the cantilever. Lateral forces can arise from changes in the frictional coefficient of a region on the sample surface or from onsets of changes in height. LFM is therefore useful for measuring lack of homogeneity in surface materials and producing images with enhanced edges of topographic features.

In this work, for LFM, the scan angle of the cantilever beam was set to be perpendicular to the fast scanning direction. Friction measurements were made by applying loads in the range of 0$40 \mathrm{nN}$ to the tip travelling at speeds of $5 \mu \mathrm{m} / \mathrm{s}$ unless otherwise mentioned. The friction force was then calculated by dividing the friction signal difference between the steady-state values in the forward and reverse scans by two. The obtained value was then converted into the friction value 
by determining the torsional spring constant according to the Torsional Sader method proposed by Sader et al. [53] [54].

The coefficients of friction were obtained by plotting a linear fit for all the frictional forces obtained over the applied normal load range.
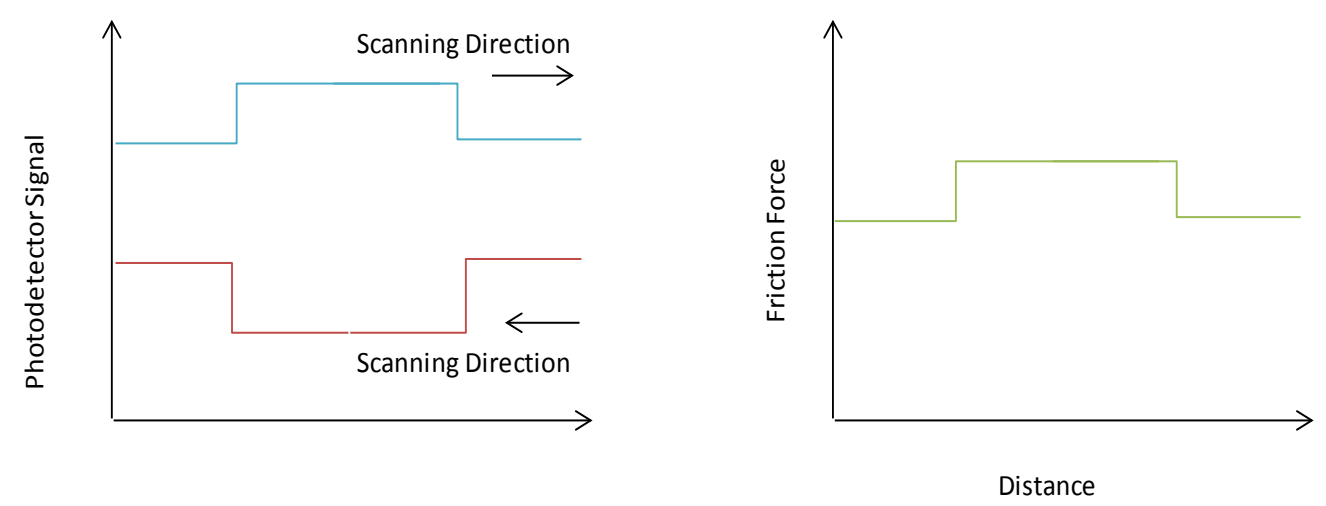

Figure 12 Friction Force Determination [55]

\subsubsection{X-Ray Photoelectron Spectroscopy}

X-Ray Photoelectron Spectroscopy (XPS) is a unique surface sensitive technique for chemical analysis. It is used to determine the elemental composition of a material. It can also determine the chemical and electronic states of the elements that exist within a material. XPS spectra are obtained by irradiating a material surface with $\mathrm{Mg} \mathrm{K} \mathrm{K}_{\alpha}(1253.6 \mathrm{eV})$ or $\mathrm{Al} \mathrm{K}_{\alpha}(1486.6$ $\mathrm{eV}$ ) X-ray photons leading to the emission of a core level electrons from the atoms in the sample. The kinetic energy, $E_{K}$, of these emitted electrons is given by

$$
\mathrm{E}_{\mathrm{K}}=\mathrm{h} v-\mathrm{E}_{\mathrm{b}}-\varphi
$$

where, hv is the energy of the $\mathrm{X}$-ray radiation, $\mathrm{E}_{\mathrm{b}}$ is the electron binding energy and $\varphi$ is the work function of the analyzer. 


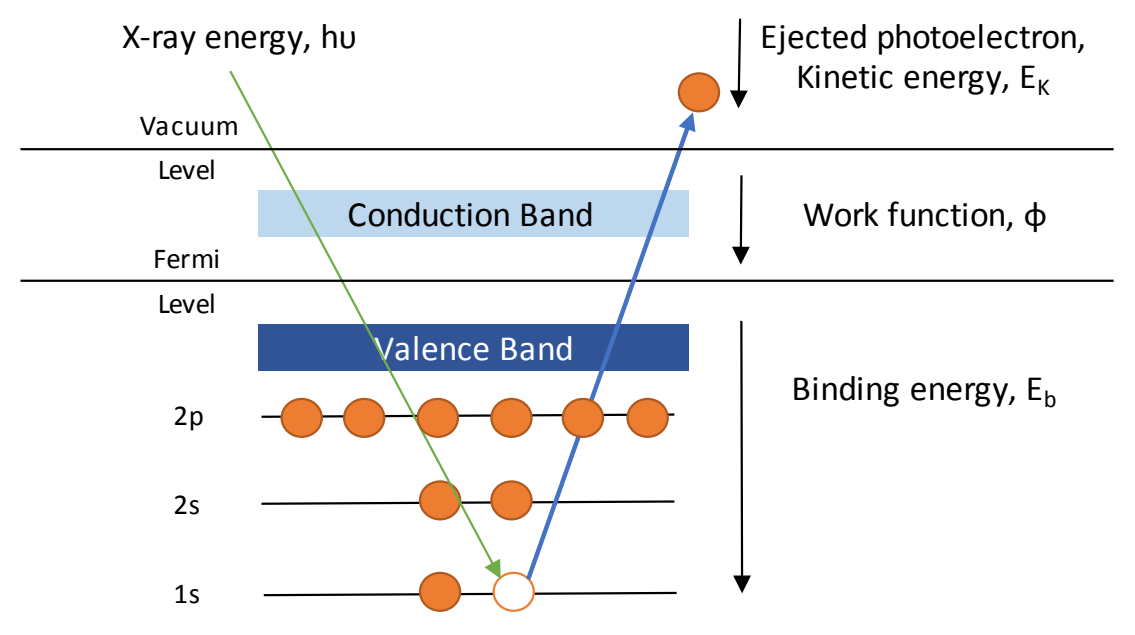

Figure 13 Schematic depicting the principle of XPS

Because each element has a unique set of binding energies, XPS can be used to identify and determine the concentration of elements in the surface region. Variations in the elemental binding energies (the chemical shifts) arise from the differences in the electron distribution associated with different compounds. These chemical shifts can be used to identify the chemical state of the materials being analyzed.Although the photons have penetrating depth in a solid on the order of 1-10 micrometers, the detected electrons only originate from a depth in the range of 0-10 $\mathrm{nm}$, due to the small inelastic mean free path of electrons in solids causing the technique to be highly surface sensitive.

The XPS analysis of the samples was conducted using a Physical Electronics PHI 5700 VersaProbe system in the surface analysis mode. The beam was $25 \mathrm{~W}$ and $15 \mathrm{KV}$ from an $\mathrm{Al} \mathrm{K}_{\alpha}$ source with a photon energy of $1486.6 \mathrm{eV}$. The emitted photoelectrons were analyzed using a hemispherical analyzer. The pass energies used for survey spectra and high resolution spectra are $117 \mathrm{eV}$ and $23.50 \mathrm{eV}$ respectively. The pressure in the main chamber during the analysis was 
always maintained below $5 \times 10^{-10}$ Torr. The acquired spectra were collected using multiple repeats and the data were averaged.

Stankovich et al. [56] studied the XPS spectra of graphene based nanosheets synthesized by the chemical reduction of exfoliated graphite oxide. We will consider this as the reference XPS spectra to verify the quality and surface elemental composition of our graphene surfaces produced.
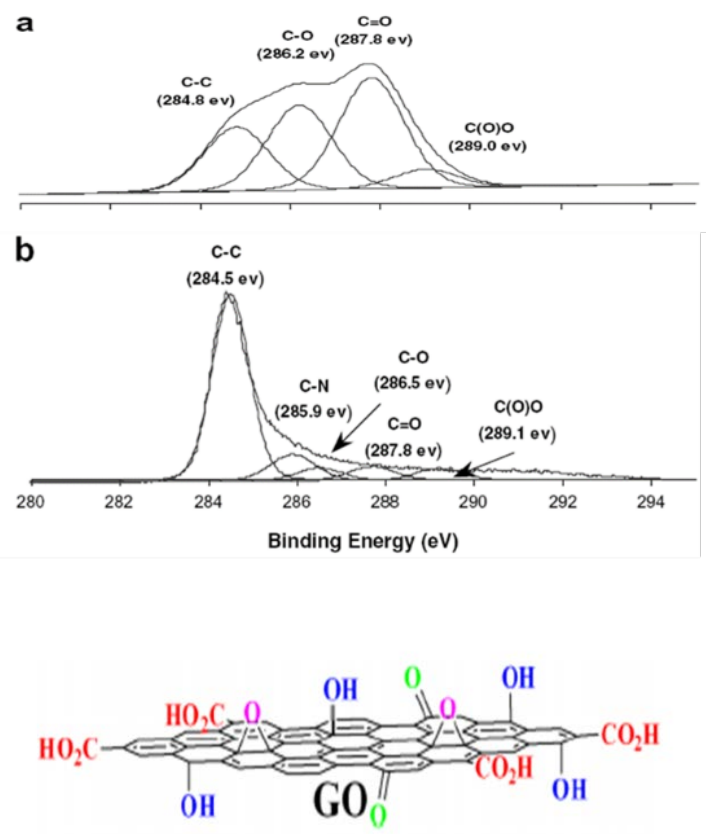

Figure 14 C1s spectra of a) GO and b) reduced GO [56]

According to literature, the C-O species include epoxide and hydroxyl groups which are distributed randomly throughout the carbon basal plane. The $\mathrm{C}=\mathrm{O}$ carbonyl groups are likely located at the edges of the graphite oxide [57]. The hydroxyl groups are thus associated with the surface defects while the carbonyl groups contribute to the edge defects due to their location. $\mathrm{COOH}$ carboxyl groups may also be present as edge defects and have a ' $\mathrm{C}$ ' binding energy of $\sim 289.1 \mathrm{eV}$. 


\section{ADHESION RESULTS}

The results of the force - distance measurements are obtained as a current (V) (the deflection of the AFM cantilever) which is converted into adhesive force using the method described in Section 4.3. A typical force vs. distance curve obtained on a T1 graphene flake are shown in Figure 15. The X-Axis represents the displacement between the tip and the surface, and the Y-Axis represents the force values measured when the tip is approaching or retracting from the surface. The red and blue curves describe the approach and retracting action of the tip respectively. The curves analyzed consist of an average of 3 sweeps at a normal load setpoint equal to $0 \mathrm{nN}$.

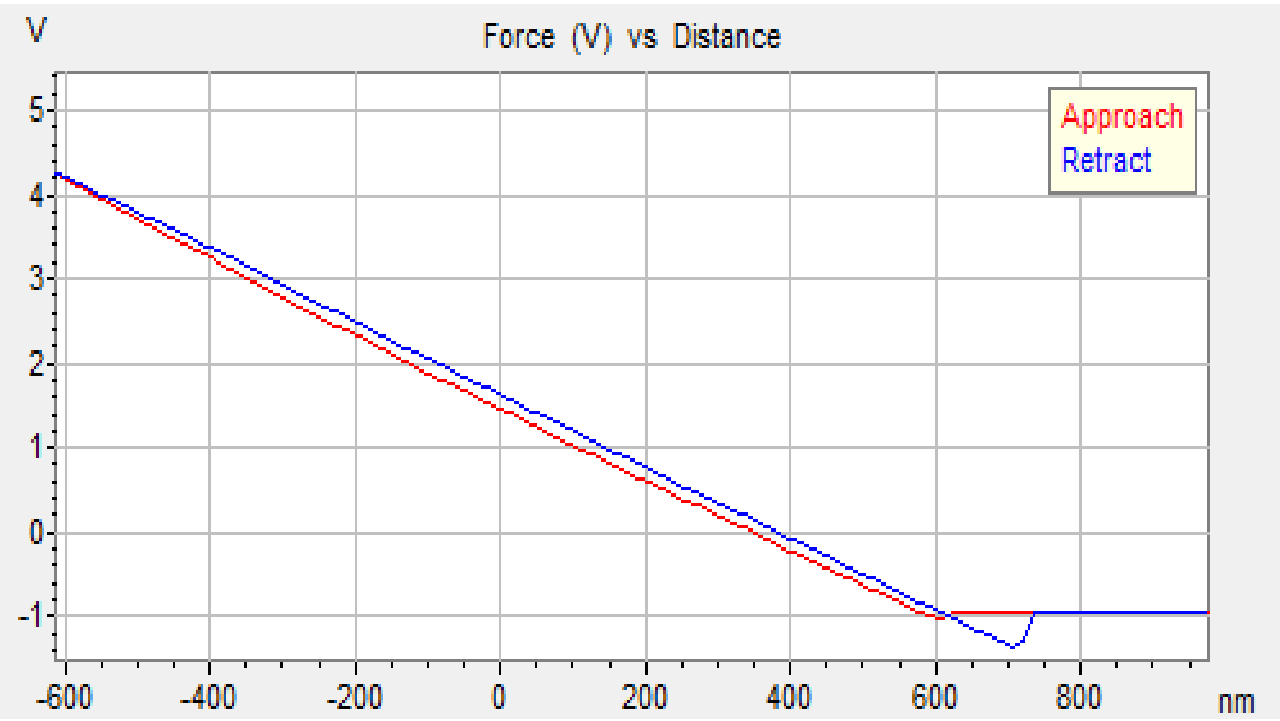

Figure 15 Force vs. distance curve obtained between the AFM tip and T1 surface 
Force spectroscopy has been performed on 9 different areas of a T1 graphene flake to obtain the adhesion values. 3 runs were performed so that the variability associated with the localized measurements can be estimated. Data from sequential approach-retract cycles at the same location, as well as for alternating between two different locations on the surface, were reproducible and yielded the same trend. The slight changes in the values of adhesion between different locations are likely due to changes in the local morphology or roughness of the substrate [35]. Figure 16 shows the adhesion values measured on a T1 graphene flake. An average adhesion force of $16.78 \pm 0.14 \mathrm{nN}$ was measured between the Si tip and a T1 graphene surface.

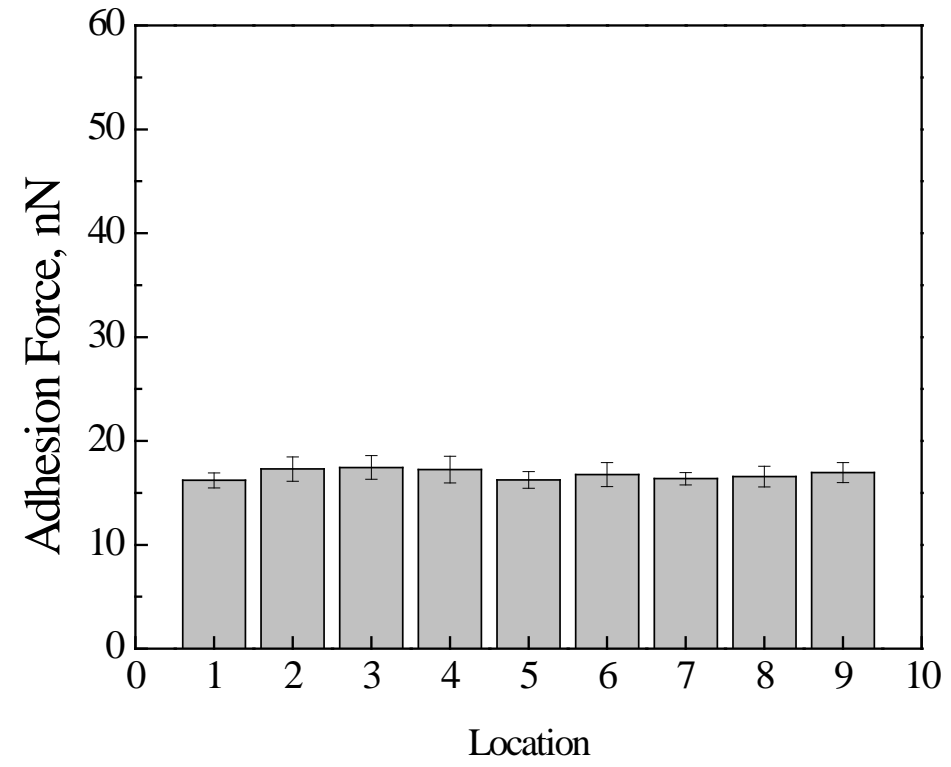

Figure 16 Adhesion measurements for different locations on a T1 graphene flake 


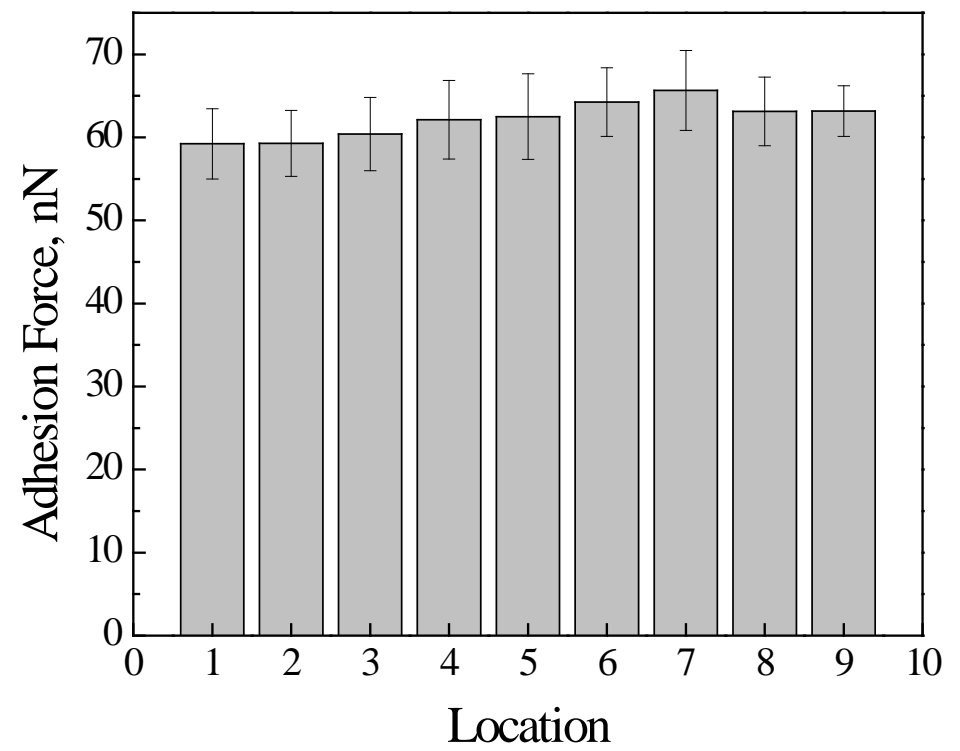

Figure 17 Adhesion measurements for different locations on a T2 graphene flake

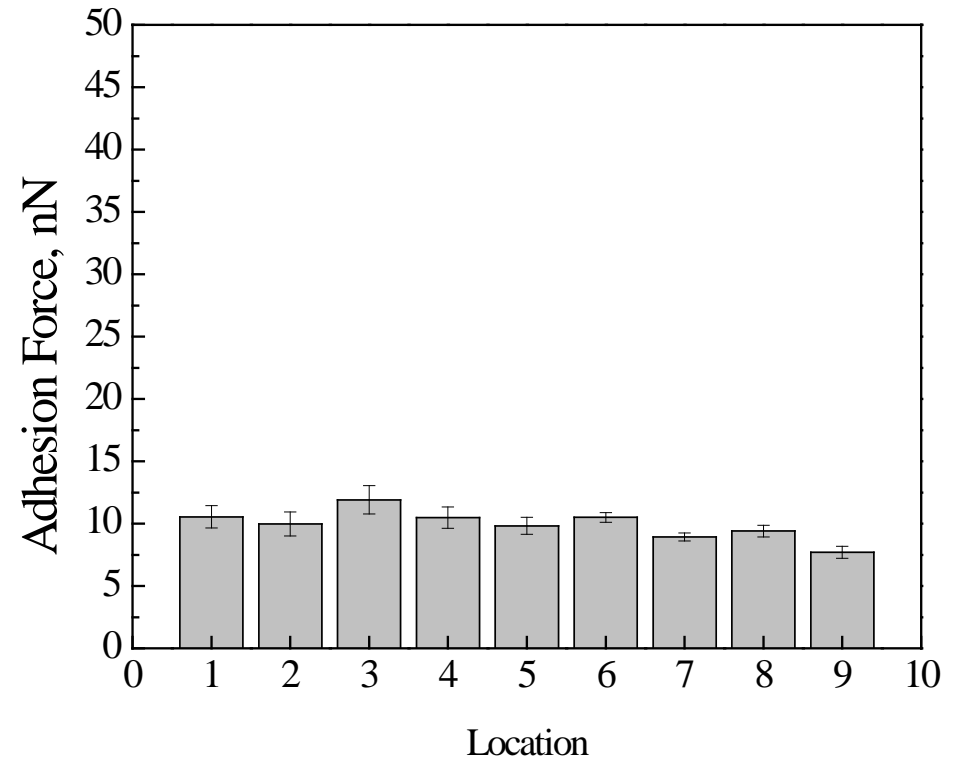

Figure 18 Adhesion measurements for different locations on a T3 graphene surface 
Force spectroscopy was then performed on a T2 graphene flake. Figure 17 shows the adhesion values measured on different locations of a T2 graphene flake. A higher average adhesion force of $62.19 \pm 0.69 \mathrm{nN}$ was measured in this case.

Force spectroscopy performed on the T3 graphene surface yielded the lowest adhesion force values of $9.19 \pm 0.37 \mathrm{nN}$ among the three graphene surfaces. Figure 18 shows the adhesion values measured on different locations of a T3 graphene surface.

Comparison of the adhesion forces on the different graphene surfaces, as well as their underlying substrates, is shown in Figure 19. The T1 and T3 graphene surfaces reduce the adhesion force of the $\mathrm{SiO}_{2}$ and $\mathrm{SiC}$ substrates respectively. The T2 graphene surface exhibits a higher adhesion force in comparison to the $\mathrm{T} 1$ graphene surface and the underlying $\mathrm{SiO}_{2}$ substrate.

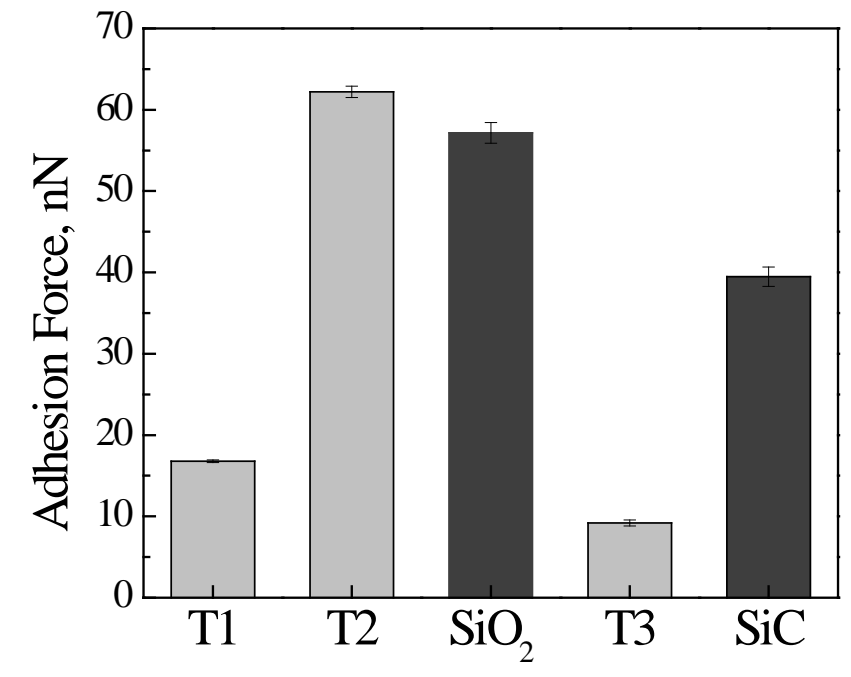

Figure 19 Comparison of adhesion forces on different graphene surfaces and their underlying substrates 
The adhesion force can be dependent upon 3 factors. 1) The interaction between the tip and the sample surface; 2) The interlayer bonding of the flg; and 3) The interfacial bonding of the graphene to the substrate.

To further understand this phenomenon, the RMS roughness of the different graphene surfaces and their underlying substrates are shown in Figure 20. From the topography scans, it was determined that the rms roughness values for the T1, T2 and T3 graphene surfaces are $0.21 \mathrm{~nm}$, $0.32 \mathrm{~nm}$ and $0.16 \mathrm{~nm}$ respectively. Their underlying substrate roughness values for bare $\mathrm{SiO}_{2}$ and $\mathrm{SiC}$ are $0.59 \mathrm{~nm}$ and $0.13 \mathrm{~nm}$ respectively.

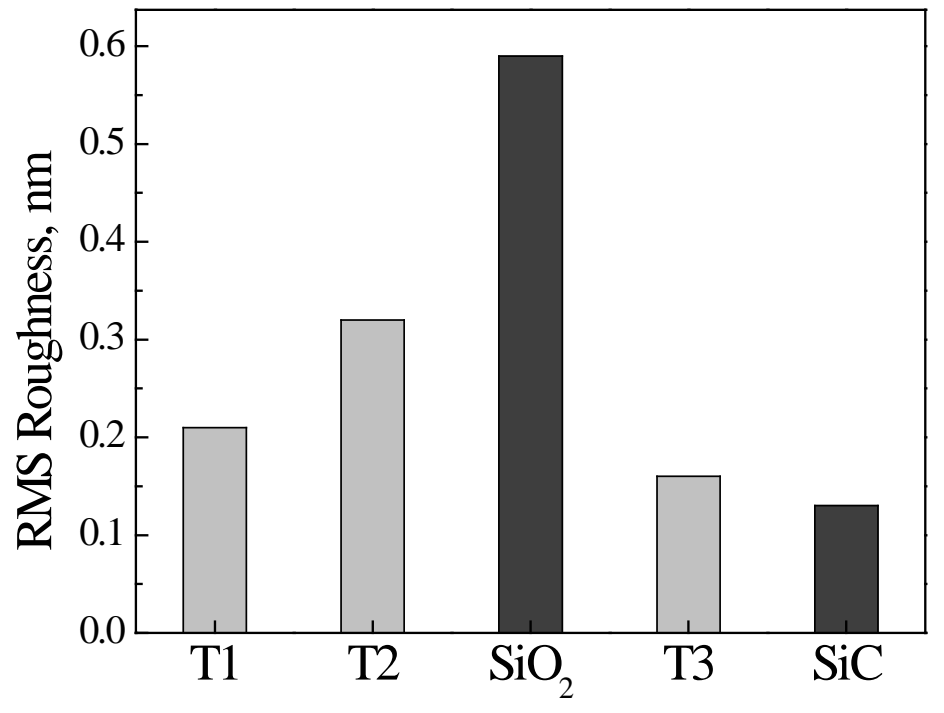

Figure 20 Comparison of the RMS roughness of the different graphene surfaces and their underlying substrates

The ability of graphene to conform extremely well to a substrate is evident from scanning probe microscopy studies of graphene on varying substrates [58]. The resulting graphene 
morphology is regulated, rather than the intrinsic random corrugations in freestanding graphene. The substrate-regulated graphene morphology results from the interplay between the interfacial adhesion and the strain energy of the graphene/substrate system [59]. The graphene-substrate interfacial bonding energy is usually weak when graphene is transferred onto the substrate (e.g. mechanical exfoliation) in comparison to being grown on it (e.g. CVD grown). The effect of the substrate roughness on the adhesion force of graphene was studied by Pourzand et al. [60] . They observed that the tip-radius scale roughness can double the adhesion force compared to a flat surface and concluded that the surface roughness significantly affects the adhesion force.

The lower adhesion values on the T3 graphene surface can thus be attributed to the low RMS roughness of its underlying substrate. It conforms well to the more uniform substrate and hence has lower adhesion values in comparison to the T1 and T2 graphene surfaces lying on the rougher $\mathrm{SiO}_{2}$ substrate.

To explain the increase in the adhesion forces between the T1 and T2 graphene surfaces, the elemental composition of the different flg surfaces have been analyzed using the XPS to better understand the adhesion forces acting between the tip and the surface in terms of the various defect concentrations. 


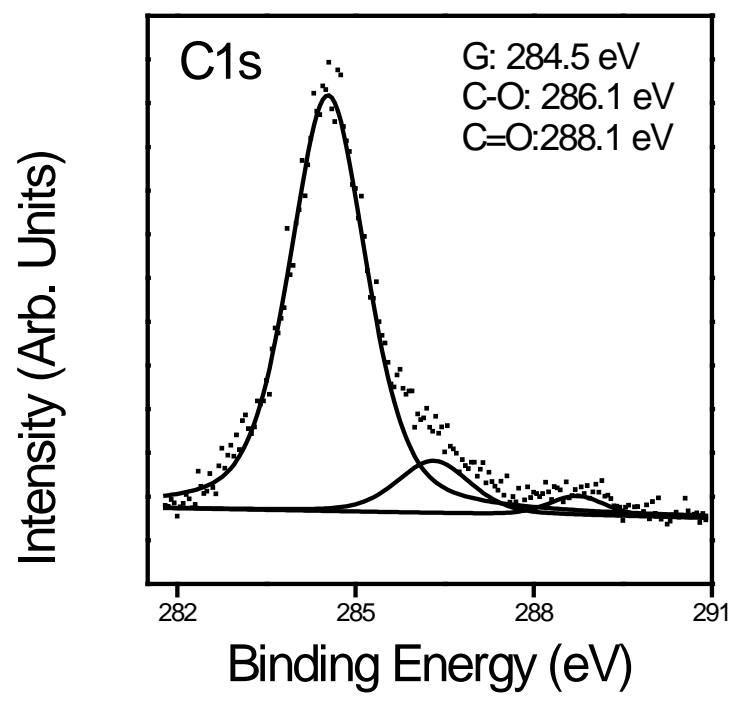

Figure 21 C1s Spectra of T1 graphene surface

The C1s core level XPS spectra for T1, T2 and T3 are shown in Figures 21, 22 and 23 respectively. Deconvolution of the C1s spectra yielded three peaks with different binding energy values representing $\mathrm{sp}^{2}$ carbon framework (C-C or graphene) at $\sim 284.5 \mathrm{eV}$ along with the presence of C-O bonds $(\sim 286.1 \mathrm{eV})$ and $\mathrm{C}=\mathrm{O}(\sim 288.1 \mathrm{eV})$.

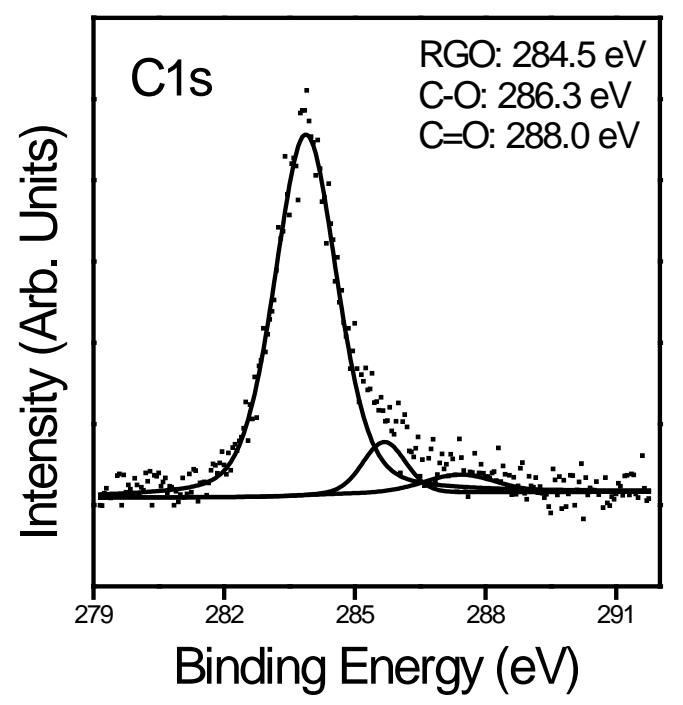

Figure 22 C1s Spectra of T2 graphene surface 


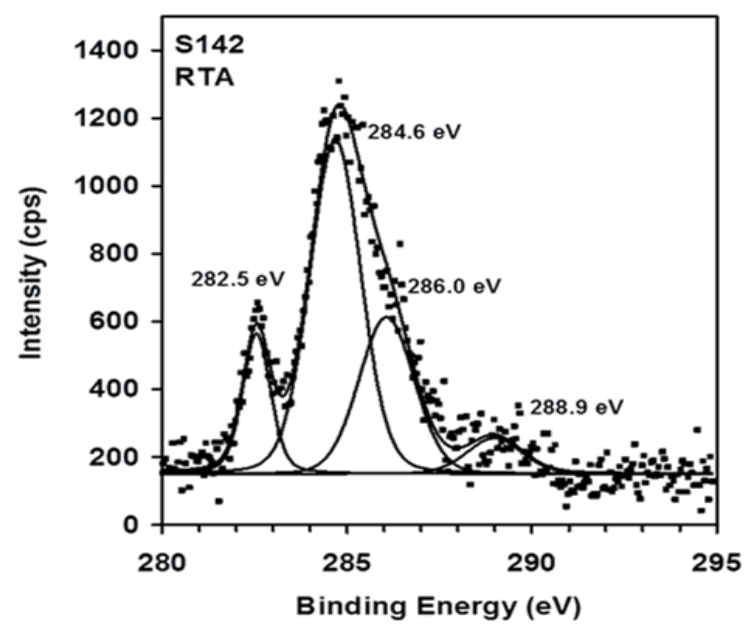

Figure 23 C1s Spectra of T3 graphene surface

\begin{tabular}{|c|c|c|c|}
\hline \multirow{2}{*}{ Assessment } & \multicolumn{3}{|c|}{ Surface Group Distributions (\%) } \\
\hline & T1 & $\mathrm{T} 2$ & T3 \\
\hline C-C (Graphene) & 77.7 & 87.6 & 61.4 \\
\hline $\mathrm{C}-\mathrm{O}$ & 19.2 & 7.8 & 14.9 \\
\hline $\mathrm{C}=\mathrm{O}$ & 3.1 & 4.6 & - \\
\hline $\mathrm{COOH}$ & - & - & 4.7 \\
\hline
\end{tabular}

Table 1 Surface group distributions obtained from deconvolution of C1s XPS regions of the $\mathrm{T} 1, \mathrm{~T} 2$ and $\mathrm{T} 3$ graphene surfaces.

The relative concentrations of surface functional groups obtained from the deconvolution of the C1s XPS regions of T1, T2 and T3 graphene surfaces are summarized in Table 1 . The concentration of the combined defects on the T1, T2 and T3 graphene surfaces are obtained at 
$22.3 \%, 12.4 \%$ and $24.2 \%$ respectively. This shows relatively good quality of the $\mathrm{T} 2$ graphene surface when compared to that of the $\mathrm{T} 1$ and $\mathrm{T} 3$ graphene surfaces.

Deng et al. [61] observed higher tip-sample pull-off forces for supported graphene relative to bare $\mathrm{SiO} 2$. The graphene was found to have a much higher density of atoms near the surface. The enhanced magnitude of the pull-off force was attributed to both the greater contact area at pull-off and the closer proximity of the surface atoms to the tip on supported graphene relative to bare $\mathrm{SiO} 2$.

In our study the increase in adhesion force of the $\mathrm{T} 2$ graphene surface in comparison to the underlying $\mathrm{SiO}_{2}$ substrate could be a result of this phenomenon. The low defect good quality T2 graphene surface has a well ordered atomic spatial arrangement when compared to the more defect prone T1 graphene surface. This could lead to an increase in contact area at the tip-sample interface thereby leading to an increase in the pull-off forces.

To further observe the role of oxygen defects on the adhesion forces of the graphene flakes, a T1 sample was oxidized at $500{ }^{\circ} \mathrm{C}$ for $10 \mathrm{~s}$. Figure 24 and Figure 25 show the AFM plot with the pull-off locations on an oxidized T1 flake and the adhesion forces measured, respectively. The magnitude of adhesion forces for the oxidized T1 flakes were found to be higher in comparison to that of the as prepared flakes as shown in Figure 26. 


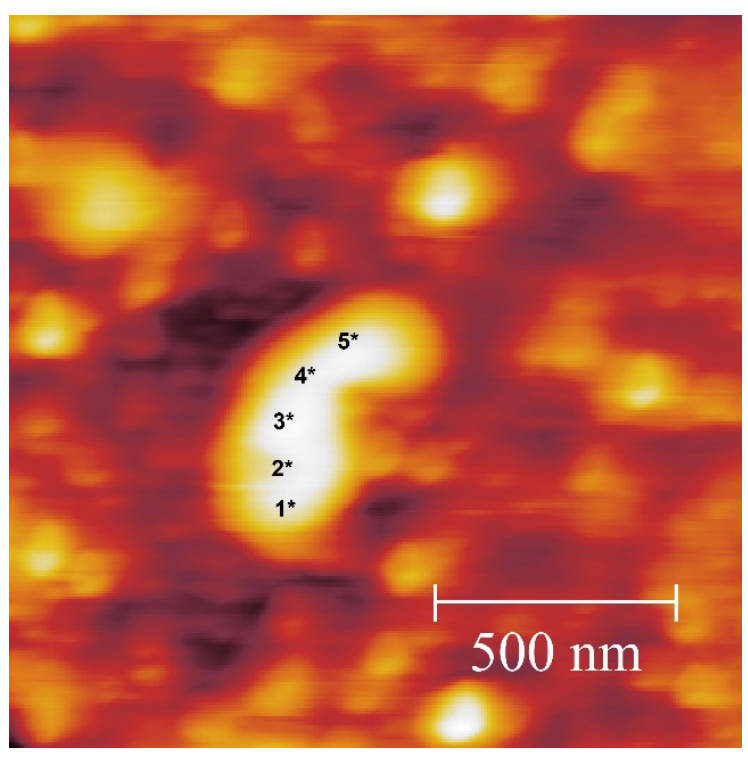

Figure 24 AFM plot showing pull - off points (Z-Scale: 0- 24.3nm)

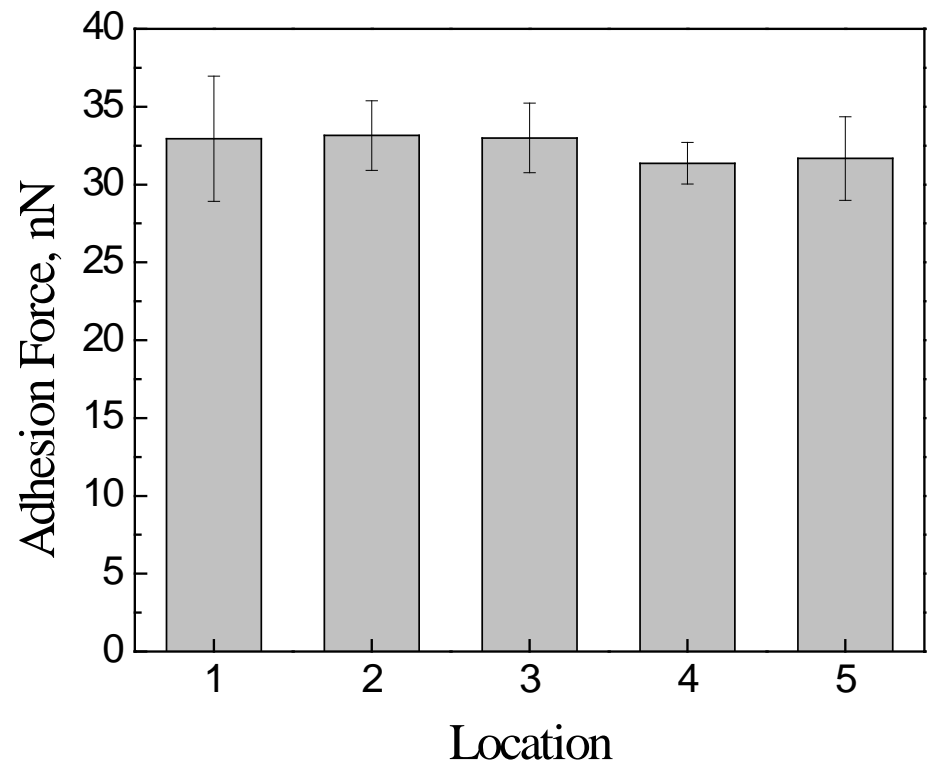

Figure 25 Adhesion measurements for different locations on an oxidized T1 graphene flake 


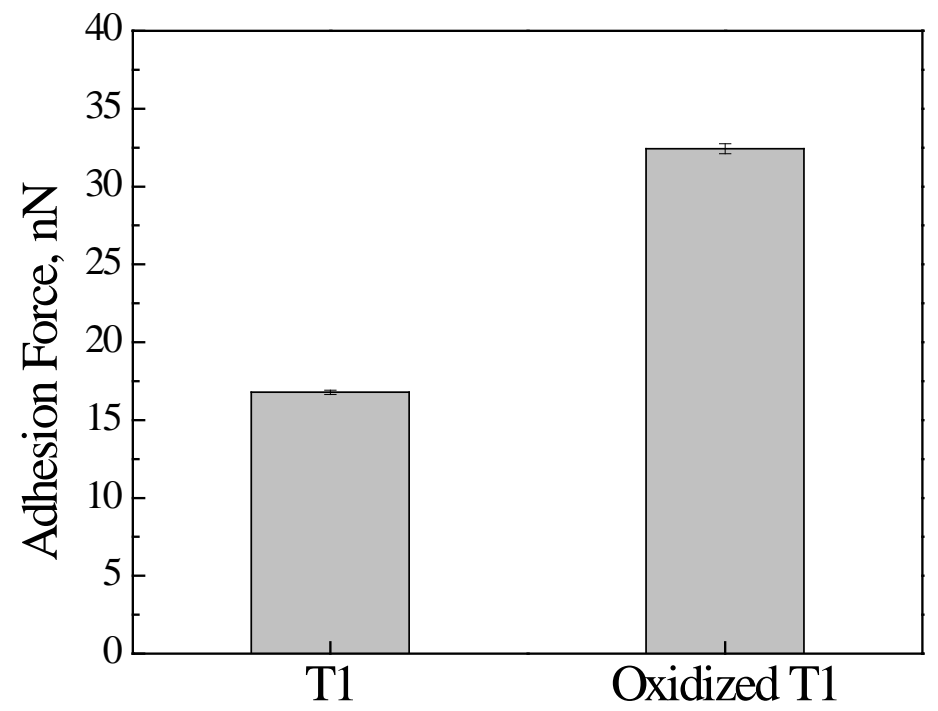

Figure 26 Comparison of adhesion forces between $\mathrm{T} 1$ and oxidized $\mathrm{T} 1$ graphene surfaces

To better understand this increase in the adhesion forces for the oxidized T1 surface, XPS spectra of the samples were analyzed. Figure 27 shows the C1s spectra of the oxidized T1 graphene surface using plasma. The surface group distributions obtained from deconvolution of C1s XPS of the oxidized T1 surface are shown in Table 2.

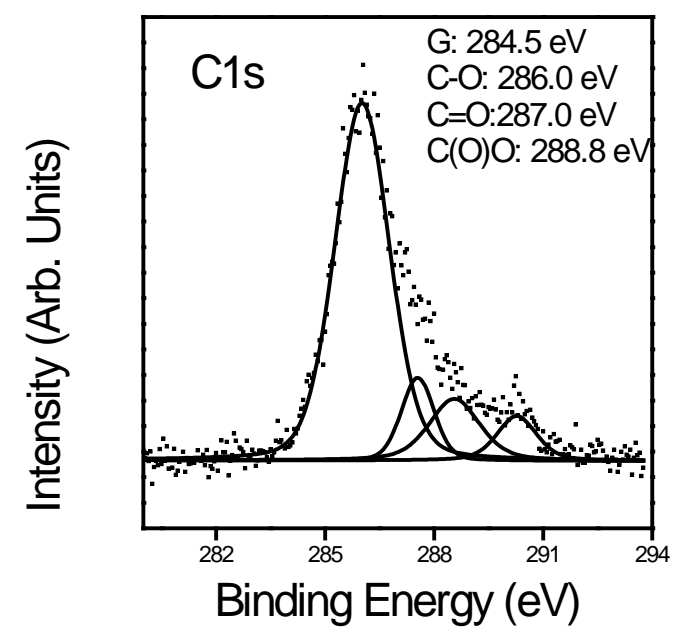

Figure 27 C1s Spectra of the oxidized T1 graphene surface 


\begin{tabular}{|c|c|}
\hline Assessment & Surface Group Distribution (\%) \\
\hline C-C (Graphene) & 70.9 \\
\hline C-O & 9.9 \\
\hline C=O & 12.3 \\
\hline $\mathrm{C}(\mathrm{O}) \mathrm{O}$ & 6.9 \\
\hline
\end{tabular}

\section{Table 2 Surface group distributions obtained from deconvolution of C1s XPS regions of the oxidized T1 graphene surface.}

We observe that the concentration of combined defects increase from $22.3 \%$ in $\mathrm{T} 1$ graphene to $29.1 \%$ in oxidized graphene. The increase in the adhesion forces for the oxidized T1 graphene surface could be attributed to such increase in the surface defect concentration. The abundant oxygenous groups such as the carboxyl and carbonyl groups at the graphene surface may give an increased hydrophilic character to it. This results in the increase of the capillary forces at the tip-sample interface. Eastman et al. reported that the capillary forces and Van Der Waals forces are found to have a strong influence at the tip surface interface [62]. This increase in the capillary forces may thus have an increasing effect on the adhesion forces.

\subsection{Edge-Centre Distance Effect on Adhesion}

To determine the effect of edge to center distance on a $\mathrm{T} 1$ graphene flake, the obtained graphene flake was divided into 8 domains as can be seen in Figure 28. The adhesion values measured from the central region of the flake towards the edge of the flake on Domain 5 is shown in Figure 29. 


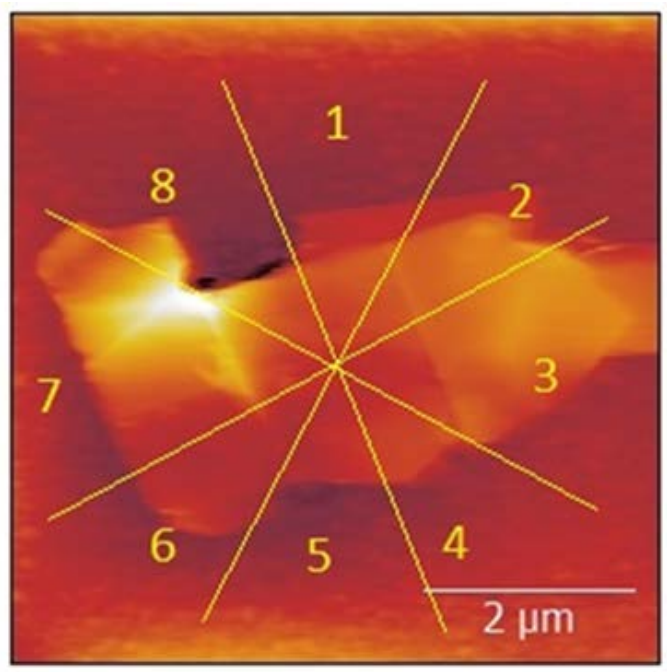

Figure 28 AFM topography of a T1 graphene flake divided into 8 domains (Z-Scale: 0$193.5 \mathrm{~nm})$
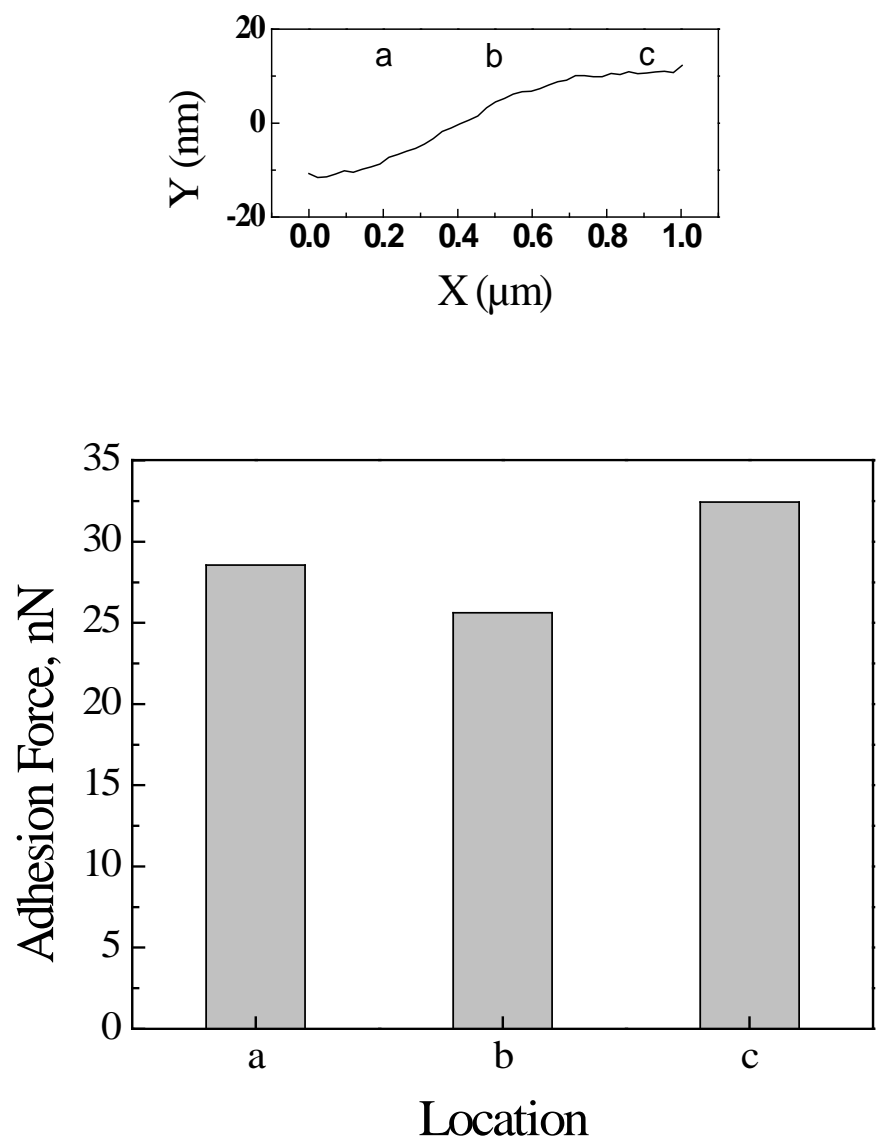

Figure 29 Adhesion measurements along a line profile from the central part of the flake to the edge in domain 5 
The three adhesion values measured on the flake show no significant trend. However, on further analysis of the variation of the adhesion force values from the central part of the flake towards an edge, a general trend of decreasing adhesion force values has been observed.

To further observe and analyze the effect, another T1 flake was divided into 8 domains as shown in Figure 30. Domains 2, 4 and 6 were chosen for their uniformity in thickness and adhesion forces, along a line profile from the central part of the flake towards the edge of the flake, were measured as shown in Figure 31.

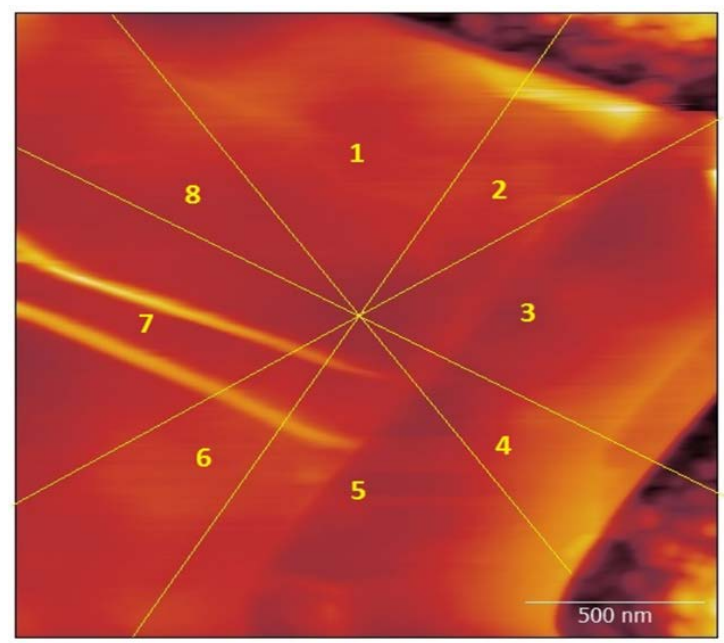

Figure 30 AFM topography of a T1 graphene flake divided into 8 domains (Z Scale: 0- 49.6 nm)

Adhesion forces along these line profiles in domains 2, 4 and 6 indicate a pattern of decreasing adhesion forces towards the edges in the case of a uniform surface area of the flake. Location 'e' in domain 4 and ' $g$ ' in domain 6 seems to be an aberration since the pull-off points being on the edge of the flake may exhibit different out of plane forces acting on the tip. 


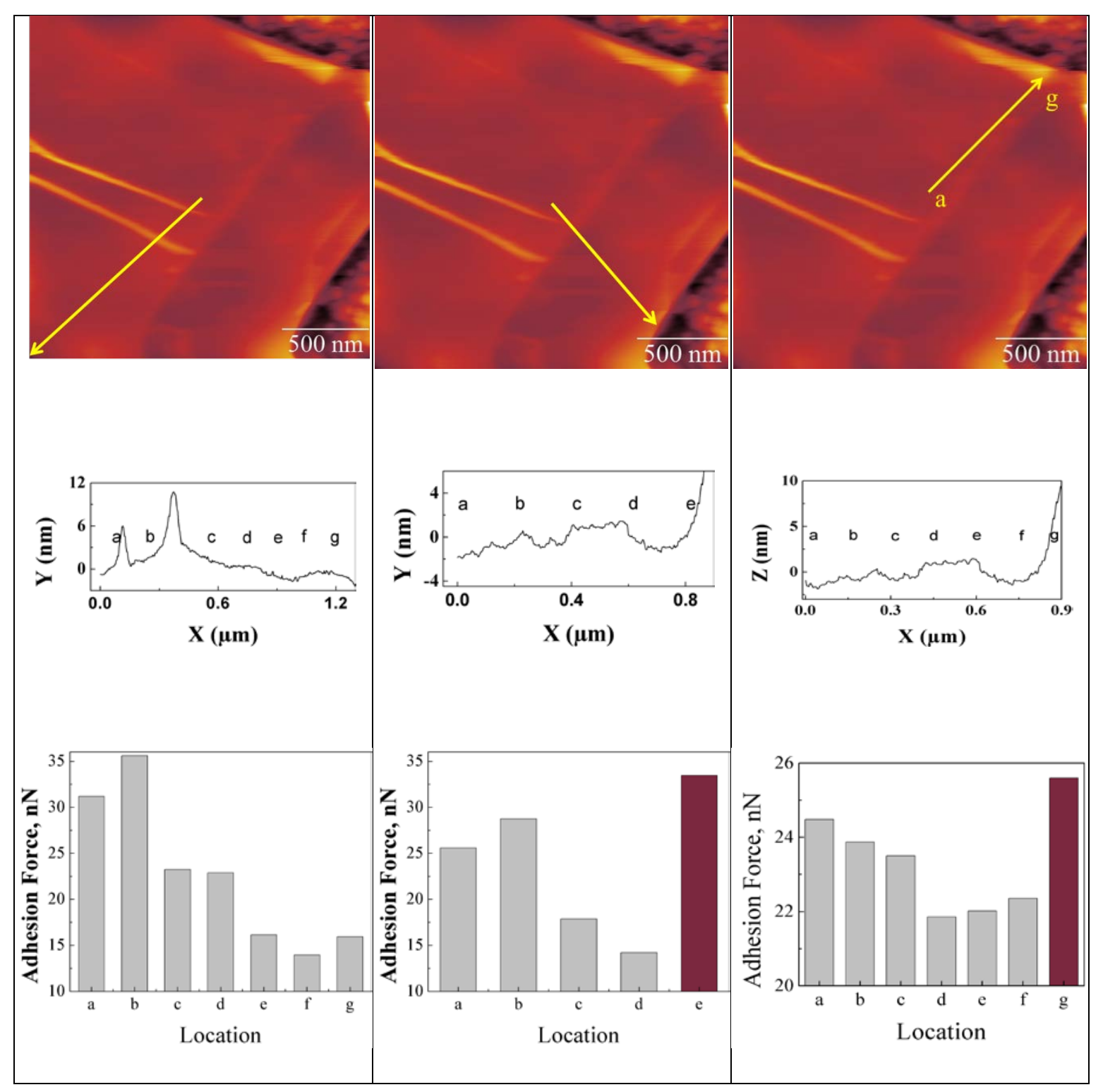

Figure 31 Adhesion forces along the central part of the flake towards the edge in different domains

Therefore we observe a pattern of decrease in the value of adhesion forces from the central part of the flake towards the edges. This can be once again attributed to the density of the atoms surrounding the tip. The central region of the flake having densely packed atoms will have more short range forces acting on the tip in comparison to the less dense atoms (and highly disordered due to edge defects) at the edges. 


\section{FRICTION RESULTS IN AMBIENT AIR}

LFM was used to determine the frictional force between the single asperity silicon tip and the graphene surfaces. An area of $5 \mu \mathrm{m}$ x $5 \mu \mathrm{m}$ was scanned at a speed of 1.497 lines/s or 7.447 $\mu \mathrm{m} / \mathrm{s}$. The scan angle was set to 0 and the range of force set points between $5 \mathrm{nN}$ to $40 \mathrm{nN}$ were applied on the sample to obtain frictional forces over a range of normal forces. Figure 32 shows the topographical image of a T1 graphene flake.

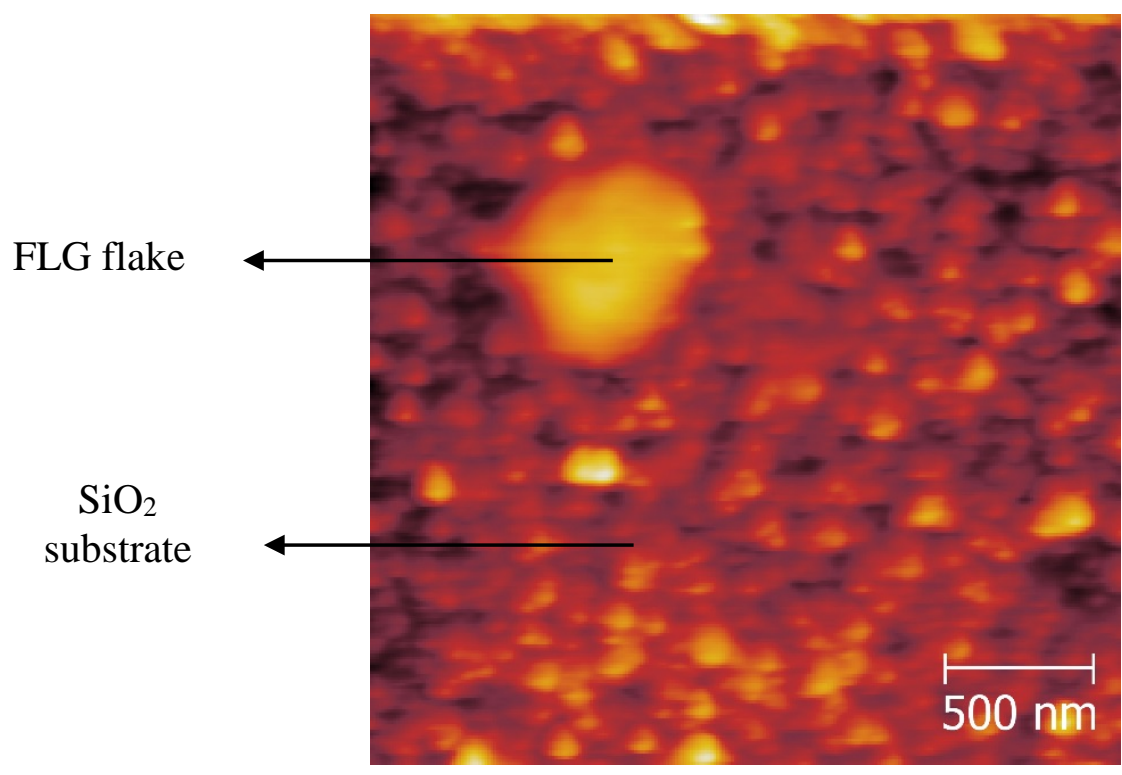

Figure 32 AFM topographical mapping of a T1 graphene sample (Z-Scale: 0-26.6 nm)

Figure 33 shows a typical friction map measured simultaneously with the topography. The frictional forces are obtained from these friction maps in terms of electric signals $\mathrm{V}$. These are then converted into units of force in $\mathrm{nN}$ according to the method outlined in Section 4.3. The slope of a linear fit through the frictional forces over the range of loading conditions gives the coefficient of friction value. 


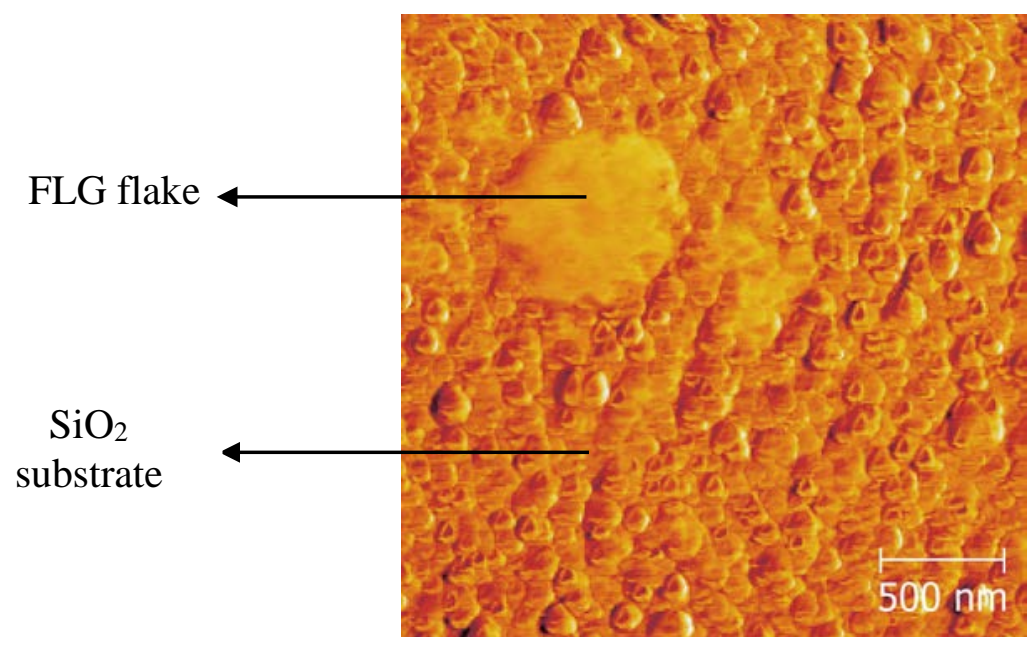

Figure 33 AFM friction map of a T1 graphene sample (Z-Scale: 0-1.2V)

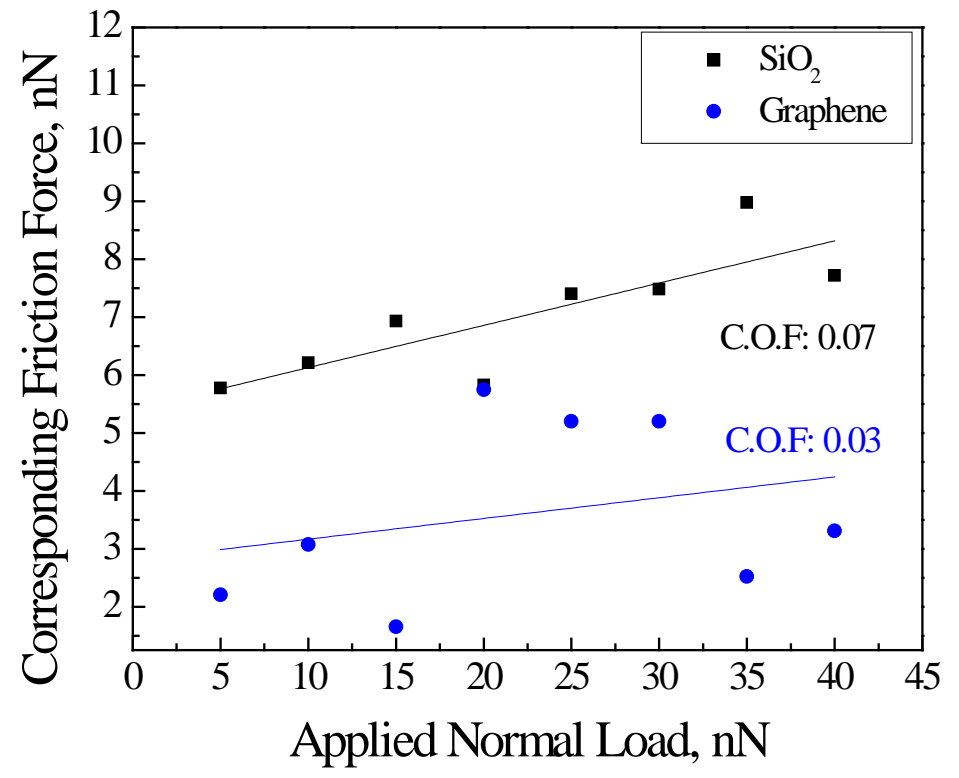

Figure 34 Friction Force vs Normal Load plot for a T1 graphene sample 
The coefficients of friction for the $\mathrm{T} 1$ graphene flake and the bare $\mathrm{SiO}_{2}$ substrate were found to be $0.03 \pm 0.005$ and $0.07 \pm 0.01$ respectively. The difference in friction values obtained for the same applied loads on different surfaces can be seen in Figure 34. Similar friction maps were obtained for $\mathrm{T} 2$ and $\mathrm{T} 3$ graphene surfaces as well as a bare $\mathrm{SiC}$ substrate and their coefficients of friction values were found to be $0.06 \pm 0.01,0.04 \pm 0.003$ and $0.058 \pm 0.004$, respectively. The Figures 35 and 36 show the friction plots obtained for T2 and T3 graphene surfaces. It can be seen that the friction increases with the increase in applied normal loads for all measured samples.

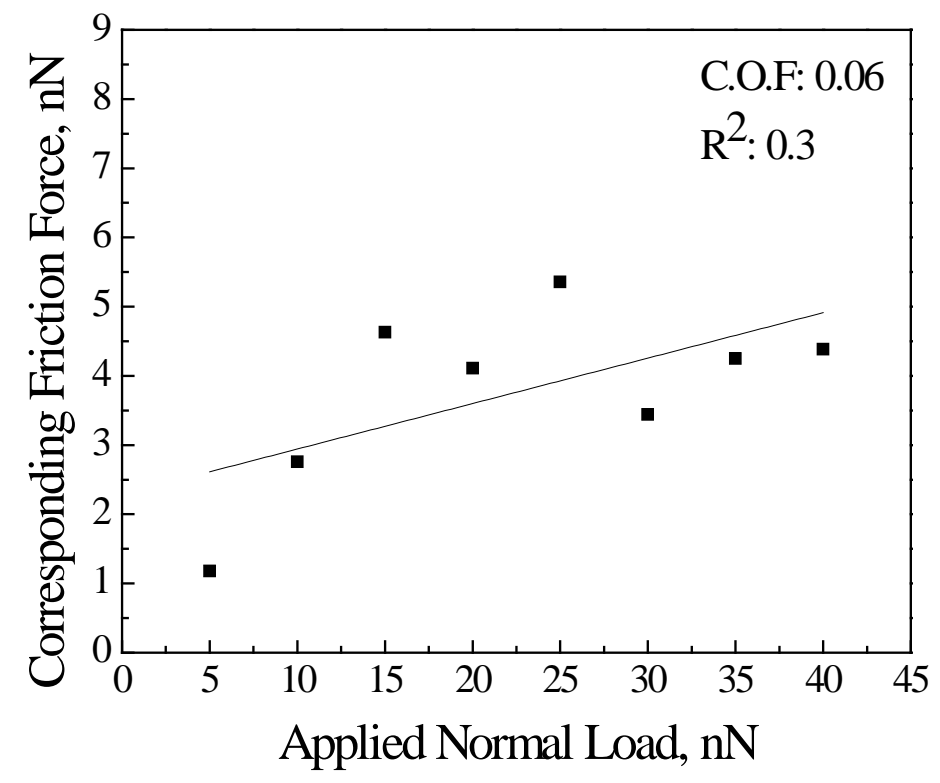

Figure 35 Friction Force vs Normal Load plot for a T2 graphene sample 


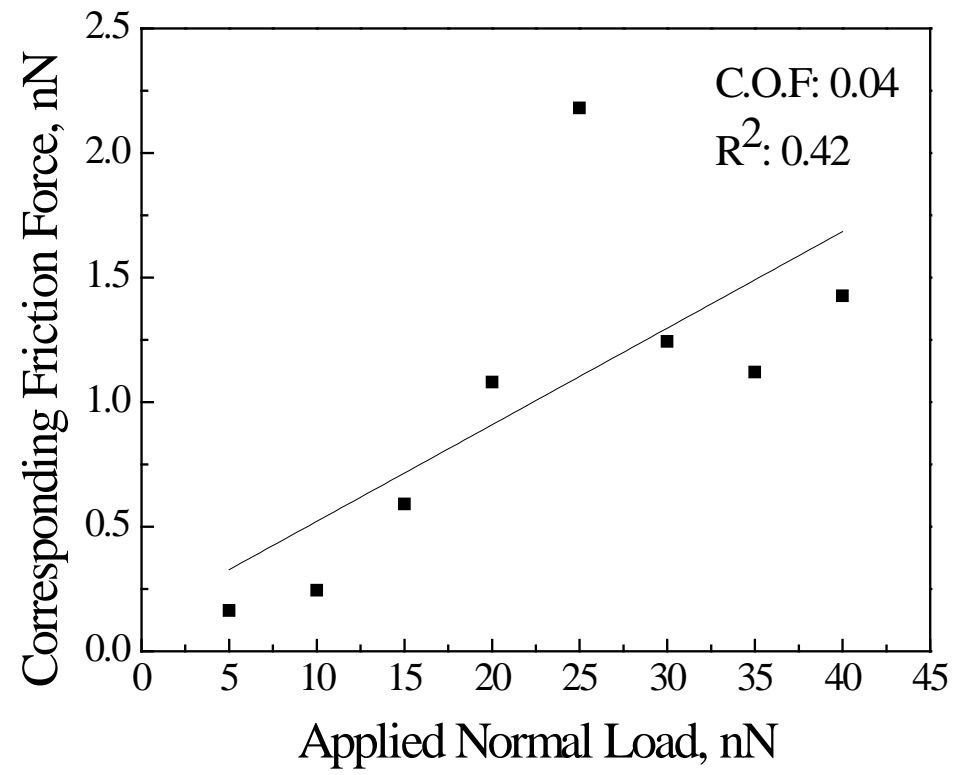

Figure 36 Friction Force vs Normal Load plot for a T3 graphene sample

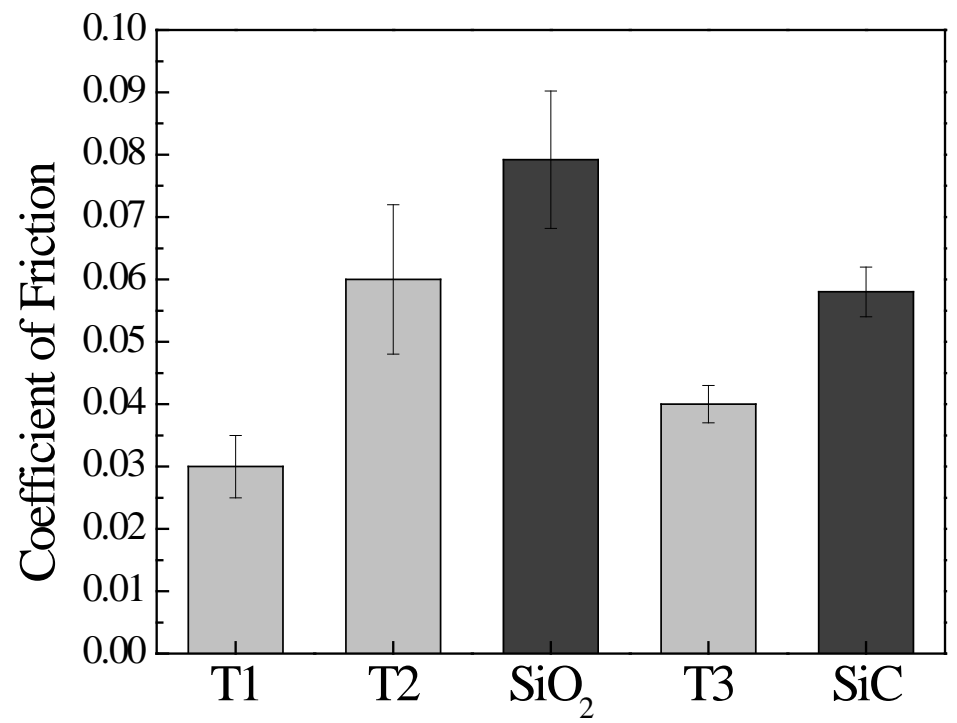

Figure 37 Comparison of coefficient of friction on different graphene surfaces and their underlying substrates 
Figure 37 shows the comparison of the coefficient of friction values frictional for the different flg surfaces and their underlying substrates.

In all the measurements, the coefficient of friction values obtained for the flg surfaces were found to be lower than that of their underlying substrates making them ideal candidates for surface coatings in NEMS/MEMS devices to reduce the friction between Si surfaces in contact. The bare $\mathrm{SiO}_{2}$ substrate presents the highest friction and coefficient of friction values among all the surfaces. The T3 graphene surface has the lowest values of frictional forces when compared to the other flg surfaces.

The higher friction values between the tip and the T1, T2 graphene surfaces in comparison to that of the T3 graphene surface can be attributed to the underlying substrate roughness. Dong [63] conducted molecular dynamics simulations to study friction on graphene and its thickness dependence. It was found that substrate roughness is the most dominant mechanism and can significantly increase friction. A graphene sheet can conform to a rough substrate due to adhesion and normal load. The conformation will induce roughness in graphene and cause friction enhancement. The puckering effect comes into play when substrate is atomically smooth such as graphite and mica. Its effect on friction is moderate compared with substrate roughness. The higher roughness value of the $\mathrm{SiO}_{2}$ substrate than that of the $\mathrm{SiC}$ substrate as shown in Figure 20 contributes to the enhanced friction values between the tip and the T1, T2 graphene surfaces in comparison to that of the T3 graphene surface.

The defect concentration of the carbonyl groups contributing to the edge defects is higher in the case of the T2 graphene surface than that of the T1 graphene surface as shown in Table 1. This increased concentration of the edge effects contribute to the enhancement of the frictional 
forces when the tip slides on the graphene surface. Thus we see an increase in the frictional values between the tip and the $\mathrm{T} 2$ graphene surface when compared to that of the T1 graphene surface.

To further study the effect of oxygen based defects on the friction values. Lateral force microscopy was performed on an oxidized $\mathrm{T} 1$ graphene surface.

The coefficient of friction of the oxidized T1 graphene sample was determined to be 0.23 \pm 0.028 . Figure 38 shows the friction plot obtained for an oxidized T1 graphene surface. The friction values obtained are higher in magnitude for the oxidized $\mathrm{T} 1$ graphene surface in comparison to that of the as produced T1 graphene surface. Figure 39 shows the increase in the coefficient of friction values between the oxidized T1 graphene surface and the as prepared T1 graphene surface.

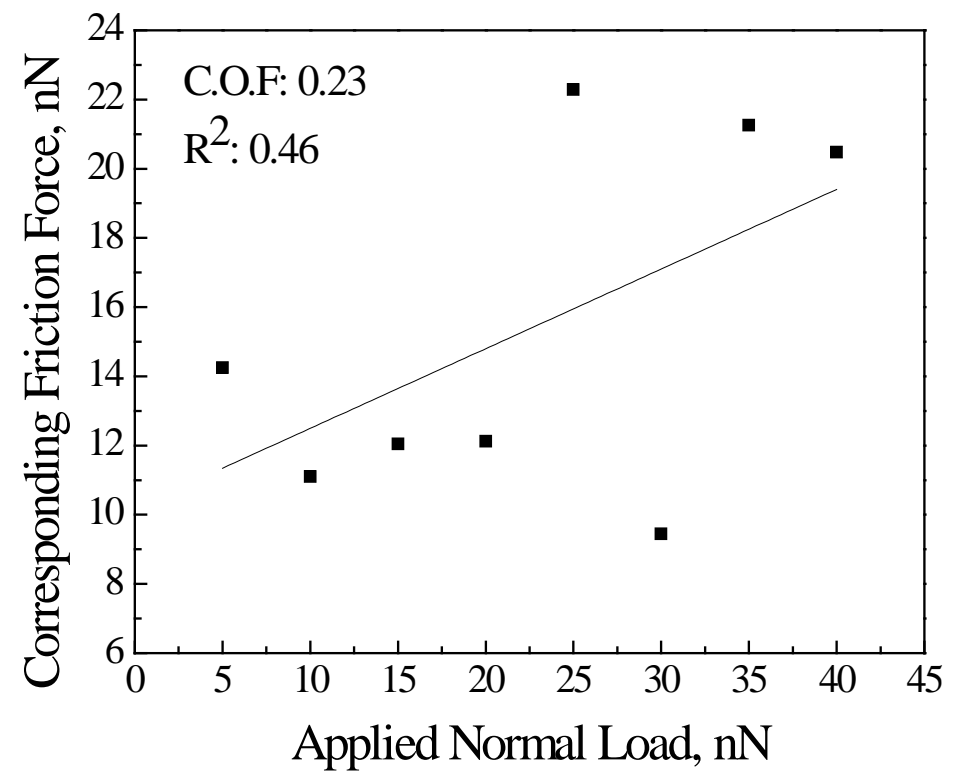

Figure 38 Friction Force vs Normal Load plot of oxidized T1 graphene sample 


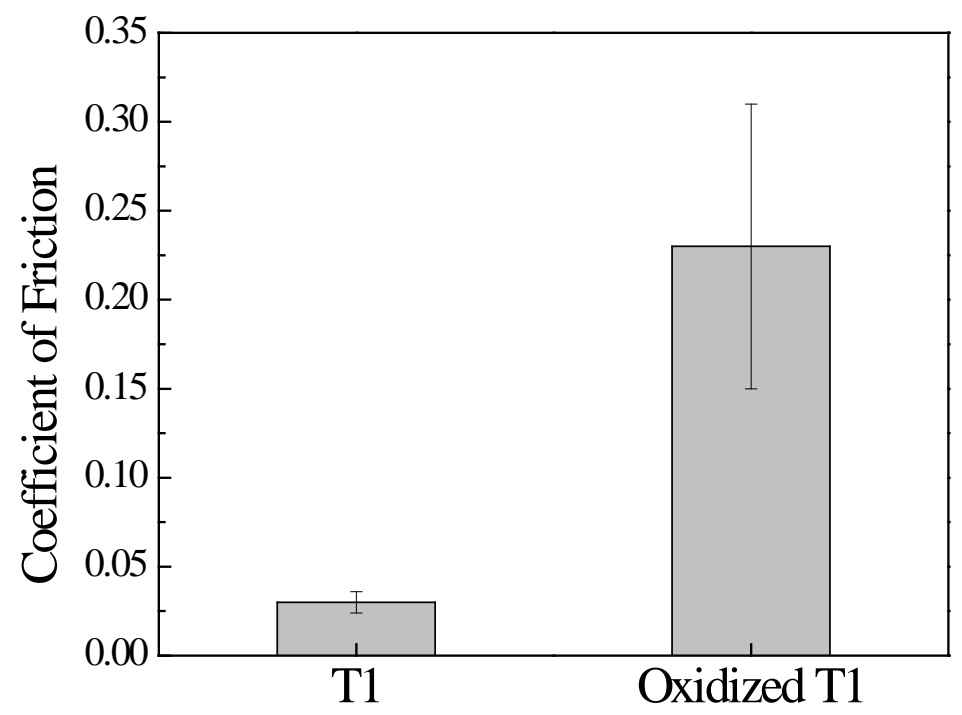

\section{Figure 39 Comparison of coefficient of friction between T1 and oxidized T1 graphene surfaces}

J. H. Ko, et al [40] determined the enhanced friction of chemically modified graphene with the increased out-of-plane elastic characteristics after chemical modification. They reported a 7fold increase in the nanoscale friction of oxidized graphene in comparison to the pristine graphene. Table 2 shows the increased carbonyl group produced edge defect concentration for the oxidized T1 graphene surface which correspond to the increase in its frictional values.

\subsection{Study of friction in different domains of an exfoliated graphene flake}

The friction forces on different domains of a T1 flake were determined to observe changes in friction of a single flake surface over different areas.

A $7 \mathrm{~nm}$ thickness $\mathrm{T} 1$ graphene flake was divided into 2 domains. Figure 41 shows the line profile across the two domains. The topography of the domain 1 indicates uniform thickness. 
However, the topography of the domain 2 suggests an increase in the thickness of upto $3 \mathrm{~nm}$ and high surface dislocations. These random variable thicknesses are a common feature of mechanically exfoliated flakes and may have an effect on the frictional characteristics of a graphene flake surface. Figure 42 showing the frictional plot of the flake shows a marked increase in the friction values between the 2 domains.

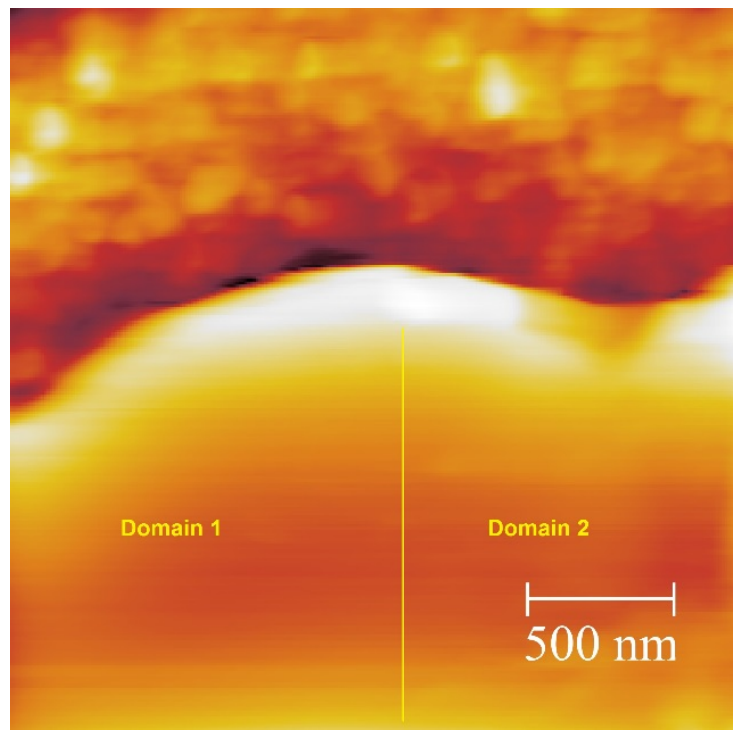

Figure 40 AFM topography of a T1 graphene flake divided into 2 domains (Z-Scale: 053.5nm)

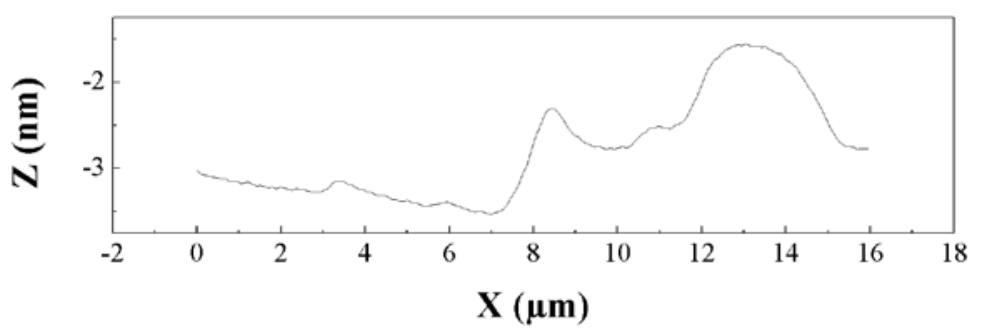

Figure 41 Line profile across the 2 domains 


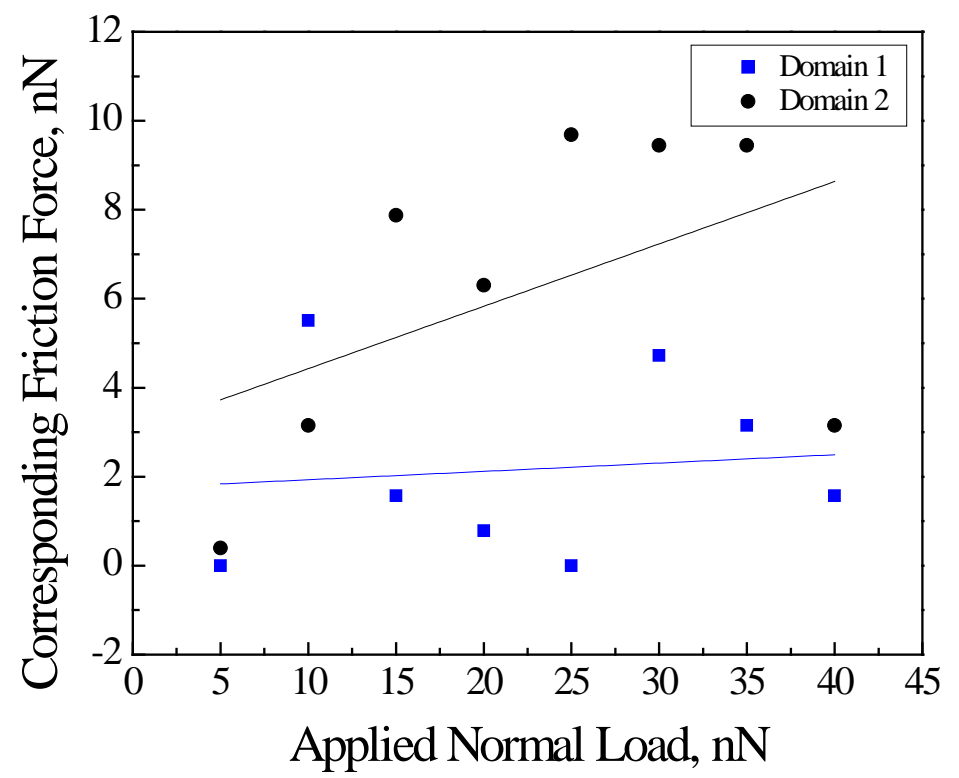

Figure 42 Friction Force vs Normal Load plot of a T1 graphene surface across 2 domains

\begin{tabular}{|cc|}
\hline Surface & C.O.F \\
Domain 1 & 0.02 \\
Domain 2 & 0.14 \\
\hline
\end{tabular}

\section{Table 3 Friction coefficient of different surfaces}

The increase in the friction force values for the same tip between the two domains can be attributed to the random variable thicknesses on the flg surface. The effect of the edge defects in the increase of frictional forces as seen in the previous section can be attributed to the increase of friction in the thickness varied domain 2 over relatively smooth domain 1 in the same T1 graphene flake surface. 


\section{FRICTION RESULTS IN LIQUIDS}

The application of graphene in different devices which include liquid components makes it important to study the friction between the sliding surfaces in the presence of different liquids. Friction results have been studied in different liquids to understand the effect of the environment in the interaction between a T3 graphene surface and a 'single' asperity Si tip. The T3 graphene surface well adhered to the lower roughness $\mathrm{SiC}$ substrate was chosen due to its uniformity on the substrate which makes it ideal to coat with liquids and obtain different areas with similar conditions to minimize measurement variability. To coat the graphene surface with a thin layer of liquid, all liquid solutions in the study have been spin coated onto the graphene surface at speeds of 2500 rpm for 60 seconds to obtain thin layers of liquids. $0.25 \mu$ of liquid was dispensed for each coating unless otherwise mentioned.

In order to check the influence of adsorbed water in ambient conditions, a thin layer of deionized water was coated on the graphene surface. Figure 43 shows the friction plot of graphene with a thin layer of DI water on its surface. 


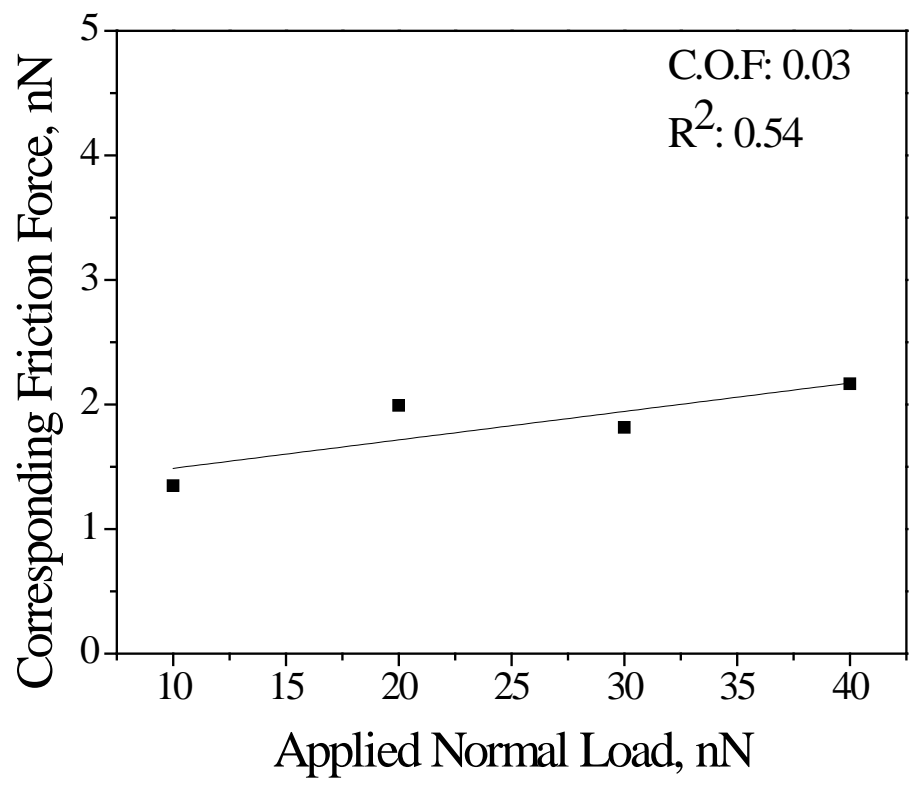

Figure 43 Friction Force vs Normal Load plot of T3 graphene in deionized water

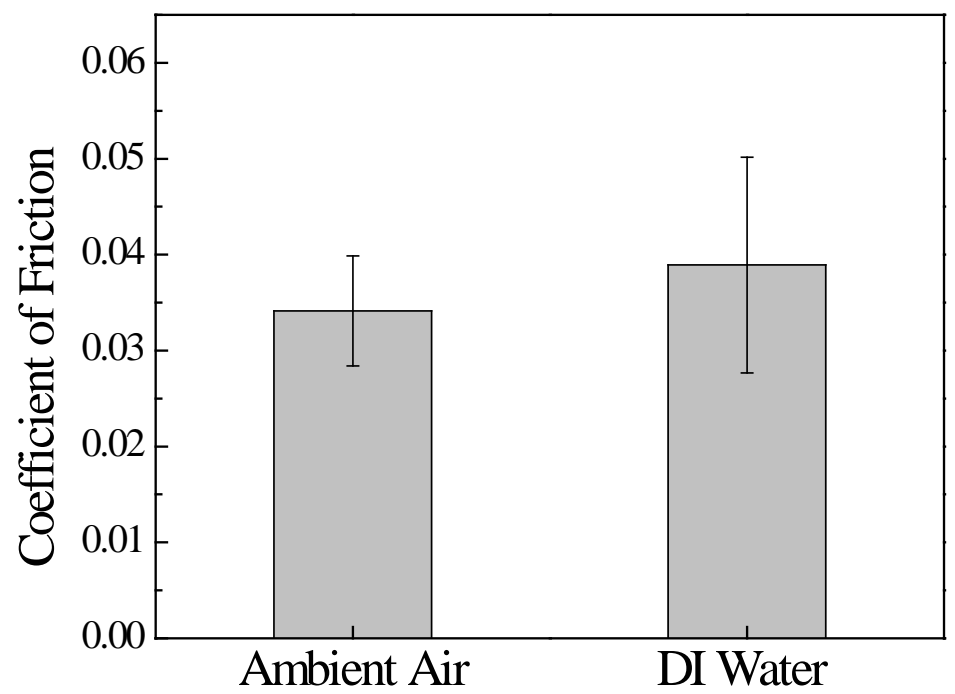

Figure 44 Comparison of coefficients of friction in ambient air and deionized water 
The magnitude of the coefficient of friction in both ambient air and with a de- ionized water surface remained relatively unchanged as shown in Figure 44. Sirghi [64] studied the effect of the capillary condensed water on the dynamic friction force at nano-asperity contacts on a Si surface. They observed the occurrence of friction at no or very small load and attributed friction to the process of dragging the capillary condensed water by the tip-sample contact. They also measured the frictional forces by hydrophobic and hydrophilic AFM tips on a hydrophilic Si surface at different humidities. These measurements showed a weak effect of humidity on friction for the hydrophobic tip and a strong and complex effect for the hydrophilic tip. Thus, moisture present in the atmosphere causes the hydrophilic Si AFM tip used in our study to have the same effect as that of the thin layer of water at the interface of the tip-graphene surface as evidenced by the comparison of the coefficients of friction in ambient air and deionized water.

\subsection{Friction in Ionic Liquids}

Ionic liquids have drawn considerable research interest for their role in energy storage devices, organic electronics, nano -tribology and for their chemical tenability and versatility [65]. They are considered as lubricants for NEMS/MEMS devices due to their excellent thermal and electrical conductivity. The integration of graphene into these devices would require the understanding of the modification of the interaction of the graphene surface when in contact with such ionic liquids. We study the interaction of graphene surface with the Si tip in the presence of two ionic liquids iodine and 1-butyl-3-methylimidazolium hexafluorophosphate (BMIM-PF 6$)$ solutions. 
The room temperature ionic liquids used in the study are Iodine solution (KI and Iodine in water) with a density of $0.7 \mathrm{gm} / \mathrm{ml}$ and 1-butyl-3-methylimidazolium hexafluorophosphate $\left(\mathrm{BMIM}-\mathrm{PF}_{6}\right)$ with a density of $1.37 \mathrm{gm} / \mathrm{ml}$. The solutions of the liquids $0.0995-0.1005 \mathrm{~N} \mathrm{KI}$ in water and 0.5\% (v/v) BMIM-PF 6 in isopropanol were coated onto the T3 graphene surface.

The friction plot for the sliding AFM tip on the T3 graphene surface in lubricating ionic liquids iodine and BMIM $\mathrm{PF}_{6}$ systems are shown in Figure 45 and 46 respectively.

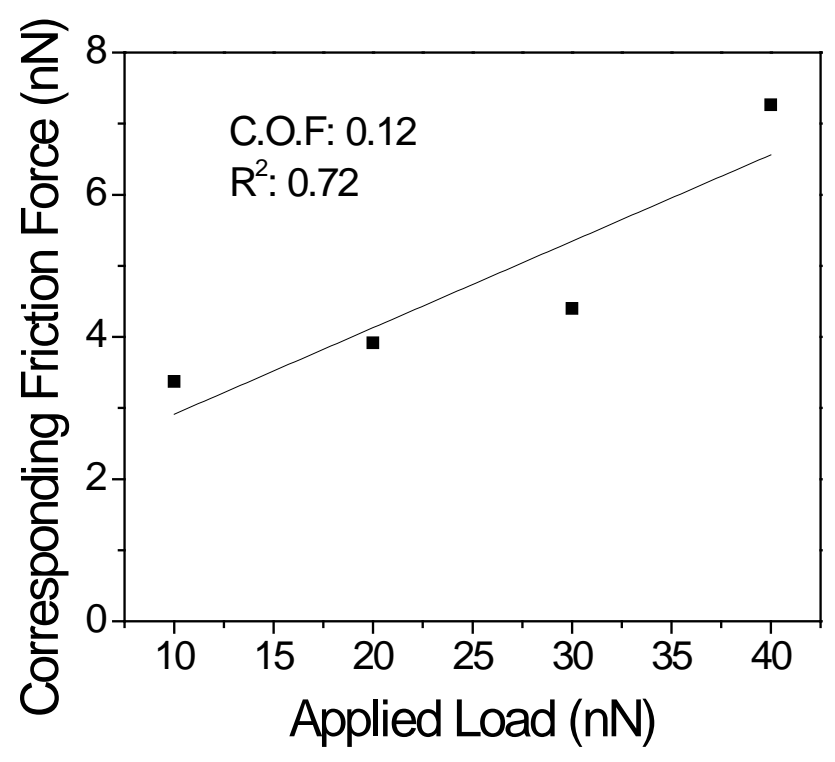

Figure 45 Friction Force vs Normal Load plot of T3 graphene in iodine lubricated system 


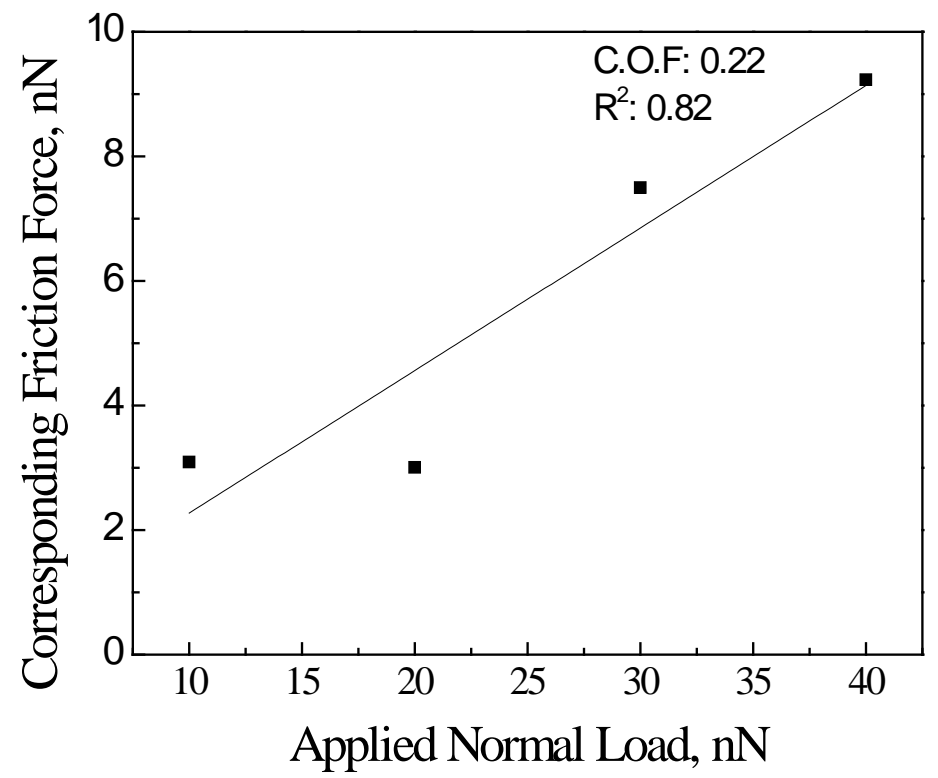

Figure 46 Friction Force vs Normal Load plot of T3 graphene in BMIM PF 6 lubricated system

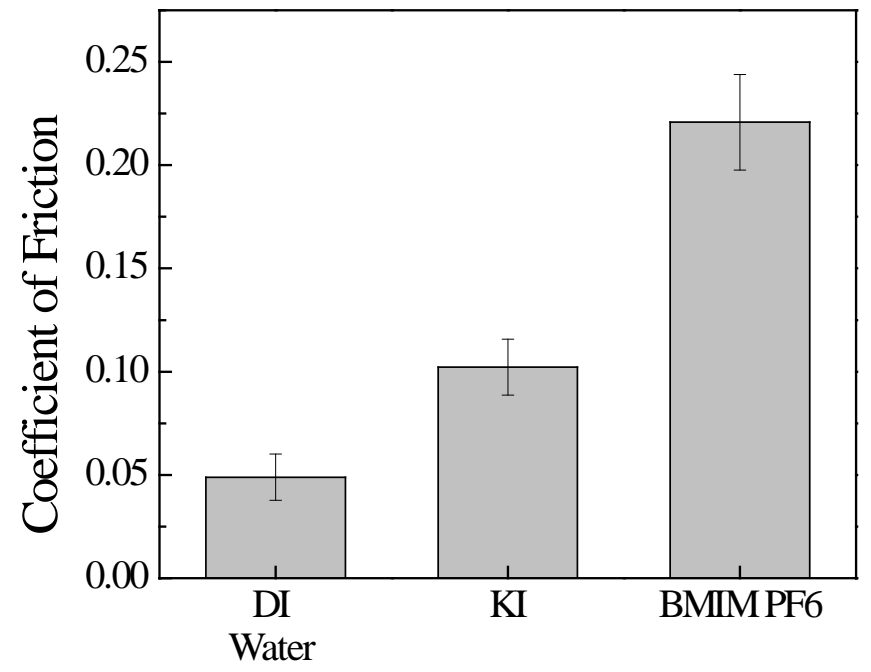

Figure 47 Comparison of coefficient of friction for different ionic liquid lubrication systems

We observe higher frictional forces in comparison to those obtained in air/water. In ionic liquids it is believed that aggregate formation in solvents that are less polar compared to water aids in minimizing the charge density (charge delocalization) within the ions [66] [67]. Also since 
no thermal treatment procedure was performed on the lubricant, its chemical bonding to the substrate is questionable. The lubricant molecules are less likely attached to the graphene surface and would tend to attract each other instead, such that dewetting is more likely [67]. Dewetting, the process that occurs at the solid- liquid interface is the rupture of the thin liquid film on the substrate and the formation of droplets causes the interaction of the lubricant to the substrate to be irregular as a result. Atmospheric water and lubricant molecules are more likely to form a meniscus as the tip approaches the surface which increase the friction forces on the sliding AFM.

XPS characterization of the liquid coated T3 graphene surfaces show the well coated surfaces.

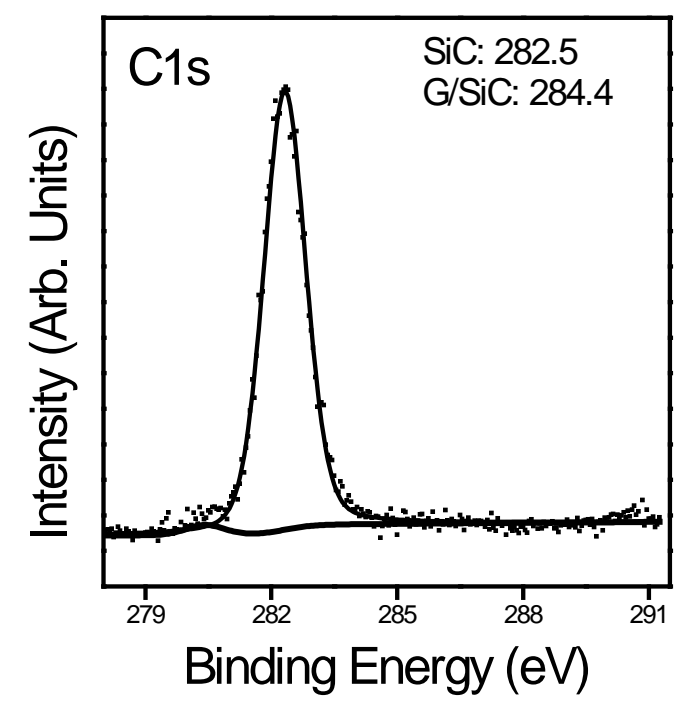

Figure 48 C1s Spectra of Iodine solution coated T3 graphene surface

Deconvolution of the C1s spectra yielded three peaks with different binding energy values representing C-C or graphene at $\sim 284.5 \mathrm{eV}$ along with the underlying substrate $\mathrm{SiC}$ at $\sim 282.5 \mathrm{eV}$. It also showed significant reduction of the oxygen defects. 
The XPS survey spectra of the BMIM PF6 lubricated system also showed a nearly completely covered surface with very small C, O and Si peaks composition. Other small F (1s), N (1s) and Na (KLL Auger) peaks were also observed.

Aqueous $\mathrm{NaCl}$ solution has been studied for its role in the nano- scale friction properties of graphene as a means to obtain the effect of its concentration. $\mathrm{NaCl}$ solutions with concentrations of $0.5 \%$ (wt. /vol.), 5\% (wt. /vol.), 7.5\% (wt. /vol.) and 10\% (wt. /vol.) were coated on the graphene surface and the friction plots were obtained as follows.

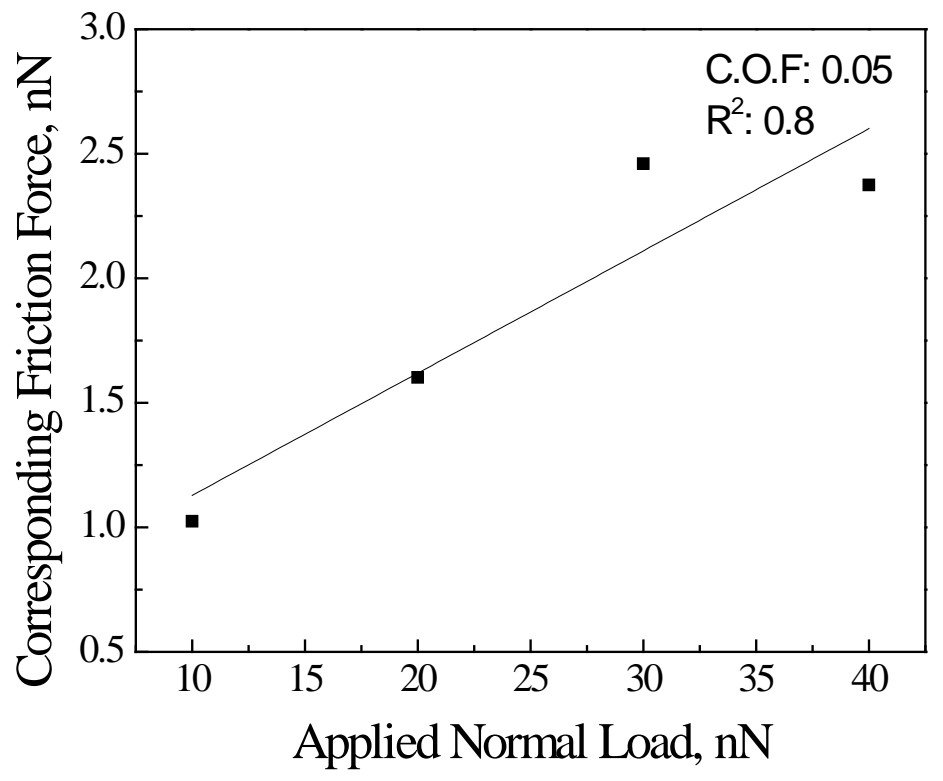

Figure 49 Friction Force vs Normal Load plot of T3 graphene in $0.5 \%$ (wt. /vol) NaCl system 


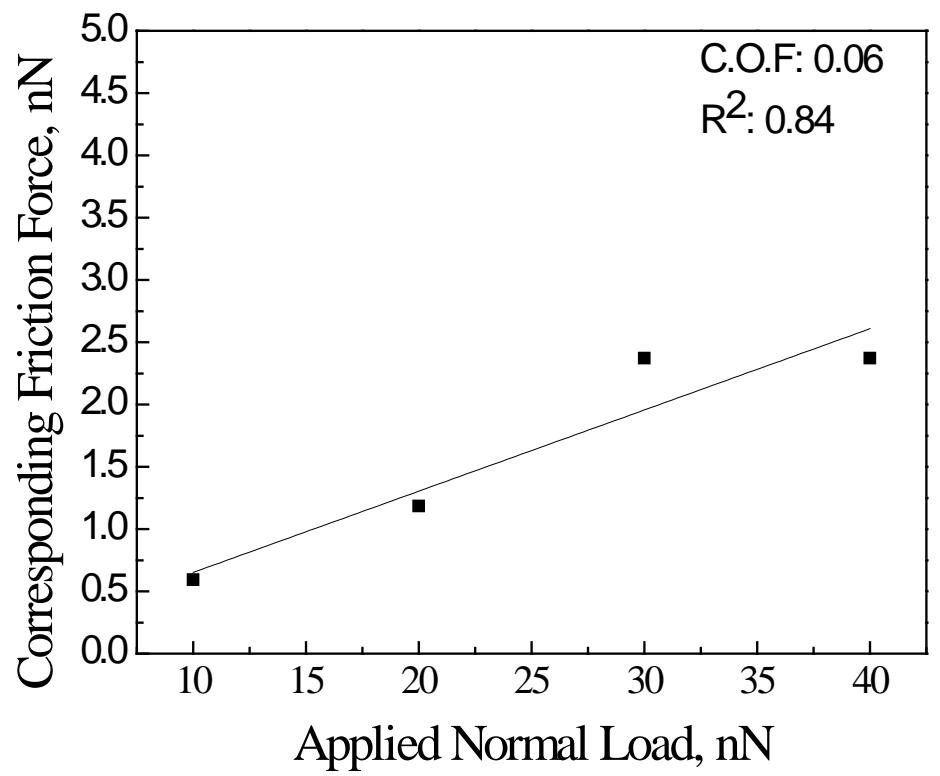

Figure 50 Friction Force vs Normal Load plot of T3 graphene in 5\% (wt. /vol) NaCl system

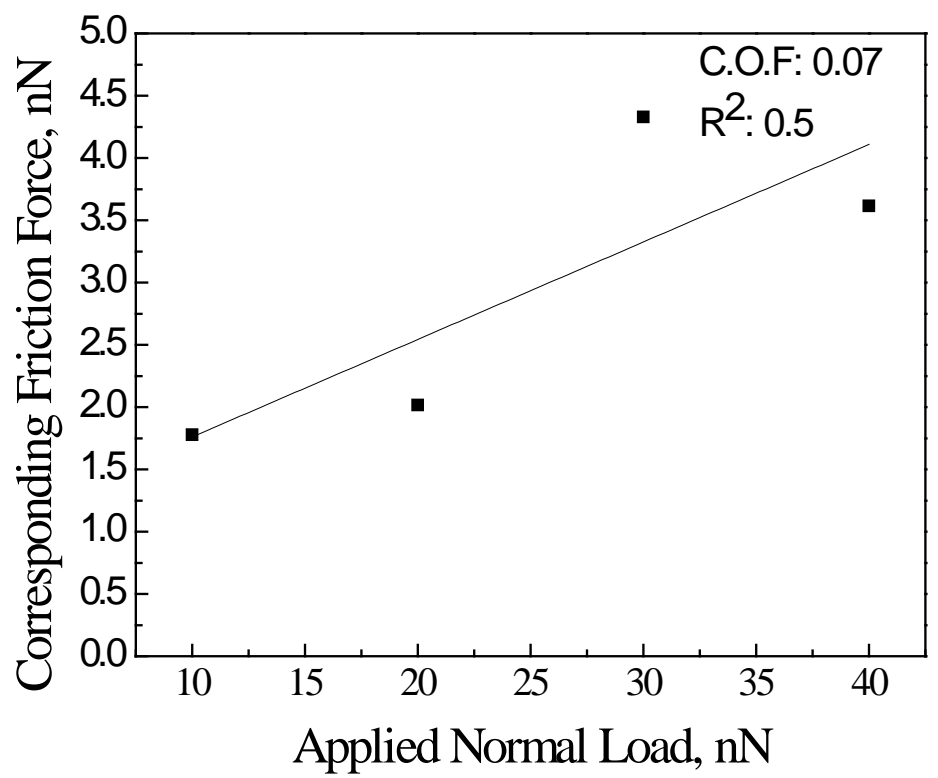

Figure 51 Friction Force vs Normal Load plot of T3 graphene in 7.5 \% (wt. /vol) NaCl system 


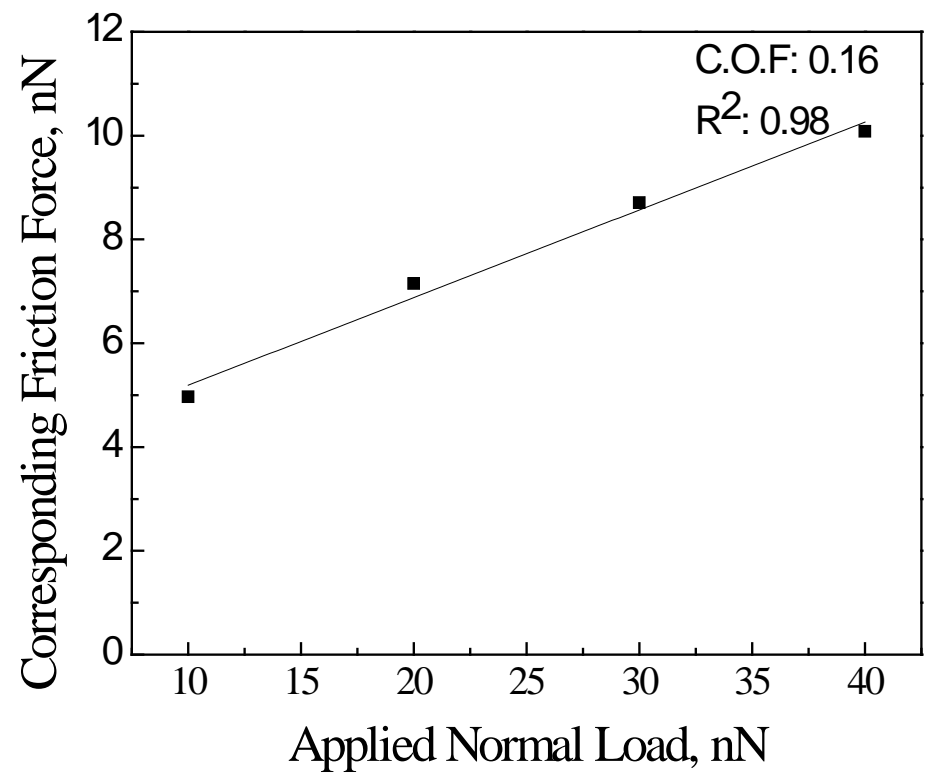

Figure 52 Friction Force vs Normal Load plot of T3 graphene in 10 \% (wt. /vol) NaCl system

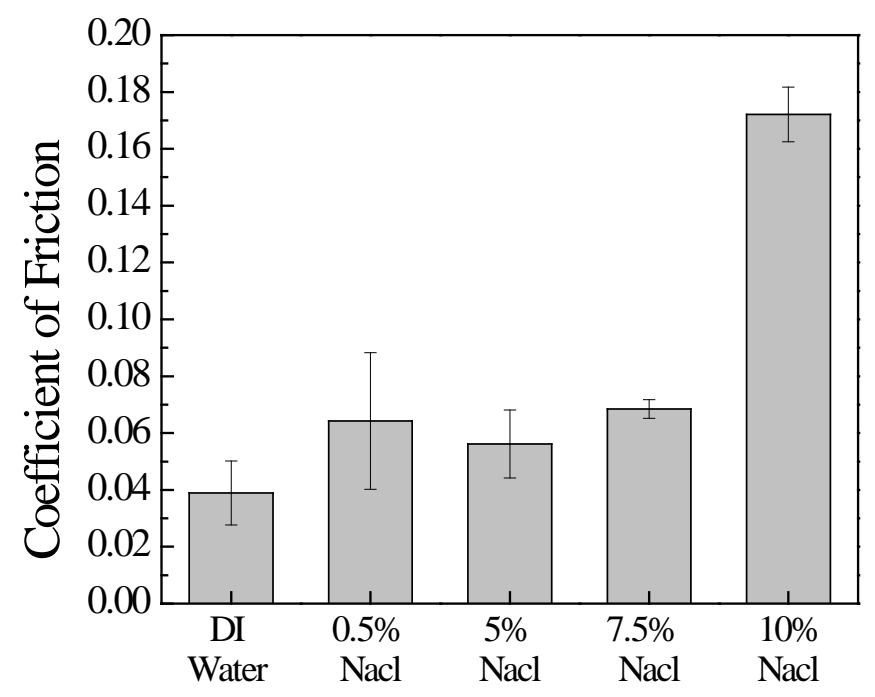

Figure 53 Comparison of coefficient of friction for $\mathrm{NaCl}$ solutions of different concentrations 

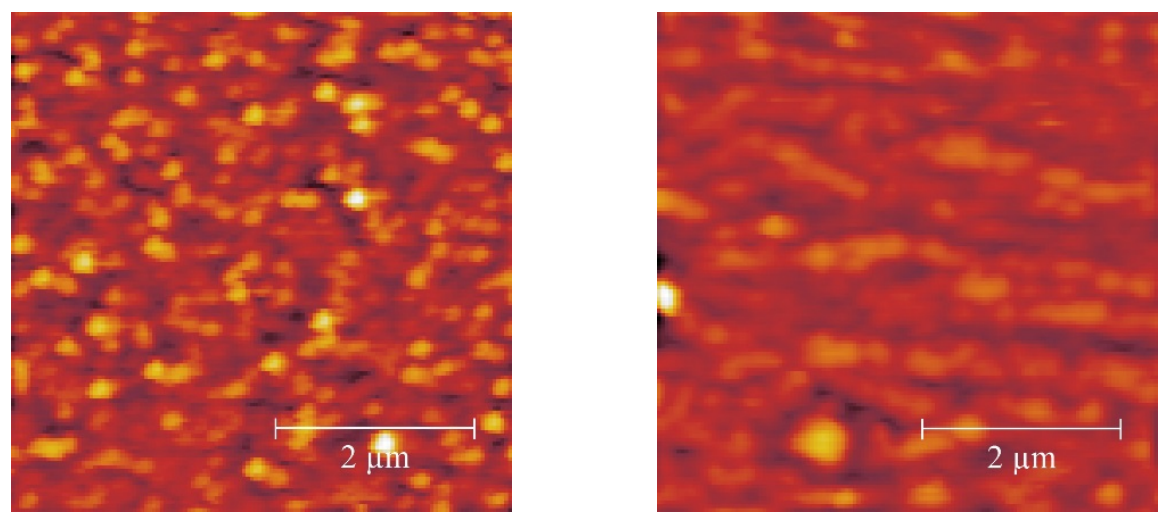

Figure 54 AFM topography of T3 graphene coated with 5\% (wt./vol) and $10 \%$ (wt./vol) NaCl solutions (Z- Scale: 0 - 8.3 nm (5\% (wt./vol)) and 0- 13.5 nm (10\% (wt./vol))).

Aggregates of increased sizes are observed in the topographic images obtained for the $10 \%$ (wt. /vol.) NaCl solution as shown in Figure 54. These aggregates could be a result of the increased concentration of the salts in the preparation of the solution. The size of these aggregates are important as the tip asperity interactions at these interfaces have high localized pressures resulting in increased local stress fields that can cause materials to shear as they encounter each other during sliding. Therefore effect of $\mathrm{NaCl}$ concentration directly influences the magnitude of the aggregate size and hence the frictional forces between the sliding AFM tip and the coated graphene surface.

\subsection{Friction in Oils}

Silicone oils are primarily used as lubricants because of their relatively high thermal stability and their lubricating properties.

In order to compare the friction properties in the presence of silicone oils of different viscosities, LFM was performed on graphene surfaces covered with silicone oils of viscosities 4.7 cP and $9.2 \mathrm{cP}$. 


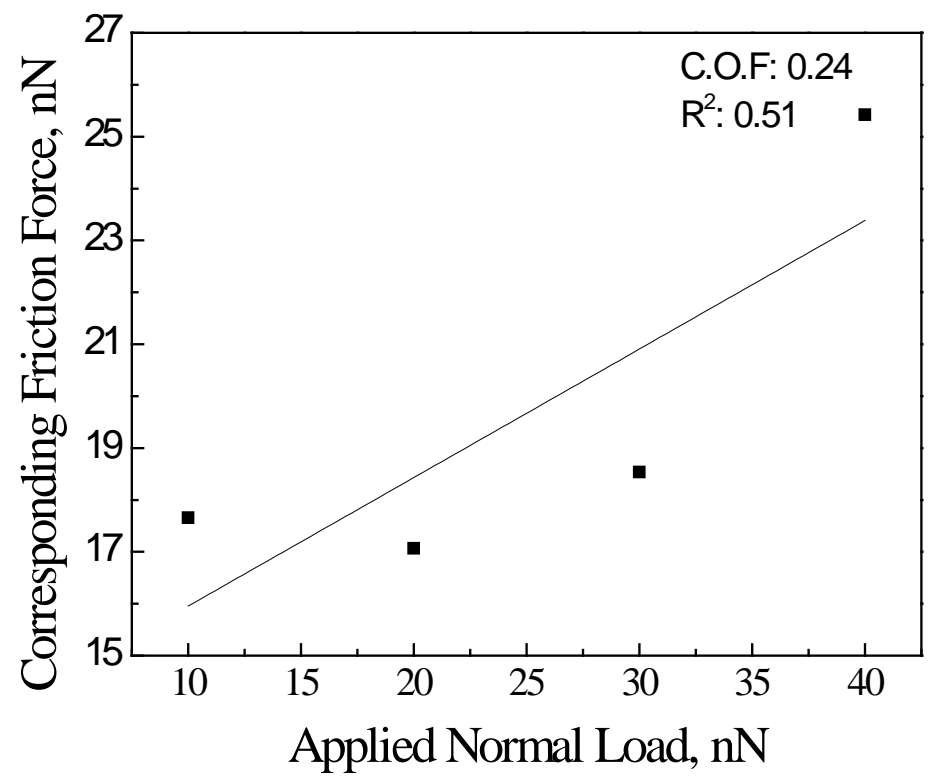

Figure 55 Friction Force vs Normal Load plot of T3 graphene in silicone oil (4.7 cP) lubricated system

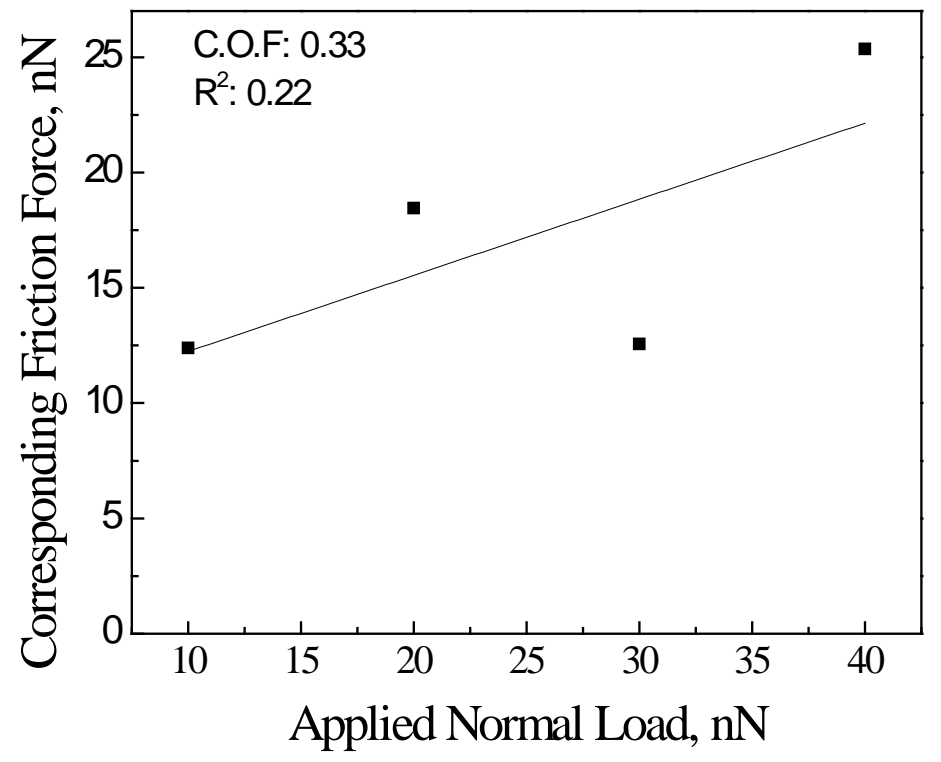

Figure 56 Friction Force vs Normal Load plot of T3 graphene in silicone oil $(9.2 \mathrm{cP})$ lubricated system 


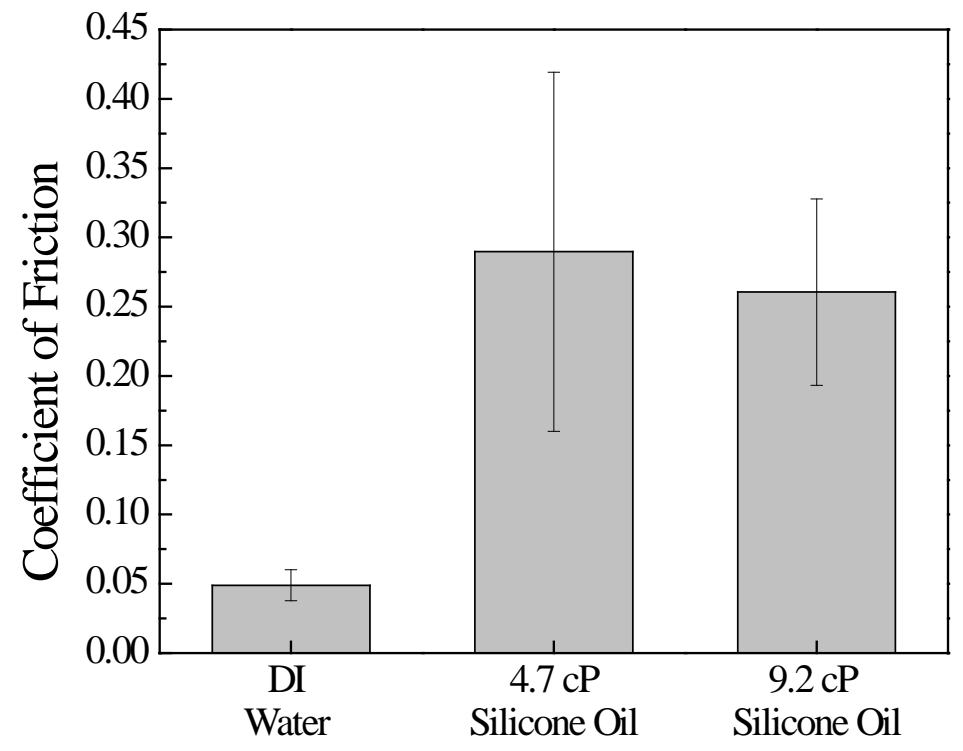

Figure 57 Comparison of coefficient of friction in water and silicone oil lubricated systems

We observe higher frictional forces for the silicone oil lubricated system in comparison to those obtained in air/water as shown in Figure 57. These higher values can be attributed to the viscous like boundary film being formed on the surface. The lateral chain-chain Van Der Waals interaction within the film increases the film stiffness thereby increasing the shear on the sliding tip. Lubricating molecules trapped between and around the contact region of the tip and the flg surface and a similar ordering of the non-polar lubricant within the immediate region of the tip was found to increase the frictional forces [14]. This was observed using XPS characterization where the surface of graphene was virtually found to be completely covered with silicone oil as can be seen in the C1s spectra shown in Figure 58. 


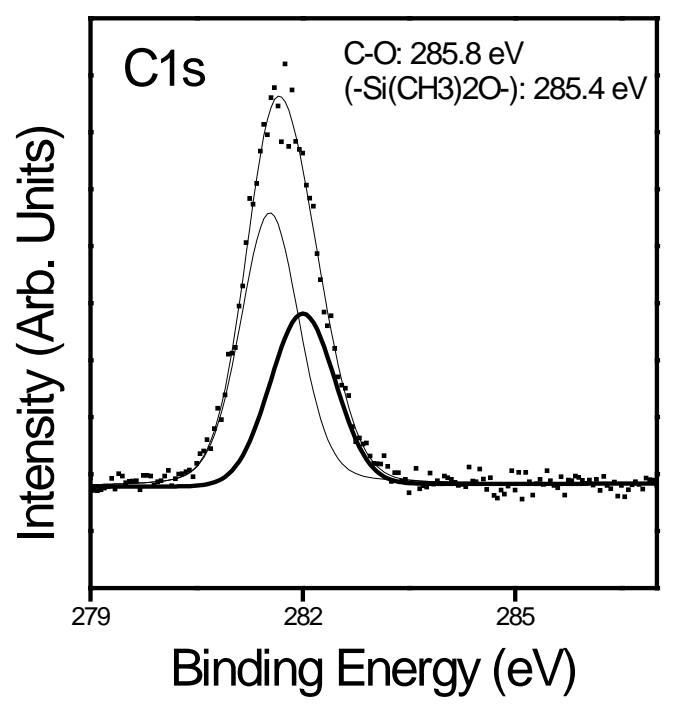

Figure 58 C1s Spectra of Silicone oil coated T3 graphene surface

Deconvolution of the C1s spectra yielded three peaks with different binding energy values representing $\mathrm{C}-\mathrm{O}$ at $\sim 285.8 \mathrm{eV}$ and $\left(-\mathrm{Si}\left(\mathrm{CH}_{3}\right) 2 \mathrm{O}-\right)$ at $\sim 285.4 \mathrm{eV}$. Other terminal groups on the silicon chain were found from the Si2s spectra. This oil surface creates an interlocking layer which effectively increases the frictional forces on the sliding AFM tip over the flg surface.

\subsection{Effect of Sliding Speed on Friction}

To further observe the role of the meniscus in dynamic friction and thereby the effect of scanning speed on the coefficient of friction, LFM was performed for varying speeds between 1 lnps -5 lnps or $5 \mu \mathrm{m} / \mathrm{s}$ to $25 \mu \mathrm{m} / \mathrm{s}$ on different lubricated systems. The results are shown in the following figures. 


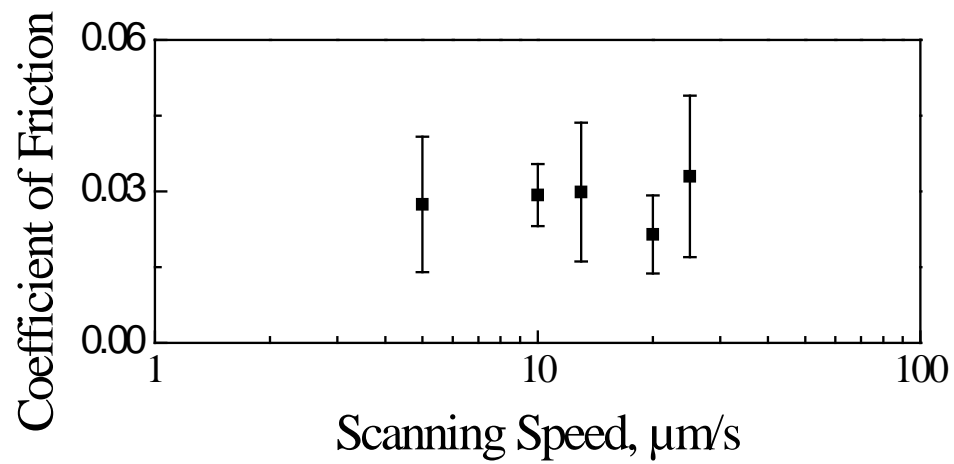

Figure 59 Effect of scanning rate on friction in DI water system

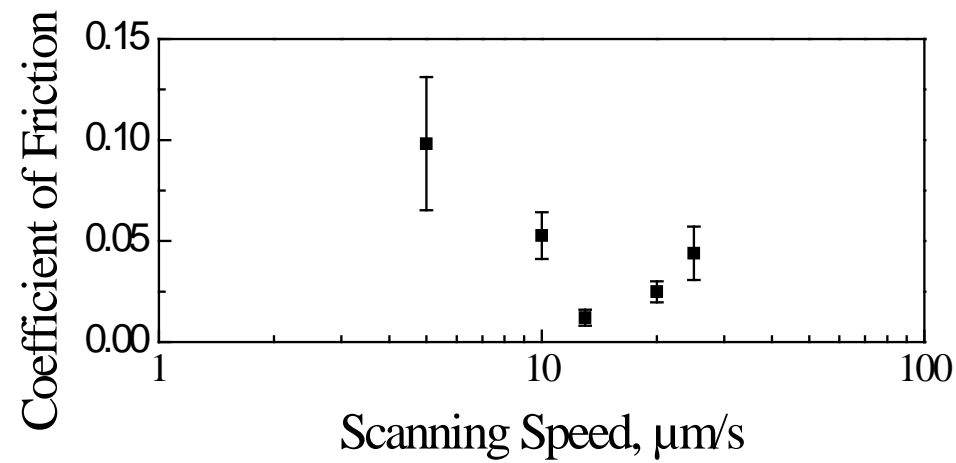

Figure 60 Effect of scanning rate on friction in $0.5 \%$ (wt. /vol) $\mathrm{NaCl}$ system

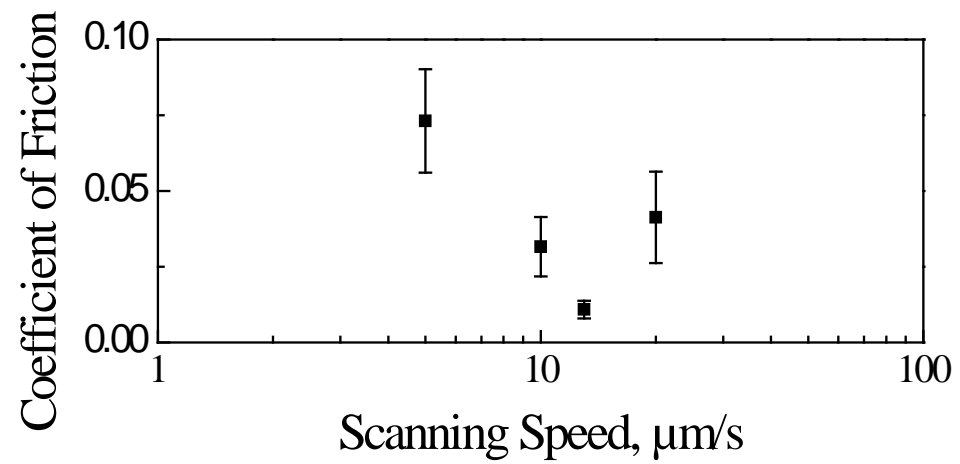

Figure 61 Effect of scanning rate on friction in $5 \%$ (wt. /vol) $\mathrm{NaCl}$ system 


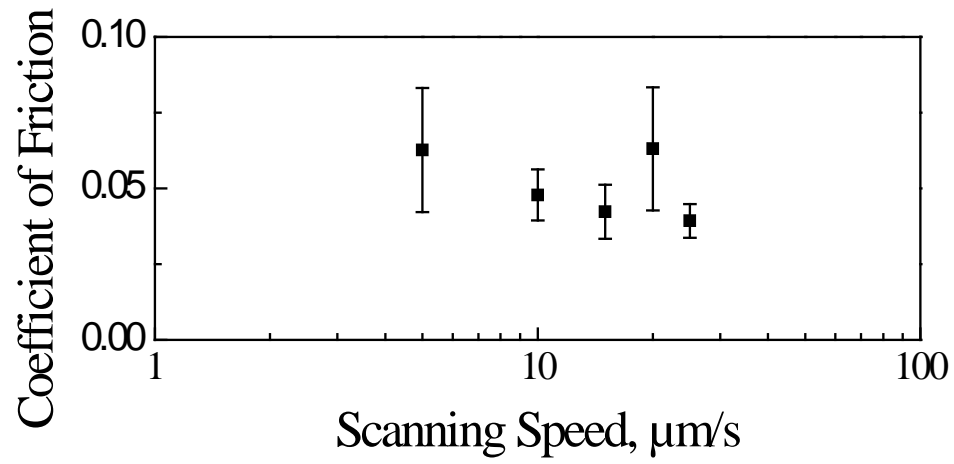

Figure 62 Effect of scanning rate on friction in $7.5 \%$ (wt. /vol) NaCl system

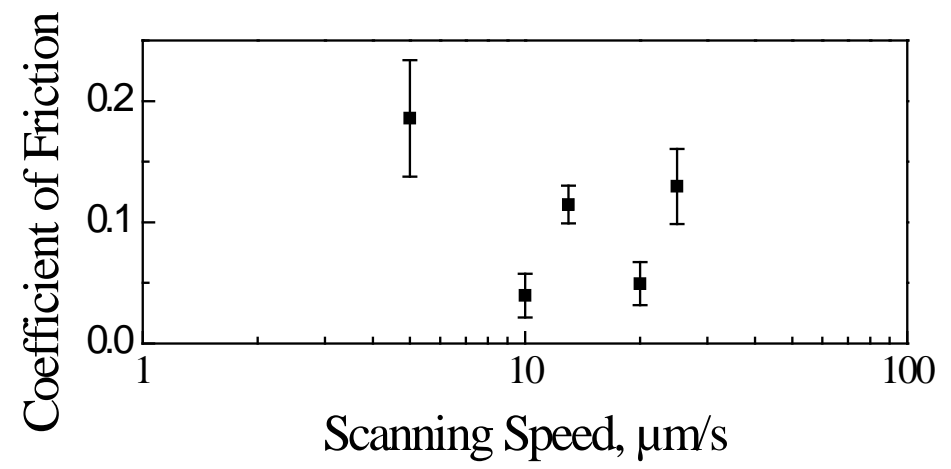

Figure 63 Effect of scanning rate on friction in $10 \%$ (wt. /vol) NaCl system

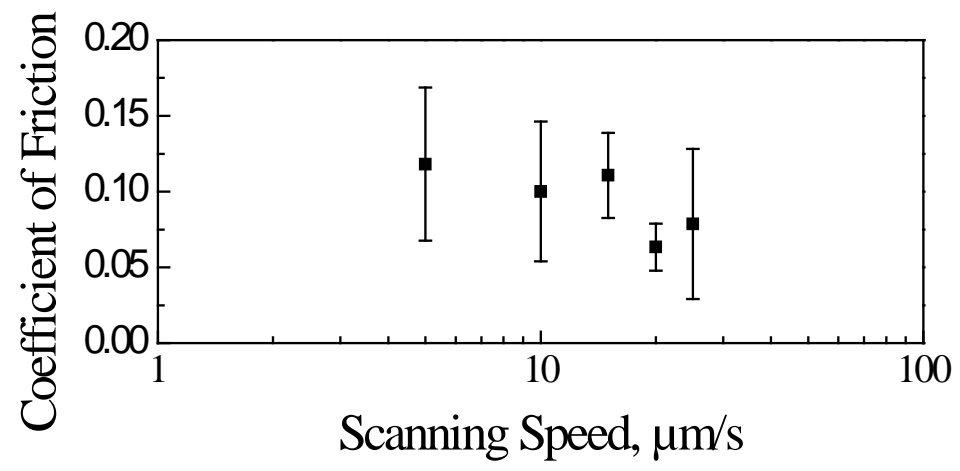

Figure 64 Effect of scanning rate on friction in Iodine lubricated system 


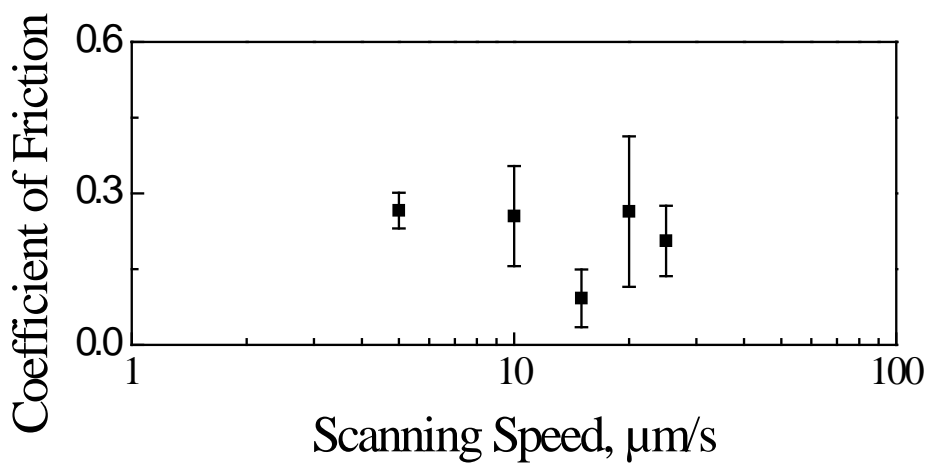

Figure 65 Effect of scanning rate on friction in BMIM PF 6 lubricated system

The results indicate that the nanoscale frictional forces reduce with increased scan rates for the lubricated systems. With the increase of scanning rate, increase of kinetic energy has an effect on the meniscus formation make it difficult to form completely which could have an effect in the reduction.

However, a Stribeck-like curve was observed for the silicone oil lubricated system.

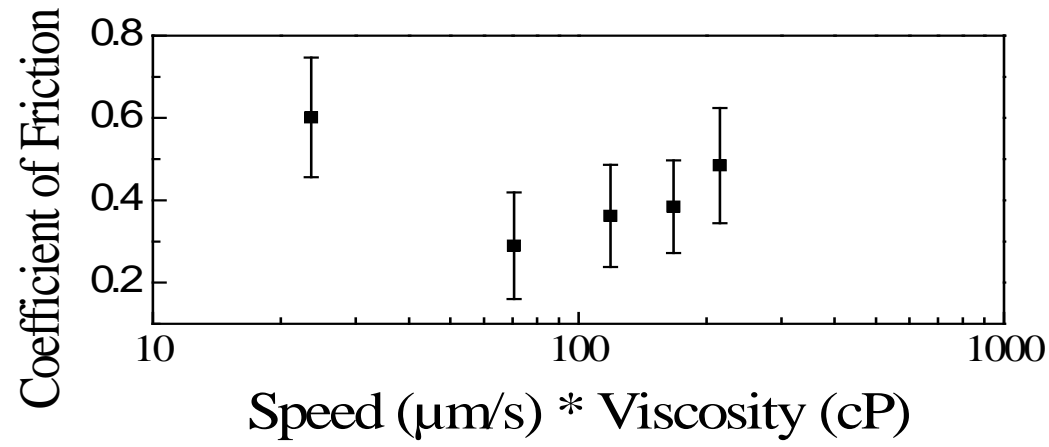

Figure 66 Stribeck-like plot for T3 graphene in silicone oil (4.7 cP) lubricated system 


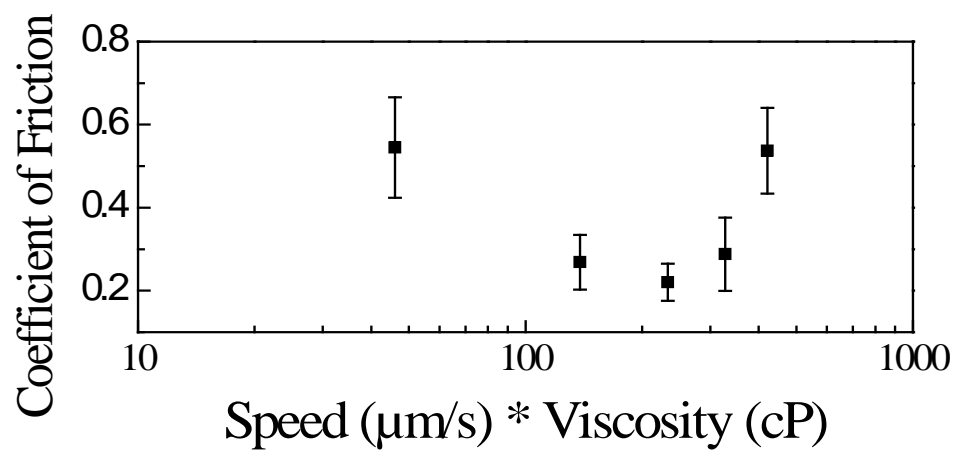

Figure 67 Stribeck-like plot for T3 graphene in silicone oil $(9.2 \mathrm{cP})$ lubricated system

Stribeck curves show the transition of the lubricating conditions of the sliding surfaces from boundary lubrication to mixed lubrication and the hydrodynamic lubrication regimes [68].

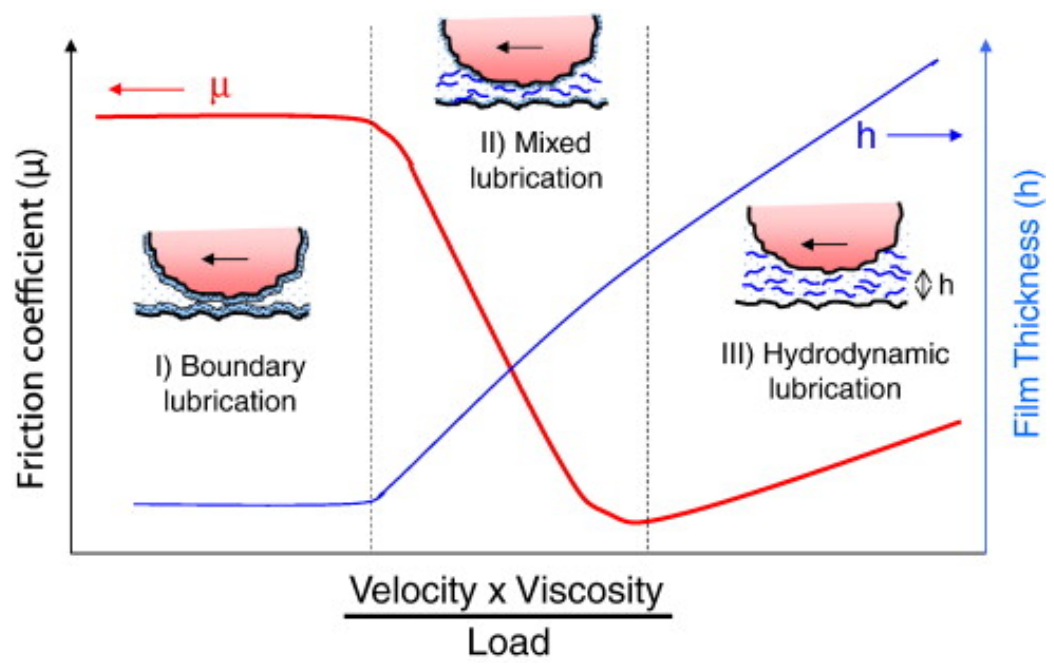

Figure 68 Schematic showing boundary, mixed, and hydrodynamic lubrication regimes [68] 
In boundary lubrication, an absorbed layer or tribo-chemical reaction layer lies between surfaces in the real contact area to control the adhesion between solid interfaces and to reduce the shearing resistance; this in turn decreases the coefficient of friction relative to dry friction. In mixed film lubrication, the coefficient of friction decreases because the fluid film of small shearing resistance bears a portion of the load, and the real contact area are decreased. In hydrodynamic lubrication, the coefficient of friction is at its minimum because the real contact area disappears owing to the fluid film bearing the entire load, and the frictional force is due to only the viscous resistance of the fluid. However, when the fluid viscosity and sliding speed further increase the bearing characteristic number, the coefficient of friction rises owing to the increase in viscous resistance [69]. This correlates to the increase in friction for the silicone oil lubricated system where the liquid layer completely covering the surface increases the friction on the sliding tip. 


\section{CONCLUSIONS}

We have studied the nanoscale adhesion and frictional properties of flg synthesized by topdown and bottom-up methods. Both adhesion and friction have been found to be dependent on the method of synthesis and deposition. The adhesion force was found to depend upon the interfacial forces between the graphene and the underlying substrate and also on the oxygen defect composition of the flg surfaces. The liquid phase exfoliation produced graphene surface (T1) and reduced graphene oxide surface (T2) deposited on silicon oxide substrates were found to have higher values of adhesion in comparison to the halogen based plasma etched graphene on silicon carbide substrate (T3) due to the sample substrate interfacial forces. The defect composition was found to be lowest in the case of T2 and nearly similar for T1 and T3 graphene surfaces. These defects were found to affect the adhesion forces between the single asperity silicon AFM tip and the flg sample. We also found a decreasing pattern of adhesion forces along the central part of the flake towards the edge.

The frictional forces between the Si AFM tip and the flg surfaces were found to be dominated by the underlying substrate as well. Rougher substrate conformation by the graphene surface increased the frictional forces on the sliding tip in the case of T1 and T2 graphene surfaces. In all the systems, the coefficient of friction values of the different graphene surfaces were found to be lower than that of their underlying substrates making them ideal for surface coatings in NEMS/MEMS based devices to reduce friction. It was also found that plasma oxidation of the graphene surface induced oxygen defects and increased the adhesion and frictional forces on the flg. 
The frictional forces in the presence of liquids showed interesting results wherein the effect of a thin water surface did not affect nanoscale friction and had the same effect of moisture present in the ambient atmospheric conditions. Ionic liquids iodine and BMIM-PF 6 had an increasing effect on the nanoscale friction due to aggregation of the weakly bonded lubricating molecules. Silicone oils were also found to have an increasing effect on the friction due to the increased molecular interactions around the tip at the lubricating interface. The effect of sliding speed on friction in the presence of liquids was studied and Stribeck like curves were obtained for the silicone oil lubricated systems. The liquid meniscus formed between the sliding AFM tip and the graphene surface in water was found to have the lowest effect on the frictional forces in comparison to other lubricating ionic liquids and silicone oils.

Future work in this direction would be to further study the effects of functionalized tips in the adhesion and frictional properties of the flg surfaces. This functionalization may explain the effect of the meniscus formation in both air and liquids which plays a significant role in adhesion and friction at the nanoscale. Further, the bonding between the lubricants to the graphene surface need to be studied in depth to understand its effect on the frictional characteristics of lubricant based systems. 


\section{REFERENCES}

[1] K. S. Novoselov, A. K. Geim, S. V. Morozov, D. Jiang, Y. Zhang, S. V. Dubonos, I. V. Grigorieva and A. A. Firsov, "Electric field effect in atomically thin carbon films," Science, vol. 306, p. 666-669, 2004.

[2] A. K. Geim and K. S. Novoselov, "The rise of graphene," Nature Materials, vol. 6, pp. 183191, 2007.

[3] D. Berman, A. Erdemir and A. V. Sumant, "Graphene: a new emerging lubricant," Materials Today, vol. 17, pp. 31-42, 2014.

[4] M. J. Allen, V. C. Tung and R. B. Kaner, "Honeycomb carbon: a review of graphene.," Chemical Reviews, vol. 110, pp. 132-145, 2010.

[5] K. P. Loh, Q. Bao, P. K. Ang and J. Yang, "The chemistry of graphene," Journal of Materials Chemistry, vol. 20, pp. 2277- 2289, 2010.

[6] H. W.J., O. Park and B. Chun, "Foldable graphene electronic circuits based on paper substrates.," Advanced Materials, vol. 25, pp. 4729- 4734, 2013.

[7] X. Zhao, C. M. Hayner, M. C. Kung and H. H. Kung, "In-Plane Vacancy-Enabled HighPower Si-Graphene Composite Electrode for Lithium-Ion Batteries," Advanced Energy Materials, vol. 1, pp. 1079- 1084, 2011. 
[8] D. Prasai, J. C. Tuberquia, R. R. Harl, G. K. Jennings and K. I. Bolotin, "Graphene: Corrosion-Inhibiting Coating," ACS Nano, vol. 6, p. 1102, 2012.

[9] A. A. Balandin, S. Ghosh, W. Bao, I. Calizo, D. Teweldebrhan, F. Miao and C. N. Lau, "Superior Thermal Conductivity of Single Layer - Graphene," Nano Letters, vol. 8, pp. 902907, 2008.

[10] D. Berman, A. Erdemir and A. V. Sumant, "Few layer graphene to reduce wear and friction on sliding steel surfaces," Carbon, vol. 54, pp. 454- 459, 2013.

[11] G. Ren, Z. Zhang, X. Zhu, B. Ge, F. Guo, X. Men and W. Liu, "Influence of functional graphene as filler on the tribological behaviors of Nomex fabric/phenolic composite," Composites Part A: Applied Science and Manufacturing, vol. 49, pp. 157-164, 2013.

[12] K. J. Huang, L. Wang, J. Li and Y. M. Liu, "Electrochemical sensing based on layered MoS2graphene composites," Sensors and Actuators B, vol. 178, pp. 671- 677, 2013.

[13] J. Lin, L. Wang and G. Chen, "Modification of Graphene Platelets and Their Tribological Properties as a Lubricant Additive.," Tribology Letters, vol. 41, pp. 209- 215, 2011.

[14] B. Robinson, N. Kay and O. Kolosov, "Adhesion and Dynamic Nanotribology of Graphene in polar and non- polar liquid environments studied with ultrasonic force microscopy," NSTI Nanotech, vol. 1, pp. 820- 827, 2012. 
[15] B. Stegemann, H. Backhaus, E. Santner and H. Kloss, "Spherical AFM probes for adhesion Force Measurements on Metal Single Crystals," 2007. [Online]. Available: http://www.formatex.org/microscopy3/pdf/pp820-827.pdf. [Accessed 30 June 2015].

[16] H. Bai, C. Li, X. Wang and S. G, "On the Gelation of Graphene Oxide," Physical Chemistry, vol. 115, p. 5545-5551, 2011.

[17] S. Z. Butler, S. M. Hollen, L. Cao, Y. Cui, J. A. Gupta, H. R. Gutiérrez, T. F. Heinz, S. S. Hong, J. Huang, A. F. Ismach, E. Johnston-Halperin, M. Kuno, V. V. Plashnitsa, R. D. Robinson and R. S. Ruo, "Progress, Challenges, and Opportunities in Two-Dimensional," ACS Nano, vol. 7, p. 2898-2926, 2013.

[18] D. R. Cooper, B. D’Anjou, N. Ghattamaneni, B. Harack, M. Hilke, A. Horth, N. Majlis, M. Massicotte, L. Vandsburger, E. Whiteway and V. Yu, "Experimental Review of Graphene," ISRN Condensed Matter Physics, vol. 2012, pp. 1-56, 2012.

[19] D. R. Dreyer, P. R. S. Ruoff and P. C. W. Bielawski, "From Conception to Realization: An Historial Account of Graphene and Some Perspectives for Its Future," Angewandte Chemie International Edition, vol. 49, p. 9336-9344, 2010.

[20] H. P. Boehm, R. Setton and E. Stumpp, "Nomenclature and terminology of graphite intercalation compounds," Synthetic Metals, vol. 11, p. 363-371, 1985.

[21] H. Boehm, R. Setton and E. Stumpp, "Nomenclature and terminology of graphite intercalation compounds (IUPAC Recommendations 1994)," Pure and Applied Chemistry, vol. 66, pp. 1893-1901, 1994. 
[22] E. Fitzer, K. Kochling, H. P. Boehm and H. Marsh, "Recommended terminology for the description of carbon as a solid (IUPAC Recommendations 1995)," Pure and Applied Chemistry, vol. 67, pp. 473-506, 1995.

[23] K. S. Novoselov, A. K. Geim, S. V. Morozov, D. Jiang, Y. Zhang, S. V. Dubonos, I. V. Grigorieva and A. A. Firsov, "Electric Field Effect in Atomically Thin Carbon Films," Science, vol. 306, pp. 666-669, 2004.

[24] S. V. Morozov, K. S. Novoselov, M. I. Katsnelson, S. F., D. C. Elias, J. A. Jaszczak and A. K. Geim, "Giant Intrinsic Carrier Mobilities in Graphene and Its Bilayer," Physical Review Letters, vol. 100, pp. 016602-016605, 2008.

[25] K. I. Bolotin, K. J. Sikes, Z. Jiang, M. Klima, G. Fudenberg, J. Hone, P. Kim and H. L. Stormer, "Ultrahigh electron mobility in suspended graphene," Solid State Communications, vol. 141, pp. 351-355, 2008.

[26] A. B. Alexander, "Thermal properties of graphene and nanostructured carbon materials," Nature Materials, vol. 10, pp. 569-581, 2011.

[27] W. F. I., M. T. D., d. Z. A. M. van and L. M. P., "Mechanical properties of suspended graphene sheets," American Vacuum Society, vol. 25, pp. 2558-2561, 2007.

[28] C. Lee, Q. Li, W. Kalb, X.-Z. Liu, H. Berger, R. W. Carpick and J. Hone, "Frictional Characteristics of Atomically Thin Sheets," Science, vol. 328, pp. 76-80, 2010. 
[29] S. Paolo and A. Ciesielski, "Graphene via sonication assisted liquid-phase," Chemical Society Reviews, vol. 43, pp. 381-398, 2014.

[30] V. Singh, D. Joung, L. Zhai, S. Das, S. Khondaker and S. Seal, "Graphene based Materials: Past, Present and Future," Progress in Material Science, vol. 56, pp. 1178-1271, 2011.

[31] M.-S. Won, O. V. Penkov and D.-E. Kim, "Durability and Degradation Mechanism of Graphene Coatings Deposited on Cu Substrates under Dry Contact Sliding," Carbon, vol. 54, pp. 472-481, 2013.

[32] J. Hass, W. A. d. Heer and E. H. Conrad, "The Growth and Morphology of Epitaxial Multilayer Graphene," Journal of Physics Condensed Matter, vol. 20, p. 323202, 2008.

[33] F. Varchon, R. Feng, J. Hass, X. Li and N. N. B., "Electronic Structure of Epitaxial Graphene Layers on SiC: Effect of the Substrate," Physical Review Letters, vol. 99, p. 126805, 2007.

[34] J. Penuelas, A. Ouerghi, D. Lucot, C. David, J. Gierak, E. H. Szwarckopf and A. C. Vignolle, "Surface Morphology and Characterization of thin Graphene Films on SiC Vicinal Substrate," Physical Review B, vol. 79, 2009.

[35] X.-Z. Liu, Q. Li, P. Egberts and R. W. Carpick, "Nanoscale Adhesive Properties of Graphene: The Effect of Sliding History," Advanced Materials Interfaces, vol. 1, 2014.

[36] Y. J. Shin, R. Stromberg, R. Nay, H. Huang, A. T. Wee, H. Yang and C. S. Bhatia, "Frictional characteristics of exfoliated and epitaxial graphene," Carbon, vol. 49, pp. 4070- 4073, 2011. 
[37] L. Hyunsoo, L. Naesung, S. Yongho, E. Jonghwa and L. S. Wook, "Comparison of frictional forces on graphene and graphite," Nanotechnology, vol. 20, 2009.

[38] Z. Deng, A. Smolyanitsky, Q. Li, X.-Q. Feng and R. J. Cannara, "Adhesion-dependent negative friction coefficient on chemically modified graphite at the nanoscale," Nature materials, vol. 11, p. 1032-1037, 2012.

[39] Q. Li, C. Lee, R. W. Carpick and J. Hone, "Substrate effect on thickness-dependent friction on graphene," Physica Status Solidi, vol. 247, pp. 2909- 2914, 2010.

[40] K. Jae-Hyeon, K. Sangku, B. Ik-Su, C. J. Sik, P. B. Ho, K. Yong-Hyun and P. J. Young, "Nanotribological Properties of Fluorinated, Hydrogenated, and Oxidized Graphenes," Tribology Letters, vol. 50, pp. 137-144, 2013.

[41] F.Bonelli, N. Manini, E. Cadelano and L. Colombo, "Atomistic Simulations of the Sliding Friction of Graphene Flakes," The European Physical Journal B, vol. 70, pp. 449-459, 2009.

[42] Y. Eswaraiah, V. Sankaranarayanan and a. S. Ramaprabhu, "Graphene based engine oil nanofluids for tribological applications," ACS Applied Materials and Interfaces, vol. 3, p. 4221-4227, 2011.

[43] W. Zhang, M. Zhou, H. Zhu, Y. Tian, K. Wang, J. Wei and F. Ji, "Tribological properties of oleic acid-modified graphene as lubricant oil additives," Journal of Physics D: Applied Physics, vol. 44, 2011. 
[44] K. S. Novoselov, D. Jiang1, T. Booth, V. Khotkevich, S. M. Morozov and A. K. Geim, "Twodimensional atomic crystals," Proceedings of the National Academy of Sciences of the United States of America, vol. 102, 2005.

[45] P. Blake, E. W. Hill, A. H. C. Neto, K. S. Novoselov, D. Jiang, R. Yang, T. J. Booth and A. K. Geim, "Making graphene visible," Applied Physics Letters, vol. 91, 2007.

[46] D. Wencheng, J. Xiaoqing and Z. Lihua, "From graphite to graphene: direct liquid-phase exfoliation of graphite to produce single and few layered pristine graphene," Journal of Materials Chemistry A, p. 10592-10606, 2013.

[47] Y. Hernandez, V. Nicolosi, M. Lotya, F. M. Blighe, Z. Sun, S. De, I. T. McGovern, B. Holland, M. Byrne, Y. K. Gun'Ko, J. J. Boland, P. Niraj, G. Duesberg and S. Krishnamurthy, "High-yield production of graphene by liquid-phase exfoliation of graphite," Nature Nanotechnology, no. 3, 2008.

[48] S. Raghavan, T. J. Denig, T. C. Nelson and C. D. Stinespring, "Novel surface chemical synthesis route for large area graphene-on-insulator films," Journal of Vacuum Science \& Technology B, vol. 30, 2011.

[49] G. Binnig, C. F. Quate and C. Gerber, "Atomic Force Microscope," Physical Review Letters, vol. 56, 1986.

[50] P. Nemes-Incze, Z. Osváth, K. Kamarás and L. Biró, "Anomalies in thickness measurements of graphene and few layer graphite crystals by tapping mode atomic force microscopy," Carbon, vol. 46, pp. 1435-1442, 2008. 
[51] V. Shahin, Y. Ludwig, C. Schafer, D. Nikova and H. Oberleithner, "Glucocorticoids remodel nuclear envelope structure and permeability," Journal of Cell Science, vol. 118, pp. 28812889, 2005.

[52] W. Johnson, "Using Thermal K to Calibrate the Spring Constants (k) of AFM Probes," 05 December 2008. [Online]. Available: http://www.toyo.co.jp/file/pdf/spm/files/agi/59903293EN.pdf. [Accessed 09 February 2015].

[53] C. P. Green, H. Lioe, J. P. Cleveland, R. Proksch, P. Mulvaney and J. E. Sader, "Normal and torsional spring constants of atomic force microscope cantilevers," Review of Scientific Instruments, vol. 75, 2004.

[54] K.-S. Kim, H.-J. Lee, C. Lee, S.-K. Lee, H. Jang, J.-H. Ahn, J.-H. Kim and H.-J. Lee, "Chemical Vapor Deposition-Grown Graphene: The Thinnest Solid Lubricant," ACS Nano, vol. 5, pp. 5107-5114, 2011.

[55] H.-Y. Nie, "Scanning Probe Techniques," [Online]. Available: http://publish.uwo.ca/ hnie/spmman.html. [Accessed 30 April 2015].

[56] S. Stankovich, D. A. Dikin, R. D. Piner, K. A. Kohlhaas, A. Kleinhammes, Y. Jia, Y. Wu, S. T. Nguyen and R. S. Ruoff, "Synthesis of graphene-based nanosheets via chemical reduction of exfoliated graphite oxide," Carbon, vol. 45, pp. 1558-1565, 2007.

[57] D. Yang, A. Velamakanni, G. Bozoklu, S. Park, M. Stoller, R. D. Piner, S. Stankovich, I. Jung, D. A. Field, C. A. V. Jr. and R. S. Ruoff, "Chemical analysis of graphene oxide films 
after heat and chemical treatments by X-ray photoelectron and Micro-Raman spectroscopy," Carbon, vol. 47, pp. 145-152, 2009.

[58] C. H. Lui, L. Liu, K. F. Mak, G. W. Flynn and T. F. Heinz, "Ultraflat graphene," Nature, vol. 462, pp. 339-341, 2009.

[59] T. Li and Z. Zhang, "Snap-through instability of graphene on substrates," Nanoscale Research Letters, vol. 5, pp. 169-173, 2010.

[60] H.Pourzand, "Thickness dependent adhesion force and its correlation to surface roughness in multilayered graphene," in SENSORS, 2013 IEEE, Baltimore, 2013.

[61] Z. Deng, N. N. Klimov, S. D. Solares, T. Li and H. Xu, "Nanoscale Interfacial Friction and Adhesion on Supported versus Suspended Monolayer and Multilayer Graphene," Langmuir, vol. 29, pp. 235-243, 2013.

[62] T. Eastman and D.-M. Zhu, "Adhesion Forces between Surface-Modified AFM Tips and a Mica Surface," Langmuir, vol. 12, pp. 2859- 2862, 1996.

[63] D. Yalin, "Effects of substrate roughness and electron-phonon coupling on thicknessdependent friction of graphene," Journal of Physics D: Applied Physics, vol. 47, 2014.

[64] L. Sirghi, "Effect of capillary-condensed water on the dynamic friction force at nanoasperity contacts," Applied Physics Letters, vol. 82, 2003.

[65] A. Labuda and P. Grütter, "Atomic Force Microscopy in Viscous Ionic Liquids," Langmuir, vol. 28, pp. 5319 - 5322, 2012. 
[66] S. Dorbritz, W. Ruth and U. Krag, "Investigation on Aggregate Formation of Ionic Liquids," Advanced Synthesis and Catalysis, vol. 347, pp. 1273-1279, 2005.

[67] B. Bhushan, M. Palacio and B. Kinzig, "AFM-based nantribological and electrical characterization of ultathin wear-resistant ionic liquid films," Journal of Colloid and Interface Science, vol. 317, pp. 275-287, 2007.

[68] J. M. Coles, D. P. Chang and S. Zauscher, "Current Opinion in Colloid \& Interface Science," Science Direct, vol. 15, pp. 406-416, 2010.

[69] K. Yuriko, K. Tahahiro and S. Shinya, "Tribological Properties of Ionic Liquids, Ionic Liquids - New Aspects for the Future," Intech, 2013. 